\title{
MAXIMAL SUBSEMIGROUPS OF THE SEMIGROUP OF ALL MAPPINGS ON AN INFINITE SET
}

\author{
J. EAST, J. D. MITCHELL, AND Y. PÉRESSE
}

\begin{abstract}
In this paper we classify the maximal subsemigroups of the full transformation semigroup $\Omega^{\Omega}$, which consists of all mappings on the infinite set $\Omega$, containing certain subgroups of the symmetric group $\operatorname{Sym}(\Omega)$ on $\Omega$. In 1965 Gavrilov showed that there are five maximal subsemigroups of $\Omega^{\Omega}$ containing $\operatorname{Sym}(\Omega)$ when $\Omega$ is countable, and in 2005 Pinsker extended Gavrilov's result to sets of arbitrary cardinality.

We classify the maximal subsemigroups of $\Omega^{\Omega}$ on a set $\Omega$ of arbitrary infinite cardinality containing one of the following subgroups of $\operatorname{Sym}(\Omega)$ : the pointwise stabiliser of a non-empty finite subset of $\Omega$, the stabiliser of an ultrafilter on $\Omega$, or the stabiliser of a partition of $\Omega$ into finitely many subsets of equal cardinality. If $G$ is any of these subgroups, then we deduce a characterisation of the mappings $f, g \in \Omega^{\Omega}$ such that the semigroup generated by $G \cup\{f, g\}$ equals $\Omega^{\Omega}$.
\end{abstract}

\section{INTRODUCTION}

A subgroup $H$ of a group $G$ is a maximal subgroup if $H \neq G$ and the subgroup generated by $H$ and $g$ equals $G$ for all $g \in G \backslash H$. The definition of a maximal subsemigroup of a semigroup is analogous: a subsemigroup $T$ of a semigroup (or group) $S$ is a maximal subsemigroup if $T \neq S$ and the subsemigroup $\langle T, s\rangle$ generated by $T$ and $s$ equals $S$ for all $s \in S \backslash T$.

Let $\Omega$ denote an arbitrary (finite or infinite) set, let $\Omega^{\Omega}$ denote the semigroup of mappings from $\Omega$ to itself, and let $\operatorname{Sym}(\Omega)$ denote the symmetric group on $\Omega$. In this paper we are interested in those maximal subsemigroups of $\Omega^{\Omega}$ that contain certain subgroups of $\operatorname{Sym}(\Omega)$. The maximal subgroups of finite symmetric groups, having been investigated by O'Nan and Scott [28, Aschbacher and Scott [1], and Liebeck, Praeger and Saxl [18], are, in some sense, known. When $\Omega$ is finite, it is easy to see that a maximal subsemigroup of $\Omega^{\Omega}$ is either the union of a maximal subgroup of the symmetric group and $\Omega^{\Omega} \backslash \operatorname{Sym}(\Omega)$ or it is the union of $\operatorname{Sym}(\Omega)$ and the mappings with at most $|\Omega|-2$ points in their images. In general, the maximal subsemigroups of an arbitrary finite semigroup are determined, roughly speaking, by their maximal subgroups; see Graham, Graham, and Rhodes [12].

Maximal subgroups of $\operatorname{Sym}(\Omega)$ have also been extensively studied when $\Omega$ is infinite; see $[2,4,6,8,19,20,22,27]$ and the references therein. It seems extremely unlikely that a complete description, in any sense, of maximal subgroups of $\operatorname{Sym}(\Omega)$ exists for infinite $\Omega$. Maximal subsemigroups of $\Omega^{\Omega}$ when $\Omega$ is infinite have been considered to a lesser degree. The maximal subsemigroups of $\Omega^{\Omega}$ containing the symmetric group were classified by Gavrilov in [11] for countable $\Omega$ and by Pinsker

Received by the editors September 18, 2012 and, in revised form, February 11, 2013. 2010 Mathematics Subject Classification. Primary 20B30, 20B35, 20M20. 
[24, Theorem 1.4] for arbitrary infinite $\Omega$; these are the only results regarding maximal subsemigroups of $\Omega^{\Omega}$ when $\Omega$ is infinite of which we are aware. We state and prove Gavrilov and Pinsker's theorem (Theorem $\mathrm{A}$ ) since elements of the proof are required later on, for the sake of completeness, and for the convenience of the reader. Maximal subsemigroups of other infinite semigroups of mappings have been considered. For example, Levi and Wood [17] and Hotzel [13 considered maximal subsemigroups of Baer-Levi semigroups, and Shneperman 29 considered the maximal subsemigroups of the endomorphism monoid of a finite dimensional complex vector space that are maximal with respect to being compact.

The subsemigroups of $\Omega^{\Omega}$ form an algebraic lattice with $2^{|\Omega|}$ compact elements under inclusion. The study of maximal subsemigroups of $\Omega^{\Omega}$ belongs to the wider study of this lattice. Pinsker and Shelah [26] prove that every algebraic lattice with at most $2^{|\Omega|}$ compact elements can be embedded into the subsemigroup lattice of $\Omega^{\Omega}$. There are $2^{2^{|\Omega|}}$ distinct subsemigroups of $\Omega^{\Omega}$. There are even $2^{2^{\kappa}}$ subsemigroups between $\operatorname{Sym}(\Omega)$ and any maximal subsemigroup of $\Omega^{\Omega}$ that contains $\operatorname{Sym}(\Omega)$ where $|\Omega|=\aleph_{\alpha}$ and $\kappa=\max \left\{\alpha, \aleph_{0}\right\}$; for further details see Pinsker [25]. We show, as a consequence of Theorem $\left[\right.$ that there are also $2^{2^{|\Omega|}}$ maximal subsemigroups of $\Omega^{\Omega}$. The maximal subsemigroups of the maximal subsemigroups described by Gavrilov [11] are classified in [10]; perhaps surprisingly there are only countably many such semigroups. In further contrast to Pinsker and Shelah's result [26], there are only 38 subsemigroups between the intersection $S_{1} \cap S_{2} \cap S_{3}\left(\aleph_{0}\right) \cap S_{4}\left(\aleph_{0}\right) \cap S_{5}$ of the maximal subsemigroups described by Gavrilov [11] and $\Omega^{\Omega}$; see Mitchell and Jonušas [16].

Another natural question to ask about the subsemigroup lattice of $\Omega^{\Omega}$ is whether or not every subsemigroup is contained in a maximal one. In 4] it is shown that under certain set theoretic assumptions there exists a $\operatorname{subgroup~of~} \operatorname{Sym}(\Omega)$ that is not contained in a maximal subgroup; it seems likely that the analogous result holds for $\Omega^{\Omega}$. There are several results in the literature concerning sufficient conditions for a subgroup of $\operatorname{Sym}(\Omega)$ to lie in a maximal subgroup; see [20] and [21]. In Section 3 we explore the analogous problem for subsemigroups of $\Omega^{\Omega}$.

In this paper we classify the maximal subsemigroups of $\Omega^{\Omega}$, where $\Omega$ is any infinite set, containing certain subgroups of $\operatorname{Sym}(\Omega)$, which we define in the next section. In particular, we classify the maximal subsemigroups of $\Omega^{\Omega}$ containing one of the following groups: the symmetric group $\operatorname{Sym}(\Omega)$ (Theorem $\underline{\mathrm{A}}$ ), the pointwise stabiliser of a non-empty finite subset of $\Omega$ (Theorem $\bar{B}$ ), the stabiliser of an ultrafilter on $\Omega$ (Theorem $[\mathrm{C}$ ), or the stabiliser of a finite partition of $\Omega$ (Theorem $\mathrm{D}$ ). For each of these subgroups, we obtain a characterisation of those pairs of elements that, together with the subgroup, generate $\Omega^{\Omega}$; see Corollaries 4.2, 4.3, 4.4 and 4.5 . Such a classification in the case that $G=\operatorname{Sym}(\Omega)$ and $|\Omega|$ is a regular cardinal was originally given in [14, Theorem 3.3]. As previously mentioned, the classification of maximal subsemigroup of $\Omega^{\Omega}$ containing $\operatorname{Sym}(\Omega)$ is originally due to Gavrilov 11 . and Pinsker 24.

The paper is organised as follows: in Section 2, we state the main theorems of the paper. In Section 3 we give several sufficient conditions for a subsemigroup of $\Omega^{\Omega}$ to be contained in a maximal subsemigroup, and also give a new proof of the result of Macpherson and Praeger 21] which states that every subgroup of $\operatorname{Sym}(\Omega)$ that is not highly transitive is contained in a maximal subgroup. In Section 4, we state and prove Corollaries 4.2, 4.3, 4.4 and 4.5. In Section 5. we give several 
technical results which underpin the proofs of the main results in the paper. In Sections 6, 7, 8, and 9, we give the respective proofs of the four main theorems from Section 2. In Section 10, we show that the setwise stabiliser of a non-empty finite set, the almost stabiliser of a finite partition, and the stabiliser of an ultrafilter are maximal subsemigroups (and not just maximal subgroups as is already wellknown) of the symmetric group.

We end this section by asking the three most interesting questions, in our eyes at least, arising from our consideration of maximal subsemigroups of $\Omega^{\Omega}$.

Question 1.1. Let $G$ be a maximal subsemigroup of $\operatorname{Sym}(\Omega)$. Then does there exist a maximal subsemigroup $M$ of $\Omega^{\Omega}$ such that $M \cap \operatorname{Sym}(\Omega)=G$ ?

The intersection of every known example of a maximal subsemigroup of $\Omega^{\Omega}$ with $\operatorname{Sym}(\Omega)$ is either a maximal subsemigroup of $\operatorname{Sym}(\Omega)$ or $\operatorname{Sym}(\Omega)$ itself, which prompts the following question.

Question 1.2. Does there exist a maximal subsemigroup of $\Omega^{\Omega}$ that does not contain a maximal subsemigroup of $\operatorname{Sym}(\Omega)$ ?

We suspect that the answer to Question 1.2 is yes. A step in the other direction would, perhaps, be a positive answer to the following question.

Question 1.3. Does every maximal subsemigroup of $\Omega^{\Omega}$ have non-trivial intersection with $\operatorname{Sym}(\Omega)$ ?

\section{Statements of the main theorems}

Throughout the paper we write functions to the right of their argument and compose from left to right. If $\alpha \in \Omega, f \in \Omega^{\Omega}$ and $\Sigma \subseteq \Omega$, then $\alpha f^{-1}=\{\beta \in \Omega$ : $\beta f=\alpha\}, \Sigma f=\{\alpha f: \alpha \in \Sigma\}$, and $\left.f\right|_{\Sigma}$ denotes the restriction of $f$ to $\Sigma$. We denote $\left\{f \in \Omega^{\Omega}:|\Omega f|<|\Omega|\right\}$ by $\mathfrak{F}$. Since $\mathfrak{F}$ is an ideal of $\Omega^{\Omega}$, if $S$ is any subsemigroup of $\Omega^{\Omega}$, then so is $S \cup \mathfrak{F}$. Hence if $S$ is maximal, then either $\mathfrak{F} \subseteq S$ or $S \cup \mathfrak{F}=\Omega^{\Omega}$. In the latter case, $\Omega^{\Omega} \backslash \mathfrak{F}$ is a subset of $S$. But $\Omega^{\Omega} \backslash \mathfrak{F}$ is also a generating set for $\Omega^{\Omega}$ and so $S=\Omega^{\Omega}$, which contradicts the assumption that $S$ is a maximal subsemigroup. Hence $\mathfrak{F}$ is contained in every maximal subsemigroup of $\Omega^{\Omega}$.

Let $\Sigma$ be any subset of $\Omega$ and let $f: \Sigma \rightarrow \Omega$ be arbitrary. If $\Gamma \subseteq \Sigma$ such that $\left.f\right|_{\Gamma}$ is injective and $\Gamma f=\Sigma f$, then we will refer to $\Gamma$ as a transversal of $f$. We require the following parameters of $f$ to state our main theorems:

$$
\begin{aligned}
d(f) & =|\Omega \backslash \Sigma f| \\
c(f) & =|\Sigma \backslash \Gamma|, \text { where } \Gamma \text { is any transversal of } f \\
k(f, \mu) & =\left|\left\{\alpha \in \Omega:\left|\alpha f^{-1}\right| \geq \mu\right\}\right|, \text { where } \mu \leq|\Omega| .
\end{aligned}
$$

The parameters $d(f), c(f)$, and $k(f,|\Omega|)$ were termed the defect, collapse, and infinite contraction index, respectively, of $f$ in 14 .

As usual, we will think of a cardinal $\kappa$ as the set of all ordinals strictly less than $\kappa$. Recall that a cardinal $\kappa$ is singular if there exists a cardinal $\lambda<\kappa$ and a family of sets $\Sigma_{\mu}(\mu \in \lambda)$ such that $\left|\Sigma_{\mu}\right|<\kappa$ for each $\mu<\lambda$, yet $\left|\bigcup_{\mu<\lambda} \Sigma_{\mu}\right|=\kappa$; otherwise, $\kappa$ is regular. We denote the successor to any cardinal $\kappa$ by $\kappa^{+}$.

A subset $\Sigma$ of an infinite set $\Gamma$ is a moiety of $\Gamma$ if $|\Sigma|=|\Gamma \backslash \Sigma|=|\Gamma|$. 


\subsection{The symmetric group.}

Theorem A (Gavrilov [11, Pinsker [24]). Let $\Omega$ be any infinite set. If $|\Omega|$ is a regular cardinal, then the maximal subsemigroups of $\Omega^{\Omega}$ containing $\operatorname{Sym}(\Omega)$ are:

$$
\begin{aligned}
S_{1} & =\left\{f \in \Omega^{\Omega}: c(f)=0 \text { or } d(f)>0\right\}, \\
S_{2} & =\left\{f \in \Omega^{\Omega}: c(f)>0 \text { or } d(f)=0\right\}, \\
S_{3}(\mu) & =\left\{f \in \Omega^{\Omega}: c(f)<\mu \text { or } d(f) \geq \mu\right\}, \\
S_{4}(\mu) & =\left\{f \in \Omega^{\Omega}: c(f) \geq \mu \text { or } d(f)<\mu\right\}, \\
S_{5} & =\left\{f \in \Omega^{\Omega}: k(f,|\Omega|)<|\Omega|\right\},
\end{aligned}
$$

where $\mu$ is any infinite cardinal not greater than $|\Omega|$.

If $|\Omega|$ is a singular cardinal, then the maximal subsemigroups of $\Omega^{\Omega}$ containing $\operatorname{Sym}(\Omega)$ are $S_{1}, S_{2}, S_{3}(\mu), S_{4}(\mu)$, where $\mu$ is any infinite cardinal not greater than $|\Omega|$, and

$$
S_{5}^{\prime}=\left\{f \in \Omega^{\Omega}:(\exists \nu<|\Omega|)(k(f, \nu)<|\Omega|)\right\} .
$$

The countable case of Theorem $\mathrm{A}$ was first proved by Gavrilov [11. The full version of Theorem A given above was first proved by Pinsker [24, Theorem 1.4]. We independently proved Theorem $\mathrm{A}$ whilst unaware of the work of Gavrilov and Pinsker. We thank Martin Goldstern and Lutz Heindorf for bringing these references to our attention. A full proof of Theorem $\mathrm{A}$ is included in Section 6 for the convenience of the reader and for the sake of completeness.

2.2. The pointwise stabiliser of a finite set. If $G$ is a group acting on a set $\Omega$ and $\Sigma$ is any subset of $\Omega$, then we denote the pointwise stabiliser of $\Sigma$ under $G$ by $G_{(\Sigma)}$ and the setwise stabiliser of $\Sigma$ under $G$ by $G_{\{\Sigma\}}$. In [2], it is shown that if $\Sigma$ is a non-empty finite subset of $\Omega$, then $\operatorname{Sym}(\Omega)_{\{\Sigma\}}$ is a maximal subgroup of $\operatorname{Sym}(\Omega)$.

Theorem B. Let $\Omega$ be any infinite set and let $\Sigma$ be a non-empty finite subset of $\Omega$. Then the maximal subsemigroups of $\Omega^{\Omega}$ containing the pointwise stabiliser $\operatorname{Sym}(\Omega)_{(\Sigma)}$ but not $\operatorname{Sym}(\Omega)$ are:

$$
\begin{aligned}
& F_{1}(\Gamma, \mu)=\left\{f \in \Omega^{\Omega}: d(f) \geq \mu \text { or } \Gamma \nsubseteq \Omega f \text { or }\left(\Gamma f^{-1} \subseteq \Gamma \text { and } c(f)<\mu\right)\right\} \cup \mathfrak{F}, \\
& F_{2}(\Gamma, \nu)=\left\{f \in \Omega^{\Omega}: c(f) \geq \nu \text { or }|\Gamma f|<|\Gamma| \text { or }(\Gamma f=\Gamma \text { and } d(f)<\nu)\right\} \cup \mathfrak{F},
\end{aligned}
$$

where $\Gamma$ is a non-empty subset of $\Sigma$ and $\mu$ and $\nu$ are infinite cardinals with $\mu \leq|\Omega|^{+}$ and either $|\Gamma|=1$ and $\nu=|\Omega|^{+}$or $|\Gamma| \geq 2$ and $\nu \leq|\Omega|^{+}$.

If $\mu \leq|\Omega|$ and $f \in \mathfrak{F}$, then $d(f)=|\Omega|=c(f)$, and so " $\cup \mathfrak{F}$ " could be omitted from the definition of $F_{1}(\Gamma, \mu)$ and $F_{2}(\Gamma, \mu)$ in these cases.

If $|\Gamma|=1$, then $F_{2}(\Gamma, \nu)$ is properly contained in $S_{4}(\nu)$ for all $\nu \leq|\Omega|$. In particular, $F_{2}(\Gamma, \nu)$ is not maximal in this case. When $\mu$ or $\nu$ equals $|\Omega|^{+}$, we obtain the following simpler definitions of the semigroups in Theorem B;

$$
\begin{aligned}
& F_{1}\left(\Gamma,|\Omega|^{+}\right)=\left\{f \in \Omega^{\Omega}: \Gamma \nsubseteq \Omega f \text { or } \Gamma f^{-1} \subseteq \Gamma\right\} \cup \mathfrak{F}, \\
& F_{2}\left(\Gamma,|\Omega|^{+}\right)=\left\{f \in \Omega^{\Omega}:|\Gamma f|<|\Gamma| \text { or } \Gamma f=\Gamma\right\} \cup \mathfrak{F} .
\end{aligned}
$$


In particular, if $\Gamma=\{\gamma\}$, then

$$
\begin{aligned}
& F_{1}\left(\Gamma,|\Omega|^{+}\right)=\left\{f \in \Omega^{\Omega}: \gamma \notin \Omega f \text { or } \gamma f^{-1}=\{\gamma\}\right\} \cup \mathfrak{F}, \\
& F_{2}\left(\Gamma,|\Omega|^{+}\right)=\left\{f \in \Omega^{\Omega}: \gamma f=\gamma\right\} \cup \mathfrak{F} .
\end{aligned}
$$

If $\Gamma$ is any finite subset of $\Omega$, then the intersection of $F_{1}(\Gamma, \mu)$ or $F_{2}(\Gamma, \mu)$ with $\operatorname{Sym}(\Omega)$ is the setwise stabiliser $\operatorname{Sym}(\Omega)_{\{\Gamma\}}$. Thus every maximal subsemigroup of $\Omega^{\Omega}$ containing the pointwise stabiliser of a finite subset $\Sigma$ of $\Omega$ also contains the setwise stabiliser of some subset $\Gamma$ of $\Sigma$. Since $\operatorname{Sym}(\Omega)_{\{\Sigma\}}$ is a maximal subgroup of $\operatorname{Sym}(\Omega)$, it follows that the maximal subsemigroups of $\Omega^{\Omega}$ containing $\operatorname{Sym}(\Omega)_{\{\Sigma\}}$ but not $\operatorname{Sym}(\Omega)$ are those listed in Theorem $\mathrm{B}$ where $\Gamma=\Sigma$.

2.3. The stabiliser of an ultrafilter. A set of subsets $\mathcal{F}$ of $\Omega$ is called a filter if:

(i) $\emptyset \notin \mathcal{F}$;

(ii) if $\Sigma \in \mathcal{F}$ and $\Sigma \subseteq \Gamma \subseteq \Omega$, then $\Gamma \in \mathcal{F}$;

(iii) if $\Sigma, \Gamma \in \mathcal{F}$, then $\Sigma \cap \Gamma \in \mathcal{F}$.

A filter is called an ultrafilter if it is maximal with respect to containment among filters on $\Omega$. Equivalently, a filter $\mathcal{F}$ is an ultrafilter if, for every $\Sigma \subseteq \Omega$, either $\Sigma \in \mathcal{F}$ or $\Omega \backslash \Sigma \in \mathcal{F}$. An ultrafilter $\mathcal{F}$ on $\Omega$ is principal if there exists $\alpha \in \Omega$ such that $\mathcal{F}=\{\Sigma \subseteq \Omega: \alpha \in \Sigma\}$. An ultrafilter $\mathcal{F}$ is uniform if $|\Sigma|=|\Omega|$ for all $\Sigma \in \mathcal{F}$. The stabiliser of a filter $\mathcal{F}$ in $\operatorname{Sym}(\Omega)$ is defined to be

$$
\{f \in \operatorname{Sym}(\Omega):(\forall \Sigma \subseteq \Omega)(\Sigma \in \mathcal{F} \leftrightarrow \Sigma f \in \mathcal{F})\}
$$

and is denoted by $\operatorname{Sym}(\Omega)_{\{\mathcal{F}\}}$. The stabiliser of an ultrafilter is the union of the pointwise stabilisers of the sets in the filter, i.e.

$$
\operatorname{Sym}(\Omega)_{\{\mathcal{F}\}}=\bigcup_{\Sigma \in \mathcal{F}} \operatorname{Sym}(\Omega)_{(\Sigma)}
$$

see [20, Theorem 6.4]. It is shown in [20, Theorem 6.4] and [27] that the stabiliser $\operatorname{Sym}(\Omega)_{\{\mathcal{F}\}}$ of any ultrafilter is a maximal subgroup of the symmetric group.

Let $\mathcal{F}$ be any filter on $\Omega$ and let $\mu$ be an infinite cardinal. Then we define the following subsemigroups of $\Omega^{\Omega}$ :

$$
\begin{aligned}
& U_{1}(\mathcal{F}, \mu)=\left\{f \in \Omega^{\Omega}:(d(f) \geq \mu) \text { or }(\Omega f \notin \mathcal{F})\right. \\
& \text { or }(c(f)<\mu \text { and }(\forall \Sigma \notin \mathcal{F})(\Sigma f \notin \mathcal{F}))\} \cup \mathfrak{F} ; \\
& U_{2}(\mathcal{F}, \mu)=\left\{f \in \Omega^{\Omega}:(c(f) \geq \mu) \text { or }(\forall \Sigma \in \mathcal{F})\left(c\left(\left.f\right|_{\Sigma}\right)>0\right)\right. \\
& \text { or }(d(f)<\mu \text { and }(\forall \Sigma \in \mathcal{F})(\Sigma f \in \mathcal{F}))\} \cup \mathfrak{F} .
\end{aligned}
$$

If $\mu \leq|\Omega|$ and $f \in \mathfrak{F}$, then $d(f)=|\Omega|=c(f)$, and so " $\cup \mathfrak{F}$ " could be omitted from the definition of $U_{1}(\mathcal{F}, \mu)$ and $U_{2}(\mathcal{F}, \mu)$ in these cases.

If $\Gamma$ is any subset of $\Omega$, then the collection $\mathcal{F}$ of subsets of $\Omega$ containing $\Gamma$ is a filter. In this case, the stabiliser of $\mathcal{F}$ in $\operatorname{Sym}(\Omega)$ and the setwise stabiliser of $\Gamma$ in $\operatorname{Sym}(\Omega)$ coincide. In the following lemma, we show that $U_{1}(\mathcal{F}, \mu)$ and $U_{2}(\mathcal{F}, \mu)$ are the generalisations of the semigroups in Theorem $\mathrm{B}$ to arbitrary filters.

Lemma 2.1. Let $\Gamma$ be a non-empty finite subset of $\Omega$ and let $\mathcal{F}$ be the filter consisting of subsets of $\Omega$ containing $\Gamma$. Then $F_{1}(\Gamma, \mu)=U_{1}(\mathcal{F}, \mu)$ and $F_{2}(\Gamma, \mu)=$ $U_{2}(\mathcal{F}, \mu)$ for all infinite cardinals $\mu$. 
Proof. Suppose that $f \in \Omega^{\Omega}$. Clearly, $\Omega f \notin \mathcal{F}$ if and only if $\Gamma \nsubseteq \Omega \Omega f$. Also $\Sigma f \notin \mathcal{F}$ for all $\Sigma \notin \mathcal{F}$ if and only if $\Gamma \nsubseteq \Sigma f$ for all $\Sigma \subseteq \Omega$ such that $\Gamma \nsubseteq \Sigma$ if and only if $\Gamma f^{-1} \subseteq \Gamma$. Therefore $F_{1}(\Gamma, \mu)=U_{1}(\mathcal{F}, \mu)$.

It is straightforward to show that $c\left(\left.f\right|_{\Sigma}\right)>0$ for all $\Sigma \in \mathcal{F}$ if and only if no transversal of $f$ belongs to $\mathcal{F}$ if and only if $\Gamma$ is not a subset of any transversal of $f$ if and only if $|\Gamma f|<|\Gamma|$. Suppose that $|\Gamma f|=|\Gamma|$. Then $\Sigma f \in \mathcal{F}$ for all $\Sigma \in \mathcal{F}$ if and only if $\Gamma \subseteq \Sigma f$ for all $\Sigma \subseteq \Omega$ such that $\Gamma \subseteq \Sigma$ if and only if $\Gamma f \subseteq \Gamma$ if and only if $\Gamma f=\Gamma$. Thus $F_{2}(\Gamma, \nu)=U_{2}(\mathcal{F}, \nu)$, as required.

The semigroups in Theorem B contain not only the pointwise stabiliser, but the setwise stabiliser of a finite set. It follows that the maximal subsemigroups of $\Omega^{\Omega}$ containing the stabiliser of a filter generated by a finite set, in particular principal ultrafilters, have already been classified in Theorem B. For the sake of convenience, we state the analogue of Theorem $\mathrm{B}$ in terms of filters.

Corollary 2.2. Let $\Omega$ be any infinite set, let $\Gamma$ be a non-empty finite subset of $\Omega$, and let $\mathcal{F}$ be the filter consisting of subsets of $\Omega$ containing $\Gamma$. Then the maximal subsemigroups of $\Omega^{\Omega}$ containing $\operatorname{Sym}(\Omega)_{\{\mathcal{F}\}}$ but not $\operatorname{Sym}(\Omega)$ are $F_{1}(\Gamma, \mu)=$ $U_{1}(\mathcal{F}, \mu)$ and $F_{2}(\Gamma, \nu)=U_{2}(\mathcal{F}, \nu)$, where $\mu$ and $\nu$ are infinite cardinals with $\mu \leq$ $|\Omega|^{+}$and either $|\Gamma|=1$ and $\nu=|\Omega|^{+}$or $|\Gamma| \geq 2$ and $\nu \leq|\Omega|^{+}$.

If $|\Gamma|=1$, then $\mathcal{F}$ in Corollary 2.2 is a principal ultrafilter. Replacing this principal ultrafilter by a non-principal ultrafilter yields the following theorem, which is similar to Corollary 2.2 the main difference being the possible values that the cardinals $\mu$ and $\nu$ can have.

Theorem C. Let $\Omega$ be any infinite set, let $\mathcal{F}$ be a non-principal ultrafilter on $\Omega$, and let $\kappa\left(\geq \aleph_{0}\right)$ be the least cardinality of a subset of $\Omega$ in $\mathcal{F}$. Then the maximal subsemigroups of $\Omega^{\Omega}$ containing $\operatorname{Sym}(\Omega)_{\{\mathcal{F}\}}$ but not $\operatorname{Sym}(\Omega)$ are $U_{1}(\mathcal{F}, \mu)$ and $U_{2}(\mathcal{F}, \mu)$ where $\mu$ is an infinite cardinal such that $\kappa<\mu \leq|\Omega|^{+}$.

Suppose that $\mathcal{F}$ is a non-principal ultrafilter. If $f \in \Omega^{\Omega}$ such that $\Omega f \notin \mathcal{F}$, then $\Omega \backslash \Omega f \in \mathcal{F}$ and so $d(f)=|\Omega \backslash \Omega f| \geq \kappa$. Hence if $\mu \leq \kappa$, then

$$
U_{1}(\mathcal{F}, \mu)=\left\{f \in \Omega^{\Omega}:(d(f) \geq \mu) \text { or }(c(f)<\mu \text { and }(\forall \Sigma \notin \mathcal{F})(\Sigma f \notin \mathcal{F}))\right\} \subsetneq S_{3}(\mu),
$$

and $U_{1}(\mathcal{F}, \mu)$ is not maximal in this case. If $f \in \Omega^{\Omega}$ is such that $c\left(\left.f\right|_{\Sigma}\right)>0$ for all $\Sigma \in \mathcal{F}$, then no transversal of $f$ belongs to $\mathcal{F}$. Hence the complement of any transversal of $f$ belongs to $\mathcal{F}$, and so $c(f) \geq \kappa$. In particular, if $\mu \leq \kappa$, then

$U_{2}(\mathcal{F}, \mu)=\left\{f \in \Omega^{\Omega}:(c(f) \geq \mu)\right.$ or $(d(f)<\mu$ and $\left.\left.(\forall \Sigma \in \mathcal{F})(\Sigma f \in \mathcal{F}))\right)\right\} \subsetneq S_{4}(\mu)$, and so $U_{2}(\mathcal{F}, \mu)$ is also not maximal in this case.

If $\mathcal{F}$ in Theorem $\mathbb{C}$ is a uniform ultrafilter, then $\kappa=|\Omega|$ and so there is only one possible value for $\mu$, namely $|\Omega|^{+}$, and the conditions on $U_{1}\left(\mathcal{F},|\Omega|^{+}\right)$and $U_{2}\left(\mathcal{F},|\Omega|^{+}\right)$become much simpler:

$$
\begin{aligned}
& U_{1}\left(\mathcal{F},|\Omega|^{+}\right)=\left\{f \in \Omega^{\Omega}:(\forall \Sigma \notin \mathcal{F})(\Sigma f \notin \mathcal{F})\right\} \cup \mathfrak{F}, \\
& U_{2}\left(\mathcal{F},|\Omega|^{+}\right)=\left\{f \in \Omega^{\Omega}:(\forall \Sigma \in \mathcal{F})\left(c\left(\left.f\right|_{\Sigma}\right)>0\right) \text { or }(\forall \Sigma \in \mathcal{F})(\Sigma f \in \mathcal{F})\right\} \cup \mathfrak{F} .
\end{aligned}
$$

There are $2^{|\Omega|}$ elements in $\operatorname{Sym}(\Omega)$, and by Pospísil's Theorem [15, Theorem 7.6] there are $2^{2^{|\Omega|}}$ ultrafilters on $\Omega$. Hence there are $2^{2^{|\Omega|}}$ non-conjugate maximal subsemigroups of $\Omega^{\Omega}$. 
While the semigroups in Corollary 2.2 and Theorem $C$ have the same definitions in terms of their respective filters, neither result appears to be a corollary of the other. We were unable to formulate a more general theorem having Corollary 2.2 and Theorem C] let alone Theorem $\mathrm{B}$, as special cases.

2.4. The stabiliser of a finite partition. Let $n \geq 2$ and let $\mathcal{P}=\left\{\Sigma_{0}, \Sigma_{1}, \ldots\right.$, $\left.\Sigma_{n-1}\right\}$ be a partition of $\Omega$ such that $\left|\Sigma_{0}\right|=\cdots=\left|\Sigma_{n-1}\right|=|\Omega|$. We will refer to such a partition $\mathcal{P}$ as a finite partition of $\Omega$. The stabiliser of a finite partition $\mathcal{P}=\left\{\Sigma_{0}, \Sigma_{1}, \ldots, \Sigma_{n-1}\right\}$ is defined by

$$
\operatorname{Stab}(\mathcal{P})=\left\{f \in \operatorname{Sym}(\Omega):(\forall i)(\exists j)\left(\Sigma_{i} f=\Sigma_{j}\right)\right\},
$$

and the almost stabiliser of $\mathcal{P}$ is defined by

$$
\operatorname{AStab}(\mathcal{P})=\left\{f \in \operatorname{Sym}(\Omega):(\forall i)(\exists j)\left(\left|\Sigma_{i} f \backslash \Sigma_{j}\right|+\left|\Sigma_{j} \backslash \Sigma_{i} f\right|<|\Omega|\right)\right\} .
$$

Of course, $\operatorname{Stab}(\mathcal{P})$ is a subgroup of $\operatorname{AStab}(\mathcal{P})$ and $\operatorname{so} \operatorname{Stab}(\mathcal{P})$ is not a maximal subgroup of $\operatorname{Sym}(\Omega)$. On the other hand, it was shown in [27] (and [20] independently) that $\operatorname{AStab}(\mathcal{P})$ is a maximal subgroup of $\operatorname{Sym}(\Omega)$.

Let $f \in \Omega^{\Omega}$. Then define the binary relation $\rho_{f}$ on $\{0,1, \ldots, n-1\}$ by

$$
\rho_{f}=\left\{(i, j):\left|\Sigma_{i} f \cap \Sigma_{j}\right|=|\Omega|\right\} .
$$

If $\sigma$ is a binary relation on a set $\Omega$, then $\sigma^{-1}=\{(i, j):(j, i) \in \sigma\}$ and $\sigma$ is total if for all $\alpha \in \Omega$ there exists $\beta \in \Omega$ such that $(\alpha, \beta) \in \sigma$. We will write $\operatorname{Sym}(n)$ for the symmetric group on the set $n=\{0,1, \ldots, n-1\}$.

Theorem D. Let $\Omega$ be any infinite set and let $\mathcal{P}=\left\{\Sigma_{0}, \Sigma_{1}, \ldots, \Sigma_{n-1}\right\}, n \geq 2$, be a finite partition of $\Omega$. Then the maximal subsemigroups of $\Omega^{\Omega}$ containing $\operatorname{Stab}(\mathcal{P})$ but not $\operatorname{Sym}(\Omega)$ are:

$$
\begin{aligned}
& A_{1}(\mathcal{P})=\left\{f \in \Omega^{\Omega}: \rho_{f} \in \operatorname{Sym}(n) \text { or } \rho_{f} \text { is not total }\right\}, \\
& A_{2}(\mathcal{P})=\left\{f \in \Omega^{\Omega}: \rho_{f} \in \operatorname{Sym}(n) \text { or } \rho_{f}^{-1} \text { is not total }\right\} .
\end{aligned}
$$

If $\mathcal{P}$ is any finite partition of $\Omega$, then the intersection of $A_{1}(\mathcal{P})$ and $A_{2}(\mathcal{P})$ with $\operatorname{Sym}(\Omega)$ is the almost stabiliser $\operatorname{AStab}(\mathcal{P})$ of $\mathcal{P}$. Thus every maximal subsemigroup of $\Omega^{\Omega}$ containing the stabiliser of $\mathcal{P}$ also contains the almost stabiliser of $\mathcal{P}$.

\section{Containment}

In this section we consider the question of when a subsemigroup of $\Omega^{\Omega}$ is contained in a maximal subsemigroup. The analogous question has been considered for subgroups of the symmetric group; see, for example, [4,20,21. The proposition below is of particular interest here. In [4] it is shown that under certain set theoretic assumptions there exists a subgroup of $\operatorname{Sym}(\Omega)$ that is not contained in a maximal subgroup. However, such examples are difficult to find, and, roughly speaking, if a subgroup of $\operatorname{Sym}(\Omega)$ is large or small enough, then it is contained in a maximal subgroup.

It will be convenient to use the following notion: if $S$ is a semigroup and $T$ is a subset of $S$, then the relative rank of $T$ in $S$ is the least cardinality of a subset $U$ of $S$ such that $\langle T, U\rangle=S$.

Part (i) of the following proposition is a special case of Lemma 6.9 in Macpherson and Neumann [20], and parts (ii) and (iii) are Theorems 1.5 and 1.6 in Macpherson and Praeger [21. 
Proposition 3.1. Let $G$ be a subgroup of $\operatorname{Sym}(\Omega)$ satisfying any of the following:

(i) $G$ has finite relative rank in $\operatorname{Sym}(\Omega)$;

(ii) $|G| \leq|\Omega|$;

(iii) $|\Omega|$ is countable and there exists $t \in \mathbb{N}$ such that $G$ has infinitely many orbits on $\Omega^{t}$.

Then $G$ is contained in a maximal subgroup of $\operatorname{Sym}(\Omega)$.

Proposition 3.2 (Bergman-Shelah, Section 5 in [5]). Let $\Omega$ be countably infinite and let $G$ be a subgroup of $\operatorname{Sym}(\Omega)$ such that $G_{(\Sigma)}$ has an infinite orbit for all finite $\Sigma \subseteq \Omega$. Then $G$ has finite relative rank in $\operatorname{Sym}(\Omega)$ and hence is contained in a maximal subgroup.

We give an analogue of Proposition 3.1(i) and (ii) for subsemigroups of $\Omega^{\Omega}$.

Proposition 3.3. Let $S$ be a subsemigroup of $\Omega^{\Omega}$ satisfying either of the following:

(i) $S$ has finite relative rank in $\Omega^{\Omega}$;

(ii) $|S| \leq|\Omega|$.

Then $S$ is contained in a maximal subsemigroup of $\Omega^{\Omega}$.

Proof. (i) This is a straightforward consequence of Zorn's Lemma, analogous to the proof of Proposition 3.1(i).

(ii) Let $\iota$ be the cardinality of the set of injective elements of $S$ and let $\left\{f_{\alpha}: \alpha<\right.$ $\iota$ \} be those injective elements. Using transfinite induction for all ordinals $\alpha<\iota$ we may define

$$
x_{\alpha}, y_{\alpha} \in \Omega f_{\alpha} \backslash\left\{x_{\beta}, y_{\beta}: \beta<\alpha\right\}
$$

such that $x_{\alpha} \neq y_{\alpha}$. Let $T=\left\{f \in \Omega^{\Omega}: x_{\alpha} f=y_{\alpha} f(\forall \alpha<\iota)\right\}$. Then $\langle S, T\rangle$ is a proper subsemigroup of $\Omega^{\Omega}$, since every injective function in $\langle S, T\rangle$ belongs to $S$ and $|S| \leq|\Omega|$. Also if $\Sigma$ is a transversal of any $f \in T$ such that $|\Omega f|=|\Omega|$, then $\left\{\left.g\right|_{\Sigma}: g \in T\right\}=\Omega^{\Sigma}$. Hence if $h$ is any injective function in $\Omega^{\Omega}$ such that $\Omega h=\Sigma$, then $\langle S, T, h\rangle=\Omega^{\Omega}$. Hence $\langle S, T\rangle$, and so $S$, are contained in a maximal subsemigroup of $\Omega^{\Omega}$ by part (i).

A subgroup $G$ of $\operatorname{Sym}(\Omega)$ is highly transitive if for all $n \in \mathbb{N}$ and for all $\left(\alpha_{1}, \alpha_{2}, \ldots\right.$, $\left.\alpha_{n}\right),\left(\beta_{1}, \beta_{2}, \ldots, \beta_{n}\right) \in \Omega^{n}$, there exists $g \in G$ such that

$$
\left(\alpha_{1} g, \alpha_{2} g, \ldots, \alpha_{n} g\right)=\left(\beta_{1}, \beta_{2}, \ldots, \beta_{n}\right) .
$$

We give a new proof of the next theorem using Propositions 3.1 and 3.2

Theorem 3.4 (Macpherson \& Praeger [21]). Let $\Omega$ be countably infinite and let $G$ be a subgroup of $\operatorname{Sym}(\Omega)$ that is not highly transitive. Then $G$ is contained in a maximal subgroup of $\operatorname{Sym}(\Omega)$.

Proof. If $G$ is any subgroup of $\operatorname{Sym}(\Omega)$, then $G$ satisfies one of the following conditions:

(a) $G_{(\Sigma)}$ has an infinite orbit for all finite $\Sigma \subseteq \Omega$;

(b) there exists finite $\Sigma \subseteq \Omega$ such that every orbit of $G_{(\Sigma)}$ is finite.

Suppose that $G$ is a subgroup of $\operatorname{Sym}(\Omega)$ that is not highly transitive. If $G$ satisfies (a), then, by Proposition 3.2, $G$ is contained in a maximal subgroup.

If $G$ satisfies (b), then we may assume without loss of generality that $\Sigma=$ $\{0,1, \ldots, m-1\}$. Since every orbit of $G_{(\Sigma)}$ is finite, every orbit of $G$ on $\Omega^{m+1}$ contains only finitely many tuples of the form $(0,1, \ldots, m-1, n)$ where $n \in \mathbb{N}$. 
But there are infinitely many such tuples and so $G$ has infinitely many orbits on $\Omega^{m+1}$. Thus Proposition 3.1(iii) implies that $G$ is contained in a maximal subgroup of $\operatorname{Sym}(\Omega)$.

\section{Generating pairs}

In [14, Theorem 3.3] it is shown that $\operatorname{Sym}(\Omega)$ has relative rank 2 in $\Omega^{\Omega}$; that is, there exist $f, g \in \Omega^{\Omega}$ such that $\langle\operatorname{Sym}(\Omega), f, g\rangle=\Omega^{\Omega}$. Those pairs $f, g \in \Omega^{\Omega}$ satisfying this property are completely classified in the case that $|\Omega|$ is a regular cardinal; see [14, Theorem 4.1]. In this section, we recover this classification as a corollary to Theorem $\mathrm{A}$ and extend it to sets of arbitrary cardinality. Furthermore, we obtain analogous results where $\operatorname{Sym}(\Omega)$ is replaced by the stabiliser of a finite set, an ultrafilter, or a finite partition. We require the following straightforward lemma to obtain the corollaries in this section.

Lemma 4.1. Let $G$ be a subgroup of $\operatorname{Sym}(\Omega)$ containing $\operatorname{Sym}(\Omega)_{(\Sigma)}$ for some $\Sigma \subseteq \Omega$ such that $|\Omega \backslash \Sigma|=|\Omega|$ and let $H$ be any subset of $\Omega^{\Omega}$. Then $\langle G, H\rangle=\Omega^{\Omega}$ if and only if $H$ is not contained in any maximal subsemigroup of $\Omega^{\Omega}$ that contains $G$.

Proof. If $H$ is a subset of a maximal subsemigroup of $\Omega^{\Omega}$ containing $G$, then $\langle G, H\rangle$ is contained in that semigroup, and so $\langle G, H\rangle \neq \Omega^{\Omega}$. For the converse, 20, Lemma 2.4 states that if $U$ is any subgroup of $\operatorname{Sym}(\Omega)$ containing $\operatorname{Sym}(\Omega)_{(\Gamma)}$ for some moiety $\Gamma$ of $\Omega$, then there exists $x \in \operatorname{Sym}(\Omega)$ such that $\left\langle U, x, x^{-1}\right\rangle=\operatorname{Sym}(\Omega)$. It follows that $G$ has finite relative rank in $\operatorname{Sym}(\Omega)$. Hence, since $\operatorname{Sym}(\Omega)$ has finite relative rank in $\Omega^{\Omega}$ (by [14, Theorem 3.3] as stated above), any subsemigroup of $\Omega^{\Omega}$ containing $G$ has finite relative rank in $\Omega^{\Omega}$. It follows by Proposition 3.3 (i) that any proper subsemigroup of $\Omega^{\Omega}$ containing $G$ is contained in a maximal subsemigroup of $\Omega^{\Omega}$. Therefore if $H$ is not contained in any maximal subsemigroup containing $G$, then $\langle G, H\rangle=\Omega^{\Omega}$.

The following corollary of Theorem $\mathrm{A}$ and Lemma 4.1 extends [14, Theorem 4.1].

Corollary 4.2. Let $\Omega$ be any infinite set and let $f, g \in \Omega^{\Omega}$. Then $\langle\operatorname{Sym}(\Omega), f, g\rangle=$ $\Omega^{\Omega}$ if and only if (up to renaming $f$ and $g$ ) $f$ is injective, $d(f)=|\Omega|, g$ is surjective, and either:

(i) $|\Omega|$ is regular and $k(g,|\Omega|)=|\Omega|$; or

(ii) $|\Omega|$ is singular and $k(g, \nu)=|\Omega|$ for all $\nu<|\Omega|$.

Proof. By Lemma 4.1, it suffices to show that none of the maximal subsemigroups in Theorem $\mathrm{A}$ contains both $f$ and $g$ if and only if (up to renaming $f$ and $g$ ) $f$ is injective, $d(f)=|\Omega|, g$ is surjective, and either:

(i) $|\Omega|$ is regular and $k(g,|\Omega|)=|\Omega|$; or

(ii) $|\Omega|$ is singular and $k(g, \nu)=|\Omega|$ for all $\nu<|\Omega|$.

For the direct implication, if $\{f, g\}$ is not contained in $S_{1} \cup S_{2}$, then (up to renaming $f$ and $g) f$ is injective and $g$ is surjective. Hence $g \in S_{4}(\mu)$ and so $f \notin S_{4}(\mu)$ for all $\mu$. It follows that $d(f)=|\Omega|$. Regardless of the cardinality of $\Omega, f$ belongs to $S_{5}^{\prime} \subseteq S_{5}$. So, if $|\Omega|$ is regular, then $S_{5}$ is maximal, $g \notin S_{5}$, and $k(g,|\Omega|)=|\Omega|$. Similarly, if $|\Omega|$ is singular, then $k(g, \nu)=|\Omega|$ for all $\nu<|\Omega|$.

For the converse implication, it is easy to verify that $f \notin S_{2} \cup S_{4}(\mu)$ and $g \notin$ $S_{1} \cup S_{3}(\mu)$ for all infinite cardinals $\mu$ not greater than $|\Omega|$. If $|\Omega|$ is regular, then $g \notin S_{5}$, and if $|\Omega|$ is singular, then $g \notin S_{5}^{\prime}$. 
Analogous to Corollary 4.2, we can deduce from Theorem $\mathrm{B}$ a characterisation of those $f, g \in \Omega^{\Omega}$ that together with the pointwise stabiliser of a finite set generate $\Omega^{\Omega}$.

Corollary 4.3. Let $\Omega$ be any infinite set, let $\Sigma$ be a non-empty finite subset of $\Omega$, and let $f, g \in \Omega^{\Omega}$. Then the following are equivalent:

(I) $\left\langle\operatorname{Sym}(\Omega)_{(\Sigma)}, f, g\right\rangle=\Omega^{\Omega}$;

(II) $\left\langle\operatorname{Sym}(\Omega)_{\{\Gamma\}}, f, g\right\rangle=\Omega^{\Omega}$ for all $\Gamma \subseteq \Sigma$;

(III) $f$ and $g$ satisfy the conditions of Corollary 4.2 and for all non-empty $\Gamma \subseteq \Sigma$ one of the following holds:

(i) $\Gamma f \nsubseteq \Gamma$ and $\Gamma g^{-1} \nsubseteq \Gamma$;

(ii) $\Gamma g \nsubseteq \Gamma, \Gamma g^{-1} \nsubseteq \Gamma$, and $|\Gamma g|=|\Gamma|$;

(iii) $\Gamma f \nsubseteq \Gamma, \Gamma f^{-1} \nsubseteq \Gamma$, and $\Gamma \subseteq \Omega f$.

Proof. (I) $\Rightarrow(\mathrm{II})$ This implication follows immediately since $\operatorname{Sym}(\Omega)_{(\Sigma)} \subseteq \operatorname{Sym}(\Omega)_{\{\Gamma\}}$ for all $\Gamma \subseteq \Sigma$.

$(\mathrm{II}) \Rightarrow(\mathrm{III})$ Let $\Gamma$ be any non-empty subset of $\Sigma$. Since $\langle\operatorname{Sym}(\Omega), f, g\rangle \supseteq$ $\left\langle\operatorname{Sym}(\Omega)_{\{\Gamma\}}, f, g\right\rangle=\Omega^{\Omega}$, clearly $f$ and $g$ satisfy the conditions of Corollary 4.2, and $\{f, g\}$ is not contained in any proper subsemigroup of $\Omega^{\Omega}$ containing $\operatorname{Sym}(\Omega)_{\{\Gamma\}}$. In particular, $\{f, g\}$ is not a subset of $F_{1}\left(\Gamma,|\Omega|^{+}\right)$or $F_{2}\left(\Gamma,|\Omega|^{+}\right)$. If $f \notin F_{2}\left(\Gamma,|\Omega|^{+}\right)$ and $g \notin F_{1}\left(\Gamma,|\Omega|^{+}\right)$, then $\Gamma f \nsubseteq \Gamma$ and $\Gamma g^{-1} \nsubseteq \Gamma$ and so (i) holds. If $g \notin F_{2}\left(\Gamma,|\Omega|^{+}\right)$, then $|\Gamma g|=|\Gamma|$ and $\Gamma g \nsubseteq \Gamma$. But $g$ is surjective and so $\Gamma g^{-1} \nsubseteq \Gamma$, and so (ii) holds. If $f \notin F_{1}\left(\Gamma,|\Omega|^{+}\right)$, then $\Gamma \subseteq \Omega f$ and $\Gamma f^{-1} \nsubseteq \Gamma$. Hence, since $f$ is injective, $\Gamma f \nsubseteq \Gamma$ and (iii) holds.

(III) $\Rightarrow$ (I) Again by Lemma 4.1 it suffices to show that none of the maximal subsemigroups in Theorems $\mathrm{A}$ and $\mathrm{B}$ contain both $f$ and $g$.

Since $f$ and $g$ satisfy the conditions of Corollary 4.2, it follows that they are not contained in any of the semigroups from Theorem $\mathrm{A}$. Moreover, the same conditions imply that $f \notin F_{2}(\Gamma, \mu)$ and $g \notin F_{1}(\Gamma, \mu)$ for all $\mu \leq|\Omega|$. If (i) holds, then $f \notin F_{2}\left(\Gamma,|\Omega|^{+}\right)$and $g \notin F_{1}\left(\Gamma,|\Omega|^{+}\right)$. If (ii) holds, then $g \notin F_{1}\left(\Gamma,|\Omega|^{+}\right) \cup F_{2}\left(\Gamma,|\Omega|^{+}\right)$, and if (iii) holds, then $f \notin F_{1}\left(\Gamma,|\Omega|^{+}\right) \cup F_{2}\left(\Gamma,|\Omega|^{+}\right)$.

In the next corollary we characterise the pairs of functions that together with the stabiliser of an ultrafilter generate $\Omega^{\Omega}$. The statement of this result is similar to that of Corollary 4.3 .

Corollary 4.4. Let $\Omega$ be any infinite set, let $\mathcal{F}$ be an ultrafilter on $\Omega$, and let $f, g \in \Omega^{\Omega}$. Then $\left\langle\operatorname{Sym}(\Omega)_{\{\mathcal{F}\}}, f, g\right\rangle=\Omega^{\Omega}$ if and only if $f$ and $g$ satisfy the conditions of Corollary 4.2 and there exist $\Sigma \in \mathcal{F}$ and $\Gamma \notin \mathcal{F}$ such that one of the following holds:

(i) $\Sigma f \notin \mathcal{F}$ and $\Gamma g \in \mathcal{F}$;

(ii) $\Sigma g \notin \mathcal{F}, c\left(\left.g\right|_{\Sigma}\right)=0$, and $\Gamma g \in \mathcal{F}$;

(iii) $\Sigma f \notin \mathcal{F}$ and $\Gamma f \in \mathcal{F}$.

Proof. If $\mathcal{F}$ is a principal ultrafilter, say generated by $\{\alpha\}$, then $\operatorname{Sym}(\Omega)_{\{\mathcal{F}\}}=$ $\operatorname{Sym}(\Omega)_{(\{\alpha\})}$ and the result follows by Corollary 4.3 .

Suppose that $\mathcal{F}$ is a non-principal ultrafilter. Recall that $\operatorname{Sym}(\Omega)_{\{\mathcal{F}\}}$ contains the pointwise stabiliser of any $\Sigma \in \mathcal{F}$. Let $\kappa$ denote the least cardinality of a set in $\mathcal{F}$. If $\kappa<|\Omega|$, then there exists $\Sigma \in \mathcal{F}$ such that $|\Sigma|=\kappa$ and so $|\Omega \backslash \Sigma|=|\Omega|$. Suppose that $\kappa=|\Omega|$. Then if $\Sigma \in \mathcal{F}$ is such that $|\Omega \backslash \Sigma|<|\Omega|$ and $\Gamma$ is a moiety of $\Sigma$ (and hence in $\Omega$ ), then either $\Gamma \in \mathcal{F}$ or $\Sigma \backslash \Gamma \in \mathcal{F}$ (since otherwise $\Omega \backslash \Sigma \in \mathcal{F}$ and 
$|\Omega \backslash \Sigma|<\kappa$, which is a contradiction). In either case, it follows that $\operatorname{Sym}(\Omega)_{\{\mathcal{F}\}}$ contains the pointwise stabiliser of some $\Sigma \in \mathcal{F}$ such that $|\Omega \backslash \Sigma|=|\Omega|$.

Therefore by Lemma 4.1 and Theorem $\mathrm{C}$ it follows that $\left\langle\operatorname{Sym}(\Omega)_{\{\mathcal{F}\}}, f, g\right\rangle=\Omega^{\Omega}$ if and only if $\{f, g\}$ is not a subset of $U_{1}(\mathcal{F}, \mu) \cup U_{2}(\mathcal{F}, \mu)$ for any cardinal $\mu$ such that $\kappa<\mu \leq|\Omega|^{+}$.

$(\Rightarrow)$ Since $\langle\operatorname{Sym}(\Omega), f, g\rangle \supseteq\left\langle\operatorname{Sym}(\Omega)_{\{\mathcal{F}\}}, f, g\right\rangle=\Omega^{\Omega}$, clearly $f$ and $g$ satisfy the conditions of Corollary 4.2. From the discussion above, it follows that, in particular, $\{f, g\} \nsubseteq U_{1}\left(\mathcal{F},|\Omega|^{+}\right) \cup U_{2}\left(\mathcal{F},|\Omega|^{+}\right)$. If $g \notin U_{1}\left(\mathcal{F},|\Omega|^{+}\right)$and $f \notin U_{2}\left(\mathcal{F},|\Omega|^{+}\right)$, then there exists $\Sigma \in \mathcal{F}$ and $\Gamma \notin \mathcal{F}$ such that $\Sigma f \notin \mathcal{F}$ and $\Gamma g \in \mathcal{F}$, in which case (i) holds. If $f \notin U_{1}\left(\mathcal{F},|\Omega|^{+}\right)$, then there exists $\Gamma \notin \mathcal{F}$ such that $\Gamma f \in \mathcal{F}$. It follows that $\Omega \backslash \Gamma \in \mathcal{F}$ and $(\Omega \backslash \Gamma) f \subseteq(\Omega \backslash \Gamma f) \notin \mathcal{F}$, and so $(\Omega \backslash \Gamma) f \notin \mathcal{F}$, which implies (iii) holds. If $g \notin U_{2}\left(\mathcal{F},|\Omega|^{+}\right)$, then there exists $\Sigma \in \mathcal{F}$ such that $\Sigma g \notin \mathcal{F}$ and $c\left(\left.g\right|_{\Sigma}\right)=0$. But $g$ is surjective and so $(\Omega \backslash \Sigma) g \supseteq \Omega \backslash(\Sigma g) \in \mathcal{F}$. Thus $\Omega \backslash \Sigma \notin \mathcal{F}$ but $(\Omega \backslash \Sigma) g \in \mathcal{F}$ and so (ii) holds.

$(\Leftarrow)$ If $\mu \leq|\Omega|$, then, since $f$ and $g$ satisfy the conditions of Corollary 4.2, it follows that $f \notin U_{2}(\mathcal{F}, \mu)$ and $g \notin U_{1}(\mathcal{F}, \mu)$. Hence it suffices to show that $\{f, g\} \nsubseteq U_{1}\left(\mathcal{F},|\Omega|^{+}\right) \cup U_{2}\left(\mathcal{F},|\Omega|^{+}\right)$if one of (i), (ii), or (iii) holds. It is easy to verify that if (i) holds, then $f \notin U_{2}\left(\mathcal{F},|\Omega|^{+}\right)$and $g \notin U_{1}\left(\mathcal{F},|\Omega|^{+}\right)$; and if (ii) or (iii) holds, then $g \notin U_{1}\left(\mathcal{F},|\Omega|^{+}\right) \cup U_{2}\left(\mathcal{F},|\Omega|^{+}\right)$or $f \notin U_{1}\left(\mathcal{F},|\Omega|^{+}\right) \cup U_{2}\left(\mathcal{F},|\Omega|^{+}\right)$, respectively.

As above, Theorem $\mathrm{D}$ can be used to characterise those $f, g \in \Omega^{\Omega}$ that together with either $\operatorname{Stab}(\mathcal{P})$ or $\operatorname{AStab}(\mathcal{P})$ generate $\Omega^{\Omega}$.

Corollary 4.5. Let $\Omega$ be any infinite set, let $\mathcal{P}=\left\{\Sigma_{0}, \Sigma_{1}, \ldots, \Sigma_{n-1}\right\}, n \geq 2$, be a finite partition of $\Omega$, and let $f, g \in \Omega^{\Omega}$. Then the following are equivalent:

(I) $\langle\operatorname{Stab}(\mathcal{P}), f, g\rangle=\Omega^{\Omega}$;

(II) $\langle\operatorname{AStab}(\mathcal{P}), f, g\rangle=\Omega^{\Omega}$;

(III) $f$ and $g$ satisfy the conditions of Corollary 4.2 and one of the following holds:

(i) $\rho_{f}, \rho_{g} \notin \operatorname{Sym}(n)$;

(ii) $\rho_{f} \notin \operatorname{Sym}(n)$ and $\rho_{f}^{-1}$ is total;

(iii) $\rho_{g} \notin \operatorname{Sym}(n)$ and $\rho_{g}$ is total.

Proof. (I) $\Rightarrow$ (II) This implication follows immediately $\operatorname{since} \operatorname{Stab}(\mathcal{P})$ is a $\operatorname{subgroup}$ of $\operatorname{AStab}(\mathcal{P})$.

(II) $\Rightarrow$ (III) If $\Sigma=\Sigma_{1} \cup \cdots \cup \Sigma_{n-1}$, then $\operatorname{Stab}(\mathcal{P})$, and hence $\operatorname{AStab}(\mathcal{P})$, contains the pointwise stabiliser of $\Sigma$ in $\operatorname{Sym}(\Omega)$. Hence by Lemma 4.1, $\langle\operatorname{AStab}(\mathcal{P}), f, g\rangle=$ $\Omega^{\Omega}$ implies that $\{f, g\}$ is not a subset of $A_{1}(\mathcal{P})$ or $A_{2}(\mathcal{P})$. If $f \notin A_{1}(\mathcal{P})$ and $g \notin A_{2}(\mathcal{P})$, then $\rho_{f}, \rho_{g} \notin \operatorname{Sym}(n)$ and (i) holds. If $f \notin A_{2}(\mathcal{P})$, then $\rho_{f} \notin \operatorname{Sym}(n)$ and $\rho_{f}^{-1}$ is total and (ii) holds. If $g \notin A_{1}(\mathcal{P})$, then $\rho_{g} \notin \operatorname{Sym}(n)$ and $\rho_{g}$ is total and we are in case (iii).

$(\mathrm{III}) \Rightarrow(\mathrm{I})$ Again by Lemma 4.1, to prove that $\langle\operatorname{Stab}(\mathcal{P}), f, g\rangle=\Omega^{\Omega}$, it suffices to show that none of the maximal subsemigroups in Theorems $\mathrm{A}$ and $\mathrm{D}$ contain both $f$ and $g$.

Since $f$ and $g$ satisfy the conditions of Corollary 4.2, it follows that they are not contained in any of the semigroups from Theorem A. If (i) holds, then $f \notin$ $A_{1}(\mathcal{P})$ and $g \notin A_{2}(\mathcal{P})$; if (ii) holds, then, since $f$ is injective, $\rho_{f}$ is total and so $f \notin A_{1}(\mathcal{P}) \cup A_{2}(\mathcal{P})$; and if (iii) holds, then, since $g$ is surjective, $\rho_{g}^{-1}$ is total and so $g \notin A_{1}(\mathcal{P}) \cup A_{2}(\mathcal{P})$. 


\section{INVERSES AND PARAMETERS OF MAPPINGS}

In this section we present several technical results which we will use repeatedly throughout the paper.

We begin by considering the semigroup theoretic inverses of mappings in $\Omega^{\Omega}$. Roughly speaking, the proofs of the main theorems are in two parts and Corollary 5.3 will imply that one part is a corollary of the other. More precisely, the majority of the proof of, say, Theorem $\mathrm{B}$ consists of showing the following. If $U$ is a subsemigroup of $\Omega^{\Omega}$ that is not contained in any of the semigroups listed in Theorems $\mathrm{A}$ or $\mathrm{B}$ but that does contain the stabiliser of a non-empty finite subset of $\Omega$, then $U=\Omega^{\Omega}$. The stabiliser contains the symmetric group on an infinite subset $\Sigma$ of $\Omega$. The two parts of the proof, referred to above, are to construct an injective mapping in $U$ with image contained in $\Sigma$ and a surjective mapping in $U$ mapping $\Sigma$ onto $\Omega$. Using Corollary 5.3 , the existence of the surjective mapping is a consequence of the existence of the injective mapping. The proofs of Theorems $\mathrm{A}$. C] and D follow a similar strategy.

If $S$ is a semigroup and $s \in S$, then $t \in S$ is an inverse of $s$ if $s t s=s$ and $t s t=t$. Clearly, $t$ is an inverse for $s$ if and only if $s$ is an inverse for $t$. If $f, f^{\prime} \in \Omega^{\Omega}$, then $f^{\prime}$ is an inverse for $f$ if and only if $\Omega f^{\prime}$ is a transversal of $f$ and $f f^{\prime}$ is the identity on $\Omega f^{\prime}$. Note that if $f, f^{\prime} \in \Omega^{\Omega}$ are inverses, then $c(f)=d\left(f^{\prime}\right)$.

In general, the composition $g^{\prime} f^{\prime}$ of inverses of $g$ and $f$ is not an inverse of the composite $f g$. However, for certain composites $g^{\prime} f^{\prime}$ is an inverse of $f g$.

Lemma 5.1. Let $u_{0}, u_{1}, \ldots, u_{n} \in \Omega^{\Omega}$ be arbitrary and let $u_{i}^{\prime}$ be an inverse of $u_{i}$ for all $i \in\{0,1, \ldots, n\}$. If $\Omega u_{0}^{\prime} u_{1}^{\prime} \cdots u_{i-1}^{\prime} \subseteq \Omega u_{i}$ for all $i \in\{1, \ldots, n\}$, then $u_{0}^{\prime} u_{1}^{\prime} \cdots u_{n}^{\prime}$ and $u_{n} \cdots u_{1} u_{0}$ are inverses.

Proof. We show that $u_{n} \cdots u_{1} u_{0}$ is an inverse of $u_{0}^{\prime} u_{1}^{\prime} \cdots u_{n}^{\prime}$ by showing that $\Omega u_{n} \cdots u_{1} u_{0}$ is a transversal of $u_{0}^{\prime} u_{1}^{\prime} \cdots u_{n}^{\prime}$ and $u_{0}^{\prime} u_{1}^{\prime} \cdots u_{n}^{\prime} u_{n} \cdots u_{1} u_{0}$ is the identity on $\Omega u_{n} \cdots u_{1} u_{0}$.

Since $\Omega u_{0}^{\prime} u_{1}^{\prime} \cdots u_{i-1}^{\prime}$ is contained in the transversal $\Omega u_{i}$ of $u_{i}^{\prime}$ for all $i \in\{1, \ldots, n\}$, it follows that $u_{1}^{\prime} \cdots u_{n}^{\prime}$ is injective on $\Omega u_{0}^{\prime}$. Hence the transversal $\Omega u_{0}$ of $u_{0}^{\prime}$ is also a transversal of $u_{0}^{\prime} u_{1}^{\prime} \cdots u_{n}^{\prime}$.

If $x \in \Omega$, then $x u_{0}^{\prime} u_{1}^{\prime} \cdots u_{i-1}^{\prime} \in \Omega u_{i}$ for all $i \in\{1, \ldots, n\}$. Since $u_{i}^{\prime} u_{i}$ is the identity on $\Omega u_{i}$, it follows that $x u_{0}^{\prime} u_{1}^{\prime} \cdots u_{i-1}^{\prime} u_{i}^{\prime} u_{i}=x u_{0}^{\prime} u_{1}^{\prime} \cdots u_{i-1}^{\prime}$ for all $x \in \Omega$.

Applying this $n$ times we obtain

$$
x u_{0}^{\prime} u_{1}^{\prime} \cdots u_{n}^{\prime} u_{n} \cdots u_{1} u_{0}=x u_{0}^{\prime} u_{0}
$$

for all $x \in \Omega$. In particular, if $x \in \Omega u_{0}$, then $x u_{0}^{\prime} u_{1}^{\prime} \cdots u_{n}^{\prime} u_{n} \cdots u_{1} u_{0}=x$.

Certainly, $\Omega u_{n} \cdots u_{0} \subseteq \Omega u_{0}$ and since $u_{0}^{\prime} u_{1}^{\prime} \cdots u_{n}^{\prime} u_{n} \cdots u_{1} u_{0}$ is the identity on $\Omega u_{0}$, it follows that $\Omega u_{n} \cdots u_{0} \supseteq \Omega u_{0}^{\prime} u_{1}^{\prime} \cdots u_{n}^{\prime} u_{n} \cdots u_{1} u_{0} \supseteq \Omega u_{0}$.

Definition 5.2. Let $V \subseteq \Omega^{\Omega}$ and let $\Lambda: V \rightarrow \mathcal{P}(\Omega)$ be such that $\Lambda(v)$ is a transversal of $v$ for all $v \in V$. We refer to such a $\Lambda$ as an assignment of transversals for $V$. Then the set of products $v_{0} v_{1} \cdots v_{n} \in\langle V\rangle$ such that $v_{i} \in V$ and $\Omega v_{0} \cdots v_{i-1} \subseteq \Lambda\left(v_{i}\right)$ for all $i \in\{1, \ldots, n\}$ is denoted by $\mathcal{C}(V, \Lambda)$.

Corollary 5.3. Let $U \subseteq \Omega^{\Omega}$, let $u^{\prime} \in \Omega^{\Omega}$ be an inverse of $u$ for every $u \in U$, let $U^{\prime}=\left\{u^{\prime}: u \in U\right\}$, and let $\Lambda: U^{\prime} \rightarrow \mathcal{P}(\Omega)$ be defined by $\Lambda\left(u^{\prime}\right)=\Omega u$. Then every element of $\mathcal{C}\left(U^{\prime}, \Lambda\right)$ has an inverse in $\langle U\rangle$. 
Proof. Let $u_{0}^{\prime} u_{1}^{\prime} \cdots, u_{n}^{\prime} \in \mathcal{C}\left(U^{\prime}, \Lambda\right)$. Then $\Omega u_{0}^{\prime} u_{1}^{\prime} \cdots u_{i-1}^{\prime} \subseteq \Lambda\left(u_{i}^{\prime}\right)=\Omega u_{i}$ for all $i \in\{1, \ldots, n\}$. Thus, by Lemma 5.1, $u_{n} \cdots u_{0} \in\langle U\rangle$ is an inverse of $u_{0}^{\prime} \cdots u_{n}^{\prime}$.

We will make repeated use of the following lemma, which is similar to Lemma 2.1 in 14 .

Lemma 5.4. Let $\Omega$ be any infinite set, let $\mu$ be an infinite cardinal such that $\mu \leq|\Omega|$, and let $f, g \in \Omega^{\Omega}$. Then the following hold:

(i) if $\mu$ is a regular cardinal, then $k(f g, \mu) \leq k(f, \mu)+k(g, \mu)$;

(ii) $d(g) \leq d(f g) \leq d(f)+d(g)$;

(iii) if $g$ is injective (i.e. $c(g)=0)$, then $d(f g)=d(f)+d(g)$;

(iv) $c(f) \leq c(f g) \leq c(f)+c(g)$;

(v) if $f$ is surjective (i.e. $d(f)=0)$, then $c(f g)=c(f)+c(g)$;

(vi) if $c(g)<\mu \leq d(f)$, then $d(f g) \geq \mu$;

(vii) if $d(f)<\mu \leq c(g)$, then $c(f g) \geq \mu$.

Proof. (i) Let $\alpha \in \Omega$. Then

$$
\alpha(f g)^{-1}=\bigcup_{\beta \in \alpha g^{-1}} \beta f^{-1} .
$$

If $\left|\alpha g^{-1}\right|<\mu$ and $\left|\beta f^{-1}\right|<\mu$ for all $\beta \in \alpha g^{-1}$, then, since $\mu$ is regular, $\left|\alpha(f g)^{-1}\right|<\mu$. Hence

$$
\begin{aligned}
k(f g, \mu) & =\left|\left\{\alpha \in \Omega:\left|\alpha(f g)^{-1}\right| \geq \mu\right\}\right| \\
& \leq\left|\left\{\alpha \in \Omega:\left(\exists \beta \in \alpha g^{-1}\right)\left(\left|\beta f^{-1}\right| \geq \mu\right)\right\}\right|+\left|\left\{\alpha \in \Omega:\left|\alpha g^{-1}\right| \geq \mu\right\}\right| \\
& \leq k(f, \mu)+k(g, \mu),
\end{aligned}
$$

as required.

(ii) It is straightforward to see that

$$
\Omega \backslash \Omega g \subseteq \Omega \backslash \Omega f g \subseteq(\Omega \backslash \Omega f) g \cup(\Omega \backslash \Omega g),
$$

and so $d(g) \leq d(f g) \leq|(\Omega \backslash \Omega f) g|+d(g) \leq d(f)+d(g)$.

(iii) If $c(g)=0$, then

$$
\Omega \backslash \Omega f g=(\Omega \backslash \Omega g) \cup(\Omega g \backslash \Omega f g)=(\Omega \backslash \Omega f) g \cup(\Omega \backslash \Omega g)
$$

and $|(\Omega \backslash \Omega f) g|=|\Omega \backslash \Omega f|=d(f)$. Hence $d(f g)=d(f)+d(g)$, as required.

(iv) Let $\Sigma \subseteq \Omega$ be a transversal of $f$. Then there exists $\Sigma^{\prime} \subseteq \Sigma$ such that $\Sigma^{\prime}$ is a transversal of $f g$. Hence $c(f) \leq c(f g)$. Also $c(f g)=\left|\Omega \backslash \Sigma^{\prime}\right|=|\Omega \backslash \Sigma|+\left|\Sigma \backslash \Sigma^{\prime}\right|=$ $c(f)+\left|\Sigma \backslash \Sigma^{\prime}\right|$, and so it suffices to show that $\left|\Sigma \backslash \Sigma^{\prime}\right| \leq c(g)$. Let $\Gamma$ be any transversal of $g$ such that $\Sigma^{\prime} f \subseteq \Gamma$. If $\alpha \in \Sigma \backslash \Sigma^{\prime}$, then there exists $\beta \in \Sigma^{\prime}$ such that $(\alpha) f g=(\beta) f g$. Since $f$ is injective on $\Sigma, \alpha f \neq \beta f$. But $\beta f \in \Sigma^{\prime} f \subseteq \Gamma$ and so $\alpha f \notin \Gamma$. Thus $\left(\Sigma \backslash \Sigma^{\prime}\right) f \subseteq \Omega \backslash \Gamma$ and so $\left|\Sigma \backslash \Sigma^{\prime}\right|=\left|\left(\Sigma \backslash \Sigma^{\prime}\right) f\right| \leq|\Omega \backslash \Gamma|=c(g)$, as required.

(v) Let $\Sigma, \Sigma^{\prime}$, and $\Gamma$ be as in part (iv). If $d(f)=0$, then $\Sigma f=\Omega$. But we saw in part (iv) that $\left(\Sigma \backslash \Sigma^{\prime}\right) f \subseteq \Omega \backslash \Gamma$ and $\Sigma^{\prime} f \subseteq \Gamma$, and so in this case $\left(\Sigma \backslash \Sigma^{\prime}\right) f=\Omega \backslash \Gamma$ and $\Sigma^{\prime} f=\Gamma$. Therefore $c(f g)=\left|\Omega \backslash \Sigma^{\prime}\right|=|\Omega \backslash \Sigma|+\left|\Sigma \backslash \Sigma^{\prime}\right|=|\Omega \backslash \Sigma|+|\Omega \backslash \Gamma|=c(f)+c(g)$.

(vi) If $\Sigma$ is any transversal of $g$, then, by assumption, $|\Omega \backslash \Sigma|=c(g)<\mu$ and $|\Omega \backslash \Omega f|=d(f) \geq \mu$. Hence $|\Sigma \cap(\Omega \backslash \Omega f)| \geq \mu$. If $\alpha \in \Sigma \cap(\Omega \backslash \Omega f)$ is such that $\alpha g \in \Omega f g$, then there exists $\beta \in \Omega f$ such that $\alpha g=\beta g$. So, since $c(g)<\mu$,

$$
|\{\alpha \in \Sigma \cap(\Omega \backslash \Omega f): \alpha g \in \Omega f g\}|<\mu .
$$


Therefore $|\{\alpha \in \Sigma \cap(\Omega \backslash \Omega f): \alpha g \notin \Omega f g\}| \geq \mu$, and so $|\Omega \backslash \Omega f g| \geq|\{\alpha \in \Sigma \cap(\Omega \backslash \Omega f): \alpha g \notin \Omega f g\} g|=|\{\alpha \in \Sigma \cap(\Omega \backslash \Omega f): \alpha g \notin \Omega f g\}| \geq \mu$, as required.

(vii) As in the proof of (iv), let $\Sigma$ be a transversal of $f$, let $\Sigma^{\prime} \subseteq \Sigma$ be a transversal of $f g$, and let $\Gamma$ be a transversal of $g$ such that $\Sigma^{\prime} f \subseteq \Gamma$. By assumption, $|\Omega \backslash \Sigma f|=|\Omega \backslash \Omega f|=d(f)<\mu$ and $|\Omega \backslash \Gamma|=c(g) \geq \mu$. Hence $|\Sigma f \cap(\Omega \backslash \Gamma)| \geq \mu$. Since $\Sigma^{\prime} f \subseteq \Gamma$ and, again as in the proof of (iv), $\left(\Sigma \backslash \Sigma^{\prime}\right) f \subseteq \Omega \backslash \Gamma$, it follows that $\Sigma f \cap(\Omega \backslash \Gamma)=\left(\Sigma \backslash \Sigma^{\prime}\right) f$. Thus

$$
\mu \leq|\Sigma f \cap(\Omega \backslash \Gamma)|=\left|\left(\Sigma \backslash \Sigma^{\prime}\right) f\right|=\left|\Sigma \backslash \Sigma^{\prime}\right| \leq\left|\Omega \backslash \Sigma^{\prime}\right|=c(f g),
$$

as required.

\section{The symmetric group - The Proof of Theorem A}

In this section, we give the proof of Theorem $\mathrm{A}$. We require the following result from [14, Theorem 3.3].

Theorem 6.1. Let $\Omega$ be an infinite set and let $f, g \in \Omega^{\Omega}$ be such that $f$ is injective, $g$ is surjective, and $d(f)=k(g,|\Omega|)=|\Omega|$. Then $\langle\operatorname{Sym}(\Omega), f, g\rangle=\Omega^{\Omega}$.

Recall that a subset $\Sigma$ of an infinite set $\Gamma$ is a moiety of $\Gamma$ if $|\Sigma|=|\Gamma \backslash \Sigma|=|\Gamma|$.

Lemma 6.2. Let $\Omega$ be any set of singular cardinality and let $g \in \Omega^{\Omega}$ be such that $k(g, \mu)=|\Omega|$ for all $\mu<|\Omega|$. Then there exists $a \in \operatorname{Sym}(\Omega)$ such that $k(g a g,|\Omega|)=$ $|\Omega|$.

Proof. Since $|\Omega|$ is singular, there exist $\kappa<|\Omega|$ and $\Omega_{\mu} \subseteq \Omega$ such that $\left|\Omega_{\mu}\right|<|\Omega|$ for all $\mu<\kappa$ and $\Omega=\bigcup_{\mu<\kappa} \Omega_{\mu}$. Let $\Sigma$ be a moiety of $\left\{\alpha \in \Omega:\left|\alpha g^{-1}\right| \geq \kappa\right\}$, let $\{\beta(\alpha, \mu) \in \Omega: \mu<\kappa\} \subseteq \alpha g^{-1}$ for all $\alpha \in \Sigma$, where $\beta(\alpha, \mu) \neq \beta(\alpha, \nu)$ if $\mu \neq \nu$, and let $\Sigma^{\prime}=\bigcup_{\alpha \in \Sigma}\{\beta(\alpha, \mu) \in \Omega: \mu<\kappa\}$.

We next show that there exists a moiety $\Gamma$ of $\Omega$ such that $\mid\left\{\alpha \in \Gamma:\left|\alpha g^{-1}\right| \geq\right.$ $\mu\}|=| \Omega \mid$ for all $\mu<|\Omega|$. In fact, if $\Omega$ is arbitrarily partitioned into moieties $\Gamma_{1}$ and $\Gamma_{2}$, then one or the other of these sets has the required property. To see this, suppose that there exists $\nu<|\Omega|$ such that $\left|\left\{\alpha \in \Gamma_{1}:\left|\alpha g^{-1}\right| \geq \nu\right\}\right|<|\Omega|$. If $\mu$ is a cardinal such that $\nu \leq \mu<|\Omega|$, then $k(g, \mu)=\left|\left\{\alpha \in \Omega:\left|\alpha g^{-1}\right| \geq \mu\right\}\right|=|\Omega|$. But

$$
\left\{\alpha \in \Omega:\left|\alpha g^{-1}\right| \geq \mu\right\}=\left\{\alpha \in \Gamma_{1}:\left|\alpha g^{-1}\right| \geq \mu\right\} \cup\left\{\alpha \in \Gamma_{2}:\left|\alpha g^{-1}\right| \geq \mu\right\}
$$

and so $\left|\left\{\alpha \in \Gamma_{2}:\left|\alpha g^{-1}\right| \geq \mu\right\}\right|=|\Omega|$. So we now fix $\Gamma$ with the above property.

Assume that $\Sigma \times \kappa$ is well-ordered. We define, by transfinite recursion, distinct $\gamma(\alpha, \mu) \in \Gamma$ such that $\left|\gamma(\alpha, \mu) g^{-1}\right| \geq\left|\Omega_{\mu}\right|$ for all $(\alpha, \mu) \in \Sigma \times \kappa$ as follows. Let $(\alpha, \mu) \in \Sigma \times \kappa$ and let

$$
\Gamma^{\prime}=\{\gamma(\beta, \nu):(\beta, \nu)<(\alpha, \mu)\} .
$$

Then $\left|\left\{\gamma \in \Gamma:\left|\gamma g^{-1}\right| \geq\left|\Omega_{\mu}\right|\right\}\right|=|\Omega|$ and $\left|\Gamma^{\prime}\right|<|\Sigma \times \kappa|=|\Omega|$. So, we may choose $\gamma(\alpha, \mu)$ to be any element in the set $\left\{\gamma \in \Gamma \backslash \Gamma^{\prime}:\left|\gamma g^{-1}\right| \geq\left|\Omega_{\mu}\right|\right\}$ which is of cardinality $|\Omega|$.

Since $\Gamma$ and $\Sigma^{\prime}$ are moieties, there exists $a \in \operatorname{Sym}(\Omega)$ such that

$$
(\gamma(\alpha, \mu)) a=\beta(\alpha, \mu)
$$

for all $(\alpha, \mu) \in \Sigma \times \kappa$. Therefore, for any $\alpha \in \Sigma$,

$$
\alpha(g a g)^{-1} \supseteq \bigcup_{\mu<\kappa} \beta(\alpha, \mu) a^{-1} g^{-1}=\bigcup_{\mu<\kappa} \gamma(\alpha, \mu) g^{-1}
$$




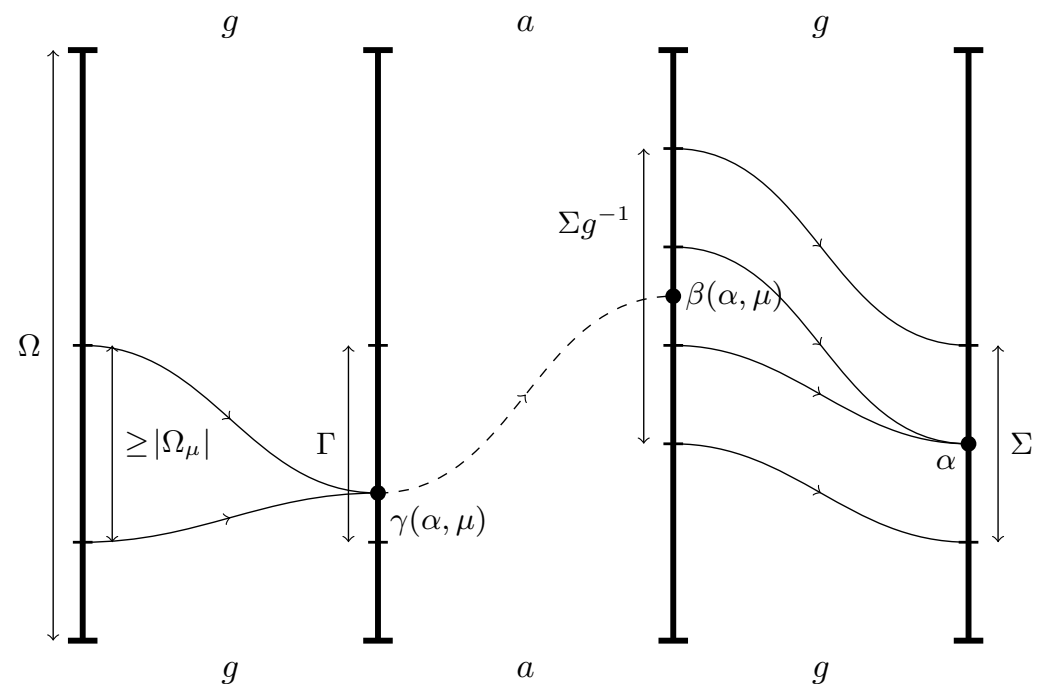

Figure 1. The composite gag in the proof of Lemma 6.2

and so $\mid \alpha(\text { gag })^{-1}|\geq| \bigcup_{\mu<\kappa} \Omega_{\mu}|=| \Omega \mid$; see Figure1. Since $|\Sigma|=|\Omega|$, it follows that $k($ gag,$|\Omega|)=|\Omega|$, as required.

Theorem A (Gavrilov [11, Pinsker [24]). Let $\Omega$ be any infinite set. If $|\Omega|$ is a regular cardinal, then the maximal subsemigroups of $\Omega^{\Omega}$ containing $\operatorname{Sym}(\Omega)$ are:

$$
\begin{aligned}
S_{1} & =\left\{f \in \Omega^{\Omega}: c(f)=0 \text { or } d(f)>0\right\}, \\
S_{2} & =\left\{f \in \Omega^{\Omega}: c(f)>0 \text { or } d(f)=0\right\}, \\
S_{3}(\mu) & =\left\{f \in \Omega^{\Omega}: c(f)<\mu \text { or } d(f) \geq \mu\right\}, \\
S_{4}(\mu) & =\left\{f \in \Omega^{\Omega}: c(f) \geq \mu \text { or } d(f)<\mu\right\}, \\
S_{5} & =\left\{f \in \Omega^{\Omega}: k(f,|\Omega|)<|\Omega|\right\},
\end{aligned}
$$

where $\mu$ is any infinite cardinal not greater than $|\Omega|$.

If $|\Omega|$ is a singular cardinal, then the maximal subsemigroups of $\Omega^{\Omega}$ containing $\operatorname{Sym}(\Omega)$ are $S_{1}, S_{2}, S_{3}(\mu), S_{4}(\mu)$ where $\mu$ is any infinite cardinal not greater than $|\Omega|$, and

$$
S_{5}^{\prime}=\left\{f \in \Omega^{\Omega}:(\exists \nu<|\Omega|)(k(f, \nu)<|\Omega|)\right\} .
$$

If $\Omega$ is any infinite set, then Lemma 5.4 can be used to show that $S_{1}, S_{2}, S_{3}(\mu)$, and $S_{4}(\mu)$ are semigroups for all infinite $\mu \leq|\Omega|$. In particular, parts (ii), (iii), and (iv) show this for $S_{1}$; (ii), (iv), (v) show this for $S_{2}$; (ii), (iv), (vi) show this for $S_{3}(\mu)$; and (ii), (iv), (vii) show this for $S_{4}(\mu)$. It is also straightforward to verify that none of $S_{1}, S_{2}, S_{3}(\mu), S_{4}(\nu)$, with $\mu, \nu \leq|\Omega|$ infinite cardinals, are contained in any of the others.

If $|\Omega|$ is regular, then Lemma 5.4(i) shows that $S_{5}$ is a semigroup. If $|\Omega|$ is singular, then $S_{5}$ is a generating set for $\Omega^{\Omega}$ and, in particular, not a semigroup. Regardless of the nature of $|\Omega|, S_{5}^{\prime}$ is contained in $S_{5}$. However, $S_{5}$ and $S_{5}^{\prime}$ are not contained in, and do not contain, any of $S_{1}, S_{2}, S_{3}(\mu)$, and $S_{4}(\mu)$ for any $\mu$. 
To show that $S_{5}^{\prime}$ is a semigroup in the case that $|\Omega|$ is a singular cardinal, let $f, g \in S_{5}^{\prime}$. Then there exists $\mu, \nu<|\Omega|$ such that $k(f, \mu)<|\Omega|$ and $k(g, \nu)<|\Omega|$. Let $\kappa=\max \{\mu, \nu\}$. If $\kappa^{+}$denotes the successor of $\kappa$, then $\kappa^{+}<|\Omega|$ since $|\Omega|$ is singular. Since $k\left(f, \kappa^{+}\right), k\left(g, \kappa^{+}\right)<|\Omega|$ and $\kappa^{+}$is regular, it follows, by Lemma 5.4(i), that $k\left(f g, \kappa^{+}\right) \leq k\left(f, \kappa^{+}\right)+k\left(g, \kappa^{+}\right)<|\Omega|$. Hence $f g \in S_{5}^{\prime}$ and $S_{5}^{\prime}$ is a semigroup.

We require Lemmas 6.3 and 6.4 below to complete the proof of Theorem $\mathrm{A}$, They are stated in far greater generality than required in this section because we will use them again in later sections.

If $a \in \operatorname{Sym}(\Omega)$, then we denote the set $\{\alpha \in \Omega: \alpha a \neq \alpha\}$ by $\operatorname{supp}(a)$ and $\operatorname{refer}$ to this set as the support of $a$.

Lemma 6.3. Let $U$ be a subset of $\Omega^{\Omega}$, which is not contained in $S_{2}$ or $S_{4}(\mu)$ for any infinite $\mu \leq|\Omega|$, let $\Lambda$ be any assignment of transversals for $U$ (as defined in Definition [5.2), and let $\kappa$ be any cardinal such that $\aleph_{0} \leq \kappa \leq|\Omega|$. If $U$ contains an injective $f$ and every $a \in \operatorname{Sym}(\Omega)$ with $\operatorname{supp}(a) \subseteq \Omega f$ and $|\operatorname{supp}(a)|<\kappa$, then there exists an injective $f^{*} \in \mathcal{C}(U, \Lambda)$ such that $d\left(f^{*}\right) \geq \kappa$ and $\Omega f^{*} \subseteq \Omega f$.

Proof. We prove by transfinite induction that for each cardinal $\mu \leq \kappa$, (2)

there exists $f_{\mu} \in \mathcal{C}(U, \Lambda)$ such that $f_{\mu}$ is injective, $d\left(f_{\mu}\right) \geq \mu$, and $\Omega f_{\mu} \subseteq \Omega f$.

If there exists $f_{\mu} \in \mathcal{C}(U, \Lambda)$ such that $f_{\mu}$ is injective and $d\left(f_{\mu}\right) \geq \mu$, then, since $f_{\mu}$ and $f$ are injective, $f_{\mu} f \in \mathcal{C}(U, \Lambda), f_{\mu} f$ is injective, $d\left(f_{\mu} f\right)=d\left(f_{\mu}\right)+d(f) \geq \mu$ and $\Omega f_{\mu} \subseteq \Omega f$; i.e. (2) holds for $f_{\mu} f$. Hence it suffices to show that there exists $f_{\mu} \in \mathcal{C}(U, \Lambda)$ such that $f_{\mu}$ is injective and $d\left(f_{\mu}\right) \geq \mu$.

Since $U \nsubseteq S_{2}$, there exists an injective $h_{0} \in U \subseteq \mathcal{C}(U, \Lambda)$ such that $d\left(h_{0}\right)>0$. Since $h_{0}$ is injective, $h_{0}^{n}$ belongs to $\mathcal{C}(U, \Lambda)$ and, by Lemma 5.4(iii), $d\left(h_{0}^{n}\right) \geq n$ for all $n \in \mathbb{N}$. Thus (2) holds for all finite $\mu$.

Let $\mu$ be any cardinal such that $\aleph_{0} \leq \mu \leq \kappa$ and assume that (2) holds for every cardinal strictly less than $\mu$. Since $U \nsubseteq S_{4}(\mu)$, there exists $h_{1} \in U$ such that $c\left(h_{1}\right)<\mu \leq d\left(h_{1}\right)$. By our inductive hypothesis, there exists an injective $f_{c\left(h_{1}\right)} \in \mathcal{C}(U, \Lambda)$ such that $d\left(f_{c\left(h_{1}\right)}\right) \geq c\left(h_{1}\right)$ and $\Omega f_{c\left(h_{1}\right)} \subseteq \Omega f$. Since $f_{c\left(h_{1}\right)}$ is injective, $\left|\left(\Omega \backslash \Omega f_{c\left(h_{1}\right)}\right) f_{c\left(h_{1}\right)}\right|=\left|\Omega \backslash \Omega f_{c\left(h_{1}\right)}\right|$ and so

$$
\left|\Omega \backslash \Omega f_{c\left(h_{1}\right)}\right|=\left|\left(\Omega \backslash \Omega f_{c\left(h_{1}\right)}\right) f_{c\left(h_{1}\right)}\right|=\left|\Omega f_{c\left(h_{1}\right)} \backslash \Omega f_{c\left(h_{1}\right)}^{2}\right| \leq\left|\Omega f \backslash \Omega f_{c\left(h_{1}\right)}^{2}\right| .
$$

It follows that

$\left|\Omega f_{c\left(h_{1}\right)}^{2} \backslash \Lambda\left(h_{1}\right)\right| \leq\left|\Omega \backslash \Lambda\left(h_{1}\right)\right|=c\left(h_{1}\right) \leq d\left(f_{c\left(h_{1}\right)}\right)=\left|\Omega \backslash \Omega f_{c\left(h_{1}\right)}\right| \leq\left|\Omega f \backslash \Omega f_{c\left(h_{1}\right)}^{2}\right|$.

Thus there is $a \in \operatorname{Sym}(\Omega)$ such that

$$
\left(\Omega f_{c\left(h_{1}\right)}^{2} \backslash \Lambda\left(h_{1}\right)\right) a \subseteq \Omega f \backslash \Omega f_{c\left(h_{1}\right)}^{2}
$$

and $\operatorname{supp}(a) \subseteq\left(\Omega f_{c\left(h_{1}\right)}^{2} \backslash \Lambda\left(h_{1}\right)\right) \cup\left(\Omega f_{c\left(h_{1}\right)}^{2} \backslash \Lambda\left(h_{1}\right)\right) a$; see Figure 2. Hence, since $\Omega f_{c\left(h_{1}\right)} \subseteq \Omega f$, it follows that $\operatorname{supp}(a) \subseteq \Omega f$ and

$$
|\operatorname{supp}(a)| \leq 2\left|\Omega f_{c\left(h_{1}\right)}^{2} \backslash \Lambda\left(h_{1}\right)\right| \leq 2\left|\Omega \backslash \Lambda\left(h_{1}\right)\right|<\mu \leq \kappa .
$$

In particular, $a, a^{-1} \in U$.

From the definition of $a$, it follows that

$$
\Omega f_{c\left(h_{1}\right)}^{2} \backslash \Lambda\left(h_{1}\right) \subseteq\left(\Omega f \backslash \Omega f_{c\left(h_{1}\right)}^{2}\right) a^{-1}
$$




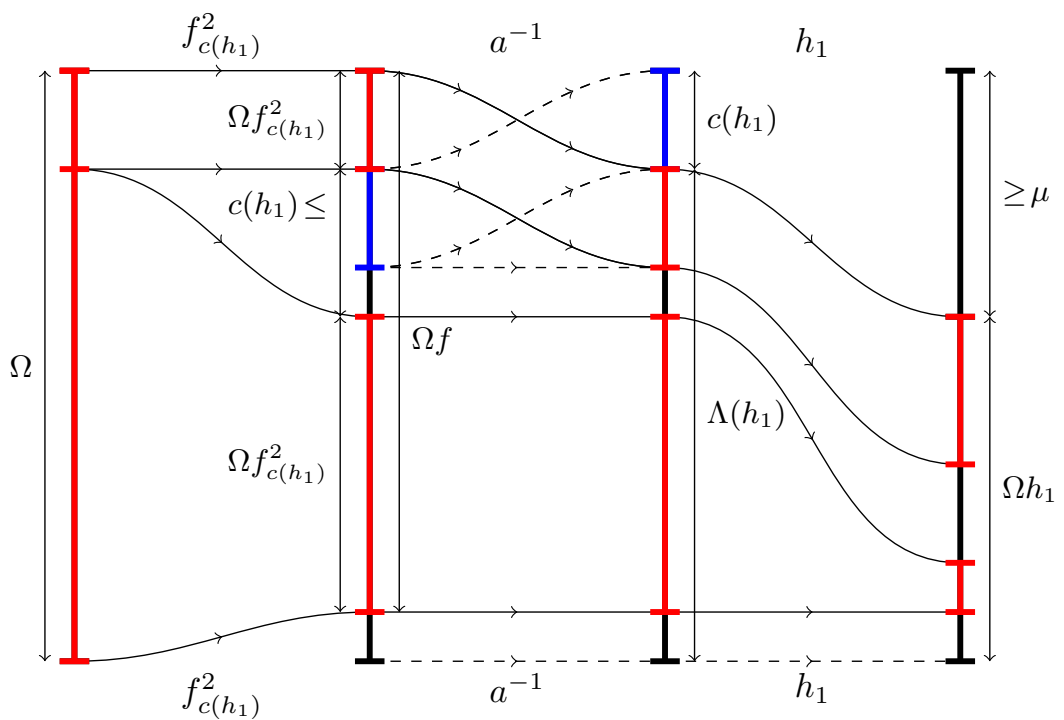

Figure 2. The composition $f_{c\left(h_{1}\right)}^{2} a^{-1} h_{1}$ from the proof of Lemma 6.3

and so $\Omega f_{c\left(h_{1}\right)}^{2} a^{-1} \subseteq \Lambda\left(h_{1}\right)$; see Figure 2 This shows that $f_{c\left(h_{1}\right)}^{2} a^{-1} h_{1} \in \mathcal{C}(U, \Lambda)$ is injective, and $d\left(f_{c\left(h_{1}\right)}^{2} a^{-1} h_{1}\right) \geq d\left(h_{1}\right) \geq \mu$ by Lemma [5.4(ii). It follows that $f_{\mu}:=f_{c\left(h_{1}\right)}^{2} a^{-1} h_{1} \in \mathcal{C}(U, \Lambda)$, and so (2) holds for $\mu$.

Lemma 6.4. Let $U$ be a subset of $\Omega^{\Omega}$ which is not contained in $S_{1}$ or $S_{3}(\mu)$ for any infinite $\mu \leq|\Omega|$, and let $\kappa$ be any cardinal such that $\aleph_{0} \leq \kappa \leq|\Omega|$. If there exist a surjective $g \in U$ and a transversal $\Gamma$ of $g$ such that $U$ contains every $a \in \operatorname{Sym}(\Omega)$ with $\operatorname{supp}(a) \subseteq \Gamma$ and $|\operatorname{supp}(a)|<\kappa$, then there exists $g^{*} \in\langle U\rangle$ such that $c\left(g^{*}\right) \geq \kappa$ and $\Gamma g^{*}=\Omega$.

Proof. Let $g^{\prime} \in \Omega^{\Omega}$ be any inverse for $g$ such that $\Omega g^{\prime}=\Gamma$ and let $u^{\prime} \in \Omega^{\Omega}$ be an arbitrary inverse for $u$ for all $u \in U \backslash\{g\}$. We denote $\left\{u^{\prime} \in \Omega^{\Omega}: u \in U\right\}$ by $U^{\prime}$ and we set $\Lambda: U^{\prime} \rightarrow \mathcal{P}(\Omega)$ to be the assignment of transversals for $U^{\prime}$ defined by $\Lambda\left(u^{\prime}\right)=\Omega u$. Recall that $c(u)=d\left(u^{\prime}\right)$ and $d(u)=c\left(u^{\prime}\right)$ for all $u \in U$.

We prove that $U^{\prime}, g^{\prime}$, and $\Lambda$ satisfy the conditions of Lemma 6.3. Since $U \nsubseteq$ $S_{1}, U \nsubseteq S_{3}(\mu)$, it follows that $U^{\prime} \nsubseteq S_{2}$ and $U^{\prime} \nsubseteq S_{4}(\mu)$ for all infinite $\mu \leq \kappa$. Since $g$ is surjective, $g^{\prime}$ is injective and by assumption $\Omega g^{\prime}=\Gamma$. In particular, $U^{\prime}$ contains every $a^{\prime}=a^{-1} \in \operatorname{Sym}(\Omega)$ where $\operatorname{supp}(a) \subseteq \Omega g^{\prime}$ and $|\operatorname{supp}(a)| \leq \kappa$. Thus by Lemma 6.3 there exists an injective $f^{*} \in \mathcal{C}\left(U^{\prime}, \Lambda\right)$ such that $d\left(f^{*}\right) \geq \kappa$ and $\Omega f^{*} \subseteq \Omega g^{\prime}$. By Corollary 5.3. $\langle U\rangle$ contains an inverse $g^{*}$ of $f^{*}$. Therefore $c\left(g^{*}\right)=d\left(f^{*}\right) \geq \kappa$ and $\Omega f^{*} \subseteq \Gamma$ is a transversal of $g^{*}$, and in particular $\Gamma g^{*}=\Omega$.

Proof of Theorem A. Let $M$ be a subsemigroup of $\Omega^{\Omega}$ containing $\operatorname{Sym}(\Omega)$. We first prove that if $M$ is not contained in any of $S_{1}, S_{2}, S_{3}(\mu), S_{4}(\mu)$, or $S_{5}$ where $\mu$ is any infinite cardinal not greater than $|\Omega|$, then $M=\Omega^{\Omega}$. By Lemmas 6.3 and 6.4, there exist $f, g \in M$ such that $f$ is injective, $d(f)=|\Omega|, g$ is surjective, and $c(g)=|\Omega|$. By Theorem 6.1, it suffices to show that there exists a surjective $h \in M$ such that 


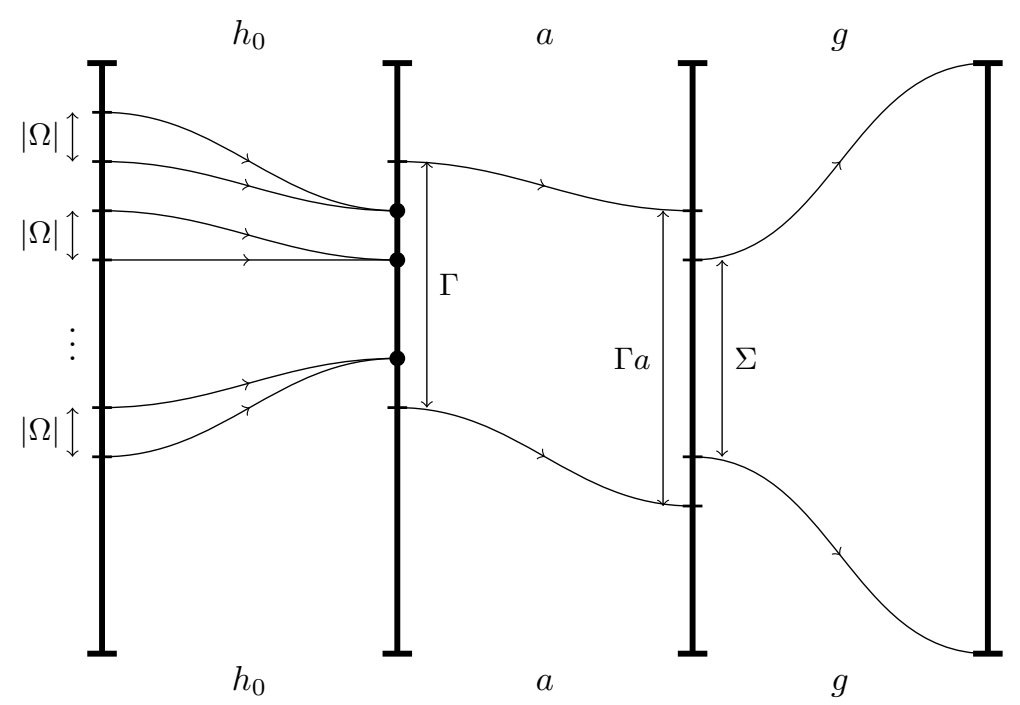

Figure 3. The composite $h_{0} a g$ in the proof of Theorem $\mathrm{A}$.

$k(h,|\Omega|)=|\Omega|$. Since $M \nsubseteq S_{5}$, there exists $h_{0} \in M$ such that $k\left(h_{0},|\Omega|\right)=|\Omega|$. Let $\Gamma=\left\{\alpha \in \Omega:\left|\alpha h_{0}^{-1}\right|=|\Omega|\right\}$. Then $|\Gamma|=|\Omega|$. Let $a \in \operatorname{Sym}(\Omega)$ be any element such that $\Gamma a$ contains a transversal $\Sigma$ of $g$. So, if $\alpha \in \Omega$, then there exists $\beta \in \Sigma$ such that $\beta g=\alpha$ and so $\alpha\left(h_{0} a g\right)^{-1}=\alpha g^{-1} a^{-1} h_{0}^{-1} \supseteq \beta a^{-1} h_{0}^{-1}$. But $\beta a^{-1} \in \Gamma$ and so $\left|\beta a^{-1} h_{0}^{-1}\right|=|\Omega|$. Thus $\left|\alpha\left(h_{0} a g\right)^{-1}\right|=|\Omega|$ and, since $\alpha \in \Omega$ was arbitrary, it follows that $h_{0} a g$ is surjective and $k\left(h_{0} a g,|\Omega|\right)=|\Omega|$. So the proof is concluded by setting $h=h_{0}$ ag; see Figure 3 .

If $|\Omega|$ is regular, then from the above either $M$ is contained in one of $S_{1}, S_{2}, S_{3}(\mu)$, $S_{4}(\mu)$, or $S_{5}$ or $M=\Omega^{\Omega}$. It then follows that if $M$ is a maximal subsemigroup of $\Omega^{\Omega}$ containing $\operatorname{Sym}(\Omega)$, then $M$ equals one of $S_{1}, S_{2}, S_{3}(\mu), S_{4}(\mu)$, or $S_{5}$. On the other hand, if $M$ is one of the semigroups $S_{1}, S_{2}, S_{3}(\mu), S_{4}(\mu)$, or $S_{5}$, then, since none of these semigroups are contained in any other, it follows that $M$ is a maximal subsemigroup of $\Omega^{\Omega}$.

Suppose that $|\Omega|$ is singular. If $M$ is not contained in any of the semigroups $S_{1}$, $S_{2}, S_{3}(\mu), S_{4}(\mu)$, or $S_{5}^{\prime}$, then, by Lemma 6.2. $M$ is also not contained in $S_{5}$ and so, from the above, $M=\Omega^{\Omega}$. Hence as in the case that $|\Omega|$ is regular, it follows that $M$ is a maximal subsemigroup of $\Omega^{\Omega}$ if and only if $M$ equals one of $S_{1}, S_{2}, S_{3}(\mu)$, $S_{4}(\mu)$, or $S_{5}^{\prime}$.

\section{Pointwise stabilisers of finite sets - The proof of Theorem B}

In this section we prove Theorem $\mathrm{B}$.

Theorem B. Let $\Omega$ be any infinite set and let $\Sigma$ be a non-empty finite subset of $\Omega$. Then the maximal subsemigroups of $\Omega^{\Omega}$ containing the pointwise stabiliser $\operatorname{Sym}(\Omega)_{(\Sigma)}$ but not $\operatorname{Sym}(\Omega)$ are:

$$
\begin{aligned}
& F_{1}(\Gamma, \mu)=\left\{f \in \Omega^{\Omega}: d(f) \geq \mu \text { or } \Gamma \nsubseteq \Omega f \text { or }\left(\Gamma f^{-1} \subseteq \Gamma \text { and } c(f)<\mu\right)\right\} \cup \mathfrak{F}, \\
& F_{2}(\Gamma, \nu)=\left\{f \in \Omega^{\Omega}: c(f) \geq \nu \text { or }|\Gamma f|<|\Gamma| \text { or }(\Gamma f=\Gamma \text { and } d(f)<\nu)\right\} \cup \mathfrak{F},
\end{aligned}
$$


where $\Gamma$ is a non-empty subset of $\Sigma$ and $\mu$ and $\nu$ are infinite cardinals with $\mu \leq|\Omega|^{+}$ and either $|\Gamma|=1$ and $\nu=|\Omega|^{+}$or $|\Gamma| \geq 2$ and $\nu \leq|\Omega|^{+}$.

Throughout this section we let $\Sigma$ be a non-empty finite subset of $\Omega$. We start by showing that the sets given in the theorem are actually semigroups.

Proposition 7.1. Let $\mu$ be any infinite cardinal such that $\mu \leq|\Omega|^{+}$and let $\Gamma$ be any non-empty subset of $\Omega$. Then $F_{1}(\Gamma, \mu)$ and $F_{2}(\Gamma, \mu)$ as defined in Theorem $\mathrm{B}$ are subsemigroups of $\Omega^{\Omega}$.

Proof. Let $f, g \in F_{1}(\Gamma, \mu)$. If $f \in \mathfrak{F}$ or $g \in \mathfrak{F}$, then $f g \in \mathfrak{F}$. If $d(g) \geq \mu$, then Lemma 5.4(ii) implies that $d(f g) \geq d(g) \geq \mu$ and so $f g \in F_{1}(\Gamma, \mu)$. If $\Gamma \nsubseteq \Omega g$, then $\Gamma \nsubseteq \Omega f g$ and so $f g \in F_{1}(\Gamma, \mu)$. Assume that $\Gamma g^{-1} \subseteq \Gamma$ and $c(g)<\mu$. If $d(f) \geq \mu$, then, by Lemma 5.4(vi), $d(f g) \geq \mu$. If $\Gamma \nsubseteq \Omega f$, then either $\Gamma \nsubseteq \Omega g$ or $\Gamma \subseteq \Omega g$. In the former case, $\Gamma \nsubseteq \Omega f g$, and in the latter, $\Gamma g^{-1}=\Gamma \nsubseteq \Omega f$ and so $\Gamma \nsubseteq \Omega f g$. In either case, $f g \in F_{1}(\Gamma, \mu)$. If $\Gamma f^{-1} \subseteq \Gamma$ and $c(f)<\mu$, then $\Gamma(f g)^{-1} \subseteq \Gamma$ and $c(f g) \leq c(f)+c(g)<\mu$ by Lemma 5.4(iv). Hence $F_{1}(\Gamma, \mu)$ is a semigroup.

Let $f, g \in F_{2}(\Gamma, \mu)$. If $f \in \mathfrak{F}$ or $g \in \mathfrak{F}$, then $f g \in \mathfrak{F}$. If $c(f) \geq \mu$, then $c(f g) \geq$ $c(f) \geq \mu$ by Lemma[5.4(iv) and so $f g \in F_{2}(\Gamma, \mu)$. If $|\Gamma f|<|\Gamma|$, then $|\Gamma f g|<|\Gamma|$ and so $f g \in F_{2}(\Gamma, \mu)$. Hence we may assume that $\Gamma f=\Gamma$ and $d(f)<\mu$. If $c(g) \geq \mu$, then, by Lemma 5.4(vii), $c(f g) \geq \mu$ and so $f g \in F_{2}(\Gamma, \mu)$. If $|\Gamma g|<|\Gamma|$, then $|\Gamma f g|=|\Gamma g|<|\Gamma|$ and $f g \in F_{2}(\Gamma, \mu)$. If $\Gamma g=\Gamma$ and $d(g)<\mu$, then $\Gamma f g=\Gamma g=\Gamma$ and $d(f g) \leq d(f)+d(g)<\mu$, by Lemma [5.4(ii), and so $f g \in F_{2}(\Gamma, \mu)$.

We require the following two lemmas to prove Theorem $B$.

Lemma 7.2. Let $\Sigma$ be a finite subset of $\Omega$ and let $U$ be a subset of $\Omega^{\Omega}$ containing $\operatorname{Sym}(\Omega)_{(\Sigma)}$ but which is not contained in $S_{2}$ or in $F_{2}(\Gamma, \mu)$ for any non-empty subset $\Gamma$ of $\Sigma$ and any infinite cardinal $\mu \leq|\Omega|^{+}$. If $\Lambda$ is an assignment of transversals (as defined in Definition [5.2) for $U$ such that $\Gamma \subseteq \Lambda(u)$ for all $u \in U \backslash F_{2}(\Gamma, \mu)$, then there exists an injective $f \in \mathcal{C}(U, \Lambda)$ such that $\Omega f \cap \Sigma=\emptyset$.

Proof. Since $\Sigma$ is finite (and $1_{\Omega} \in U$ ), it suffices to show that for every injective $f_{0} \in \mathcal{C}(U, \Lambda)$ with $\Omega f_{0} \cap \Sigma \neq \emptyset$ there exists an injective $f_{1} \in \mathcal{C}(U, \Lambda)$ with $\Omega f_{1} \cap \Sigma \subsetneq$ $\Omega f_{0} \cap \Sigma$. We will denote $\Omega f_{0} \cap \Sigma$ by $\Gamma$. We start by showing that there exists an injective $f_{2} \in \mathcal{C}(U, \Lambda)$ such that $\Omega f_{2} \cap \Sigma \subseteq \Gamma$ and $\Gamma f_{2} \neq \Gamma$.

If $\Gamma f_{0} \neq \Gamma$, then let $f_{2}=f_{0}$. Hence we may assume that $\Gamma f_{0}=\Gamma$. Since $U$ is not contained in $S_{2}$, there exists an injective $s \in U \backslash S_{2}$ such that $d(s)>0$. If $\Gamma s \neq \Gamma$, then we set $f_{2}=s f_{0}$. Thus the final case to consider is when $\Gamma s=\Gamma$.

For every infinite cardinal $\mu$ with $\mu \leq|\Omega|^{+}$, let $h_{\mu}$ be an element of $U \backslash F_{2}(\Gamma, \mu)$. Then the following hold:

$$
c\left(h_{\mu}\right)<\mu, \quad \Gamma \subseteq \Lambda\left(h_{\mu}\right)
$$

and either

$$
\Gamma h_{\mu} \neq \Gamma \text { or } d\left(h_{\mu}\right) \geq \mu \text {. }
$$

Note that $d\left(h_{|\Omega|^{+}}\right) \leq|\Omega|<|\Omega|^{+}$and so $\Gamma h_{|\Omega|^{+}} \neq \Gamma$. Thus we may let $\lambda$ be the least infinite cardinal such that $\Gamma h_{\lambda} \neq \Gamma$. We will show by transfinite induction that for every cardinal $\mu$ strictly less than $\lambda$

(3)

there exists an injective $g_{\mu} \in \mathcal{C}(U, \Lambda)$ with $\Omega g_{\mu} \cap \Sigma=\Gamma, \Gamma g_{\mu}=\Gamma$ and $d\left(g_{\mu}\right) \geq \mu$. 


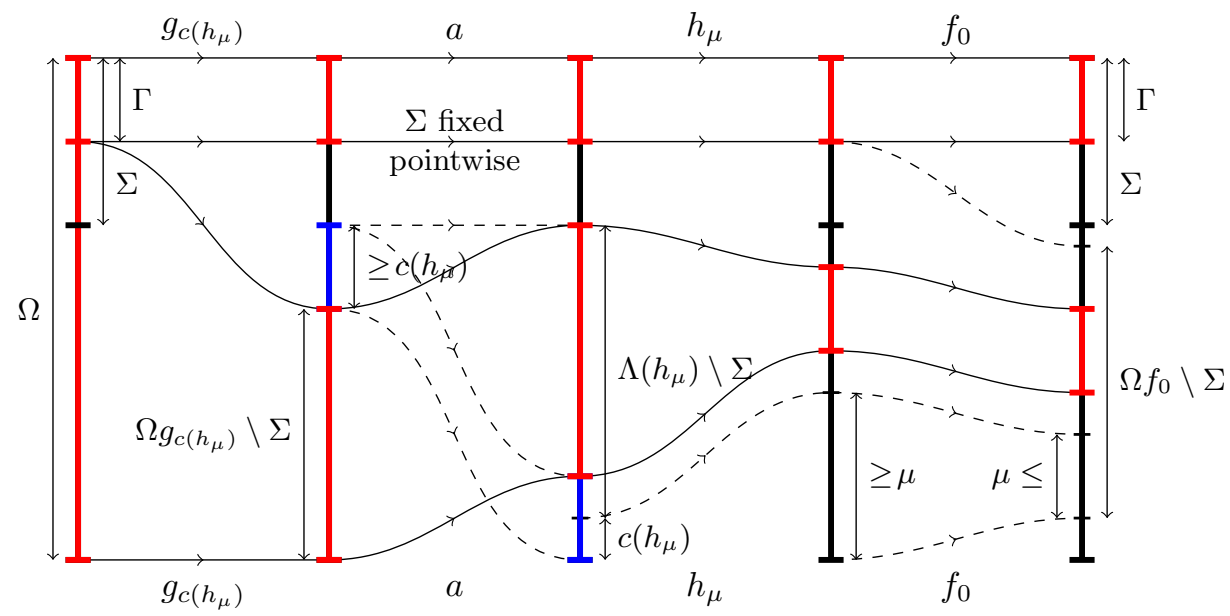

Figure 4. The composite $g_{c\left(h_{\mu}\right)} a h_{\mu} f_{0}$ from the proof of Lemma 7.2

For any finite $\mu$, we may let $g_{\mu}:=s^{\mu} f_{0}$. So let $\mu<\lambda$ be an infinite cardinal and assume (3) holds for all cardinals strictly less than $\mu$. By the inductive assumption there exists an injective $g_{c\left(h_{\mu}\right)} \in \mathcal{C}(U, \Lambda)$ with $\Omega g_{c\left(h_{\mu}\right)} \cap \Sigma=\Gamma, \Gamma g_{c\left(h_{\mu}\right)}=\Gamma$, and $d\left(g_{c\left(h_{\mu}\right)}\right) \geq c\left(h_{\mu}\right)$. Hence there exists a bijection $a \in \operatorname{Sym}(\Omega)_{(\Sigma)}$ such that $\left(\Omega g_{c\left(h_{\mu}\right)}\right) a \subseteq \Lambda\left(h_{\mu}\right)$. We define $g_{\mu}:=g_{c\left(h_{\mu}\right)} a h_{\mu} f_{0}$; see Figure 4. Then by construction $g_{\mu} \in \mathcal{C}(U, \Lambda), g_{\mu}$ is injective, and since $f_{0}$ is injective $d\left(g_{\mu}\right) \geq d\left(h_{\mu}\right) \geq \mu$. Also $\Gamma h_{\mu}=\Gamma$, since $\mu<\lambda$, and $g_{c\left(h_{\mu}\right)}, f, a$ stabilise $\Gamma$ setwise, and hence $\Gamma g_{\mu}=\Gamma$. Finally,

$$
\Gamma=\Gamma g_{\mu}=\Gamma g_{\mu} \cap \Sigma \subseteq \Omega g_{\mu} \cap \Sigma \subseteq \Omega f_{0} \cap \Sigma=\Gamma .
$$

Hence (3) holds for all $\mu<\lambda$.

Since $c\left(h_{\lambda}\right)<\lambda$, there exists an injective $g_{c\left(h_{\lambda}\right)} \in \mathcal{C}(U, \Lambda)$ with $\Omega g_{c\left(h_{\lambda}\right)} \cap \Sigma=\Gamma$, $\Gamma g_{c\left(h_{\lambda}\right)}=\Gamma$ and $d\left(g_{c\left(h_{\lambda}\right)}\right) \geq c\left(h_{\lambda}\right)$. Let $b \in \operatorname{Sym}(\Omega)_{(\Sigma)}$ be such that $\left(\Omega g_{c\left(h_{\lambda}\right)}\right) b \subseteq$ $\Lambda\left(h_{\lambda}\right)$; see Figure 5. Then $g_{c\left(h_{\lambda}\right)} b h_{\lambda}$ is injective, and since $g_{c\left(h_{\lambda}\right)}$ and $b$ stabilise $\Gamma$ setwise but $h_{\lambda}$ does not, $\Gamma g_{c\left(h_{\lambda}\right)} b h_{\lambda} \neq \Gamma$. Thus we let $f_{2}=g_{c\left(h_{\lambda}\right)} a h_{\lambda}$, which completes this part of the proof.

We will use the function $f_{2}$ to prove that there exists an injective $f_{1} \in \mathcal{C}(U, \Lambda)$ with $\Omega f_{1} \cap \Sigma \subsetneq \Gamma$. If $\Omega f_{2} \cap \Sigma \neq \Gamma$, then setting $f_{1}=f_{2}$ concludes the proof. Hence we only have to consider the case when $\Omega f_{2} \cap \Sigma=\Gamma$. Since $\Gamma f_{2} \neq \Gamma$, it follows, in this case, that $\Gamma f_{2}^{-1} \not \subset \Gamma=\Omega f_{2} \cap \Sigma$. Thus there are two cases to consider: $\Gamma f_{2}^{-1} \nsubseteq \Omega f_{2}$ or $\Gamma f_{2}^{-1} \nsubseteq \Sigma$. If $\Gamma f_{2}^{-1} \nsubseteq \Omega f_{2}$, then $\Omega f_{2}^{2} \cap \Sigma \subsetneq \Gamma$, and we set $f_{1}=f_{2}^{2}$. If $\Gamma f_{2}^{-1} \nsubseteq \Sigma$, then there exists $i \in \Gamma f_{2}^{-1} \backslash \Sigma$. Since $U$ is not contained in $S_{2}$, there exists $s \in U \backslash S_{2}$ such that $s$ is injective and $d(s)>0$. It follows from Lemma 5.4(iii) that $d\left(s^{n}\right)>|\Sigma|$ for some $n \in \mathbb{N}$. Hence there exists $j \in \Omega \backslash \Sigma$ such that $j \notin \Omega s^{n}$ and there is $p \in \operatorname{Sym}(\Omega)_{(\Sigma)}$ such that $(j) p=i$. In this case, we set $f_{1}:=s^{n} p f_{2}$; see Figure 6. Then since $j \notin \Omega s^{n}$ and $p f_{2}$ is injective, it follows that $i f_{2}=(j) p f_{2} \notin \Omega s^{n} p f_{2}=\Omega f_{1}$. But $i f_{2} \in \Gamma$, and so $\Omega f_{1} \cap \Sigma \subseteq \Gamma \backslash\left\{i f_{2}\right\} \subsetneq \Gamma$, as required. 


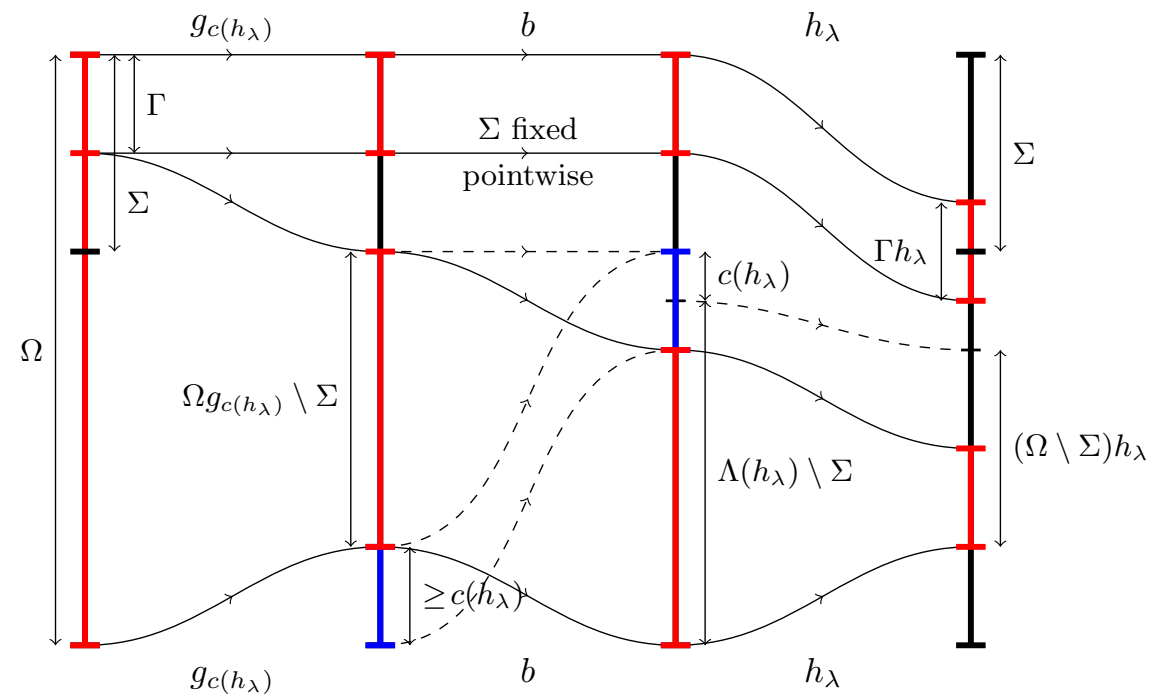

Figure 5. The composite $g_{c\left(h_{\lambda}\right)} b h_{\lambda}$ in the proof of Lemma 7.2

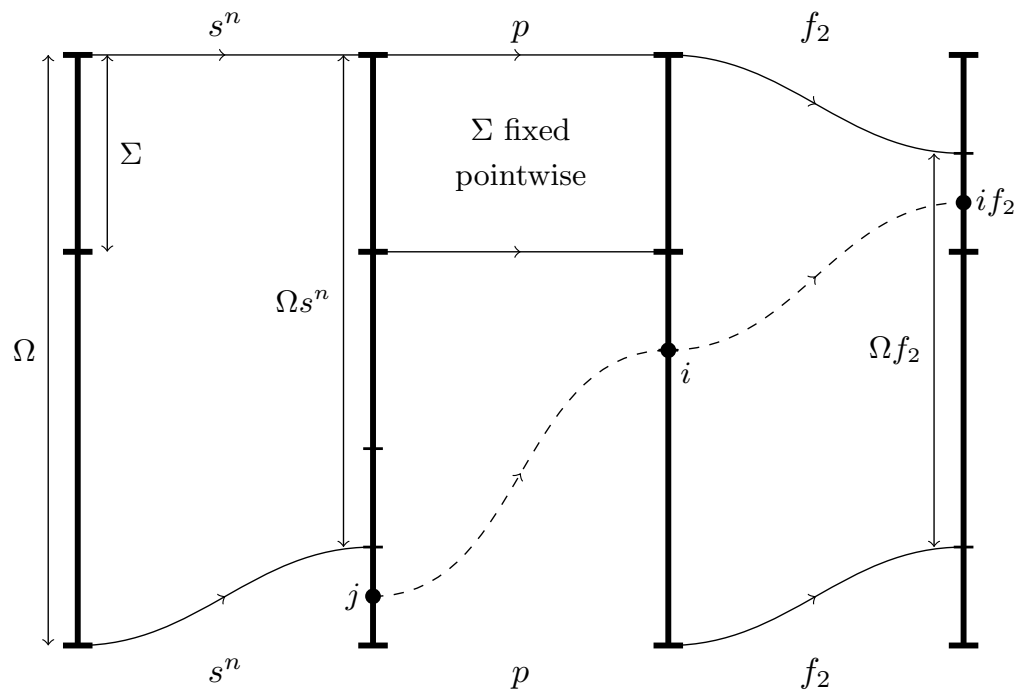

Figure 6. The composite $s^{n} p f_{2}$ in the proof of Lemma 7.2 .

Lemma 7.3. Let $\Sigma$ be a finite subset of $\Omega$ and let $U$ be a subset of $\Omega^{\Omega}$ containing $\operatorname{Sym}(\Omega)_{(\Sigma)}$ but which is not contained in $S_{1}$ or in $F_{1}(\Gamma, \mu)$ for any non-empty subset $\Gamma$ of $\Sigma$ and any infinite cardinal $\mu \leq|\Omega|^{+}$. Then there exists a surjective $g \in\langle U\rangle$ such that $(\Omega \backslash \Sigma) g=\Omega$.

Proof. If $u \in U$ is arbitrary, then we denote an arbitrary inverse for $u$ by $u^{\prime}$. We denote $\left\{u^{\prime} \in \Omega^{\Omega}: u \in U\right\}$ by $U^{\prime}$ and we set $\Lambda: U^{\prime} \rightarrow \mathcal{P}(\Omega)$ to be the assignment of transversals for $U^{\prime}$ defined by $\Lambda\left(u^{\prime}\right)=\Omega u$. 
Since $\operatorname{Sym}(\Omega)_{(\Sigma)} \subseteq U \nsubseteq S_{1} \cup F_{1}(\Gamma, \mu)$, it follows that $\operatorname{Sym}(\Omega)_{(\Sigma)} \subseteq U^{\prime} \nsubseteq S_{2} \cup$ $F_{2}(\Gamma, \mu)$ for all non-empty subsets $\Gamma$ of $\Sigma$ and for all infinite $\mu \leq|\Omega|^{+}$. If $u^{\prime} \notin$ $F_{2}(\Gamma, \mu)$ for some $u \in U$, then $u \notin F_{1}(\Gamma, \mu)$ and so $\Gamma \subseteq \Omega u=\Lambda\left(u^{\prime}\right)$. Thus by Lemma 7.2 there exists an injective $f \in \mathcal{C}\left(U^{\prime}, \Lambda\right)$ such that $\Omega f \cap \Sigma=\emptyset$. Then, by Corollary [5.3, $f$ has an inverse $g \in\langle U\rangle$. Then $g$ is surjective and $\Omega f$ is a transversal of $g$. In particular, $(\Omega \backslash \Sigma) g=\Omega g=\Omega$, as required.

Proof of Theorem B. It is straightforward to verify that none of the semigroups listed in the statement of Theorem $\mathrm{B}$ are contained in any of the others from that list. Moreover, none of these semigroups are contained in any of the semigroups from Theorem A

Let $M$ be a subsemigroup of $\Omega^{\Omega}$ containing $\operatorname{Sym}(\Omega)_{(\Sigma)}$ that is not contained in any of the semigroups in Theorems $\mathrm{A}$ or $\mathrm{B}$. We will prove that $\operatorname{Sym}(\Omega)$ is a subsemigroup of $M$ and so Theorem $\AA$ implies that $M=\Omega^{\Omega}$.

Let $\Gamma$ be a finite subset of $\Omega$ and let $\mu$ be an infinite cardinal such that $\mu \leq|\Omega|^{+}$. If $u \in \Omega^{\Omega}$ but $u \notin F_{2}(\Gamma, \mu)$, then, in particular, $u$ is injective on $\Gamma$ and so there exists a transversal of $u$ containing $\Gamma$. In particular, there is an assignment of transversals $\Lambda$ for $M$ such that $\Gamma \subseteq \Lambda(u)$ for all $u \in M \backslash F_{2}(\Gamma, \mu)$. Hence by Lemma 7.2 , there exists an injective $f \in M$ such that $\Omega f \cap \Sigma=\emptyset$. Since $M$ contains all permutations with support contained in $\Omega \backslash \Sigma$, it contains all permutations with support contained in $\Omega f$. Thus by Lemma 6.3 there exists an injective $f^{*} \in M$ with $d\left(f^{*}\right)=|\Omega|$ and $\Omega f^{*} \subseteq \Omega f \subseteq \Omega \backslash \Sigma$.

By Lemma 17.3 , there exists a surjective $g \in M$ with a transversal $\Gamma \subseteq \Omega \backslash \Sigma$. Clearly $M$ contains every permutation with support contained in $\Gamma$. Hence by Lemma 6.4 there exists $g^{*} \in M$ such that $c\left(g^{*}\right)=|\Omega|$ and $\Gamma g^{*}=\Omega$.

Since $\Omega f^{*}$ and $\Gamma$ are moieties of $\Omega \backslash \Sigma$, every bijection from $\Omega f^{*}$ to $\Gamma$ is a restriction of some element of $\operatorname{Sym}(\Omega)_{(\Sigma)}$. So, if $a \in \operatorname{Sym}(\Omega)$ is arbitrary, then, since $f^{*}$ and $\left.g^{*}\right|_{\Gamma}$ are injective, there exists $b \in \operatorname{Sym}(\Omega)_{(\Sigma)}$ such that $a=f^{*} b g^{*}$. Therefore $\operatorname{Sym}(\Omega)$ is a subsemigroup of $M$ and so, by Theorem $\mathrm{A}, M=\Omega^{\Omega}$.

We have shown that if $M$ is a subsemigroup of $\Omega^{\Omega}$ that contains $\operatorname{Sym}(\Omega)_{(\Sigma)}$, then either $M$ is contained in one of the semigroups from Theorem $\mathrm{A}$, one of the semigroups $F_{1}(\Gamma, \mu)$ or $F_{2}(\Gamma, \nu)$ from the statement of the theorem, or $M=\Omega^{\Omega}$. It follows that if $M$ is maximal, then $M$ is one of these semigroups. On the other hand, if $M$ is one of $F_{1}(\Gamma, \mu)$ or $F_{2}(\Gamma, \nu)$, then, since none of these semigroups are contained in any of the others or any of the semigroups in Theorem A, it follows that $M$ is a maximal subsemigroup of $\Omega^{\Omega}$.

\section{The stabiliser of AN ultrafilter - The proof of Theorem C}

In this section we give the proof of Theorem $\mathrm{C}$

Theorem C. Let $\Omega$ be any infinite set, let $\mathcal{F}$ be a non-principal ultrafilter on $\Omega$, and let $\kappa\left(\geq \aleph_{0}\right)$ be the least cardinality of an element of $\mathcal{F}$. Then the maximal subsemigroups of $\Omega^{\Omega}$ containing $\operatorname{Sym}(\Omega)_{\{\mathcal{F}\}}$ but not $\operatorname{Sym}(\Omega)$ are:

$$
\begin{aligned}
& U_{1}(\mathcal{F}, \mu)=\left\{f \in \Omega^{\Omega}:(d(f) \geq \mu) \text { or }(\Omega f \notin \mathcal{F})\right. \\
& \text { or }(c(f)<\mu \text { and }(\forall \Sigma \notin \mathcal{F})(\Sigma f \notin \mathcal{F}))\} \cup \mathfrak{F}, \\
& U_{2}(\mathcal{F}, \mu)=\left\{f \in \Omega^{\Omega}:(c(f) \geq \mu) \text { or }(\forall \Sigma \in \mathcal{F})\left(c\left(\left.f\right|_{\Sigma}\right)>0\right)\right. \\
& \text { or }(d(f)<\mu \text { and }(\forall \Sigma \in \mathcal{F})(\Sigma f \in \mathcal{F}))\} \cup \mathfrak{F}
\end{aligned}
$$

for cardinals $\mu$ such that $\kappa<\mu \leq|\Omega|^{+}$. 
Throughout this section we let $\mathcal{F}$ be an arbitrary non-principal ultrafilter on $\Omega$ and let $\kappa$ be the least cardinality of a set belonging to $\mathcal{F}$. Since $\mathcal{F}$ is non-principal, it follows that $\kappa \geq \aleph_{0}$. A subset $S$ of $\operatorname{Sym}(\Omega)$ is transitive on moieties of $\Omega$ if for all moieties $\Sigma, \Gamma$ of $\Omega$ there exists $f \in S$ such that $\Sigma f=\Gamma$. Recall that $\operatorname{Sym}(\Omega)_{\{\mathcal{F}\}}$ is transitive on moieties in $\mathcal{F}$ and hence also on moieties not in $\mathcal{F}$. Moreover, if $\Gamma, \Sigma \in \mathcal{F}$ such that $|\Omega \backslash \Gamma| \geq|\Omega \backslash \Sigma|$, then there exists $a \in \operatorname{Sym}(\Omega)_{\{\mathcal{F}\}}$ such that $\Gamma a \subset \Sigma$.

The following lemma and its proof are similar to Lemma 7.2, We use the following observation in the statement and proof of the next lemma. If $f \in \Omega^{\Omega}$ but $f \notin$ $U_{2}(\mathcal{F}, \mu)$, then there exists $\Sigma \in \mathcal{F}$ such that $c\left(\left.f\right|_{\Sigma}\right)=0$; in other words $f$ is injective on $\Sigma$. It follows that $\Sigma$ is contained in a transversal $\Lambda(f)$ for $f$ and so $\Lambda(f) \in \mathcal{F}$. We have shown that every element of $\Omega^{\Omega}$ which does not belong to $U_{2}(\mathcal{F}, \mu)$ has a transversal in $\mathcal{F}$.

Lemma 8.1. Let $U$ be a subset of $\Omega^{\Omega}$ containing the stabiliser $\operatorname{Sym}(\Omega)_{\{\mathcal{F}\}}$ of $\mathcal{F}$ but which is not contained in $U_{2}(\mathcal{F}, \mu), S_{2}$, or $S_{4}(\nu)$ for any cardinals $\mu, \nu$ such that $\aleph_{0} \leq \nu \leq \kappa<\mu \leq|\Omega|^{+}$, and let $\Lambda$ be an assignment of transversals (as defined in Definition 5.2) for $U$ such that $\Lambda(u) \in \mathcal{F}$ for all $u \in U \backslash U_{2}(\mathcal{F}, \mu)$. Then there exists an injective $f \in \mathcal{C}(U, \Lambda)$ such that $\Omega f \notin \mathcal{F}$.

Proof. If $\Sigma \subseteq \Omega$ such that $|\Sigma|<\kappa$, then $\Sigma \notin \mathcal{F}$ and so every $a \in \operatorname{Sym}(\Omega)$ such that $|\operatorname{supp}(a)|<\kappa$ belongs to $\operatorname{Sym}(\Omega)_{\{\mathcal{F}\}}$ and hence to $U$. Thus by Lemma 6.3 there exists an injective $f_{0} \in \mathcal{C}(U, \Lambda)$ such that $d\left(f_{0}\right) \geq \kappa$. We start by showing that there exists an injective $f_{1} \in \mathcal{C}(U, \Lambda)$ and $\Sigma \in \mathcal{F}$ such that $\Sigma f_{1} \notin \mathcal{F}$.

If there exists $\Sigma \in \mathcal{F}$ such that $\Sigma f_{0} \notin \mathcal{F}$, then $f_{1}:=f_{0}$ is the required function. Hence we may assume that $\Sigma f_{0} \in \mathcal{F}$ for all $\Sigma \in \mathcal{F}$. For every cardinal $\mu$ such that $\kappa<\mu \leq|\Omega|^{+}$, let $h_{\mu}$ be an element of $U \backslash U_{2}(\mathcal{F}, \mu)$. Then the following hold: $c\left(h_{\mu}\right)<\mu, \bar{\Lambda}\left(h_{\mu}\right) \in \mathcal{F}$, and either $d\left(h_{\mu}\right) \geq \mu$ or $\Sigma h_{\mu} \notin \mathcal{F}$ for some $\Sigma \in \mathcal{F}$. Note that $d\left(h_{|\Omega|^{+}}\right) \leq|\Omega|<|\Omega|^{+}$and so there exists $\Sigma \in \mathcal{F}$ such that $\Sigma h_{|\Omega|^{+}} \notin \mathcal{F}$. Thus we may define

$$
\lambda=\min \left\{\mu: \kappa<\mu \leq|\Omega|^{+} \text {and }(\exists \Sigma \in \mathcal{F})\left(\Sigma h_{\mu} \notin \mathcal{F}\right)\right\} .
$$

We will show, by transfinite induction, that for every cardinal $\mu$ strictly less than $\lambda$ :

$$
\text { there exists an injective } g_{\mu} \in \mathcal{C}(U, \Lambda)
$$

such that $d\left(g_{\mu}\right) \geq \mu$ and $\Sigma g_{\mu} \in \mathcal{F}$ for all $\Sigma \in \mathcal{F}$.

By assumption, $f_{0}$ satisfies (4) for all $\mu \leq \kappa$. So let $\mu$ be any cardinal such that $\kappa<\mu<\lambda$ and assume that (4) holds for all cardinals strictly less than $\mu$.

By the inductive assumption there exists an injective $g_{c\left(h_{\mu}\right)} \in \mathcal{C}(U, \Lambda)$ such that $d\left(g_{c\left(h_{\mu}\right)}\right) \geq c\left(h_{\mu}\right)$ and $\Sigma g_{c\left(h_{\mu}\right)} \in \mathcal{F}$ for all $\Sigma \in \mathcal{F}$. In particular, $\Omega g_{c\left(h_{\mu}\right)} \in \mathcal{F}$, and so by the comments preceding the lemma there exists $a \in \operatorname{Sym}(\Omega)_{\{\mathcal{F}\}}$ such that $\Omega g_{c\left(h_{\mu}\right)} a \subseteq \Lambda\left(h_{\mu}\right)$; see Figure 7, We define $g_{\mu}:=g_{c\left(h_{\mu}\right)} a h_{\mu}$. Then by construction $g_{\mu} \in \mathcal{C}(U, \Lambda), g_{\mu}$ is injective, and $d\left(g_{\mu}\right) \geq d\left(h_{\mu}\right) \geq \mu$. Also $\Sigma g_{\mu} \in \mathcal{F}$ for all $\Sigma \in \mathcal{F}$ since this property holds for $g_{c\left(h_{\mu}\right)}, a$, and $h_{\mu}$ (since $\mu<\lambda$ ). Hence (4) holds for all $\mu<\lambda$.

Since $c\left(h_{\lambda}\right)<\lambda$, there exists an injective $g_{c\left(h_{\lambda}\right)} \in \mathcal{C}(U, \Lambda)$ such that $d\left(g_{c\left(h_{\lambda}\right)}\right) \geq$ $c\left(h_{\lambda}\right)$ and $\Sigma g_{c\left(h_{\lambda}\right)} \in \mathcal{F}$ for all $\Sigma \in \mathcal{F}$. Then as above there exists $b \in \operatorname{Sym}(\Omega)_{\{\mathcal{F}\}}$ such that $\left(\Omega g_{c\left(h_{\lambda}\right)}\right) b \subseteq \Lambda\left(h_{\lambda}\right)$; see Figure 8 . Then $g_{c\left(h_{\lambda}\right)} b h_{\lambda} \in \mathcal{C}(U, \Lambda)$ is injective. By the definition of $\lambda$ there exists $\Sigma \in \mathcal{F}$ such that $\Sigma h_{\lambda} \notin \mathcal{F}$. Hence $\Sigma a^{-1} \in \mathcal{F}$ 


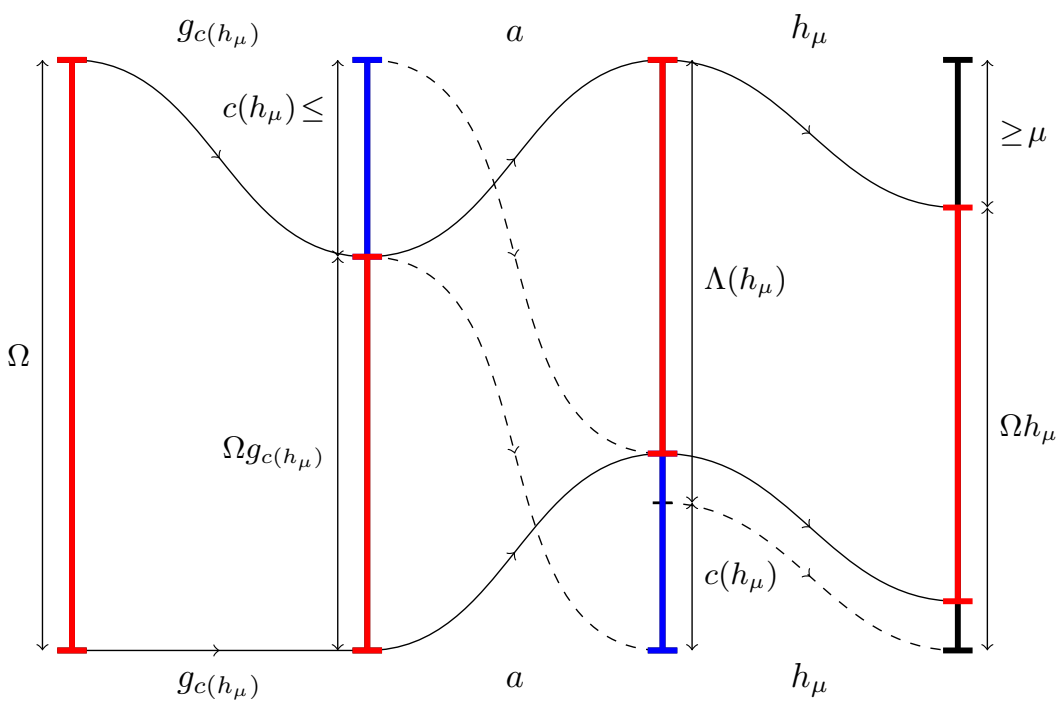

FiguRE 7. The composite $g_{c\left(h_{\mu}\right)} a h_{\mu}$ in the proof of Lemma 8.1

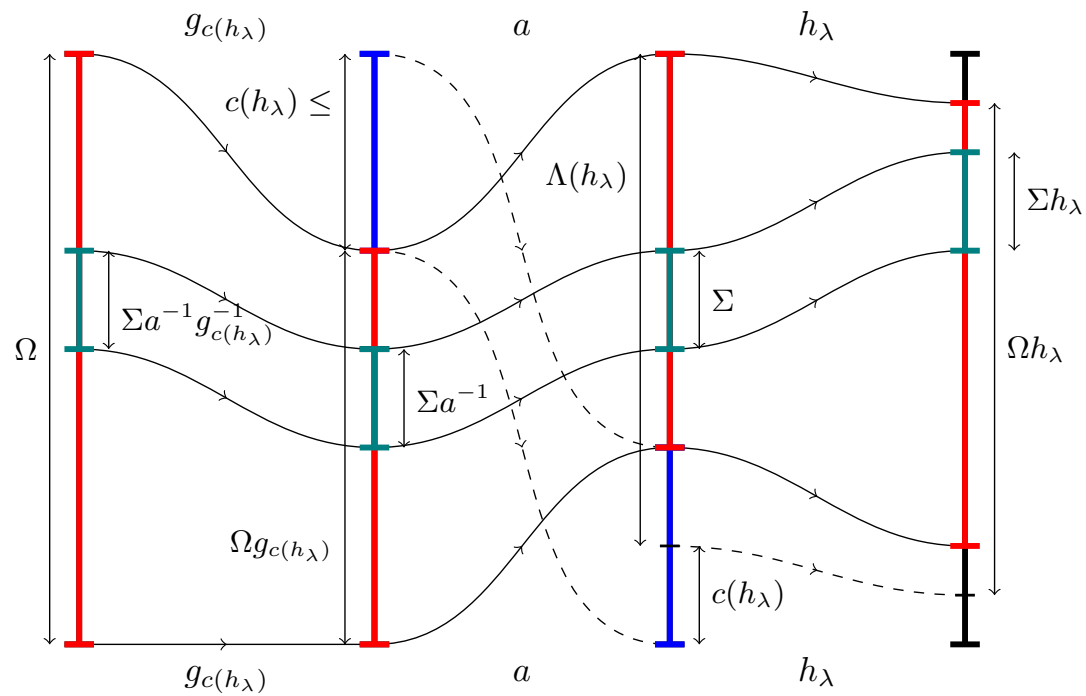

FIgURE 8. The composite $g_{c\left(h_{\lambda}\right)} a h_{\lambda}$ in the proof of Lemma 8.1 .

and so $\Omega \backslash \Sigma a^{-1} \notin \mathcal{F}$. It follows that $\left(\Omega \backslash \Sigma a^{-1}\right) g^{-1} \notin \mathcal{F}$, and, since $g$ is injective, we have that $\Sigma a^{-1} g^{-1} \in \mathcal{F}$. Thus if we let $f_{1}=g_{c\left(h_{\lambda}\right)} a h_{\lambda}$ and $\Sigma^{\prime}=\Sigma a^{-1} g^{-1} \in \mathcal{F}$, then $\Sigma^{\prime} f_{1}=\Sigma h_{\lambda} \notin \mathcal{F}$, which completes this part of the proof.

If $\Omega f_{1} \notin \mathcal{F}$, then $f_{1}$ satisfies the conclusion of the lemma. If $\Omega f_{1} \in \mathcal{F}$, then there exists $\Gamma \subseteq \Omega f_{1} \backslash \Sigma^{\prime} f_{1}$ such that $\Gamma \in \mathcal{F}$ and $|\Gamma|=\kappa$ (the least cardinality of a 


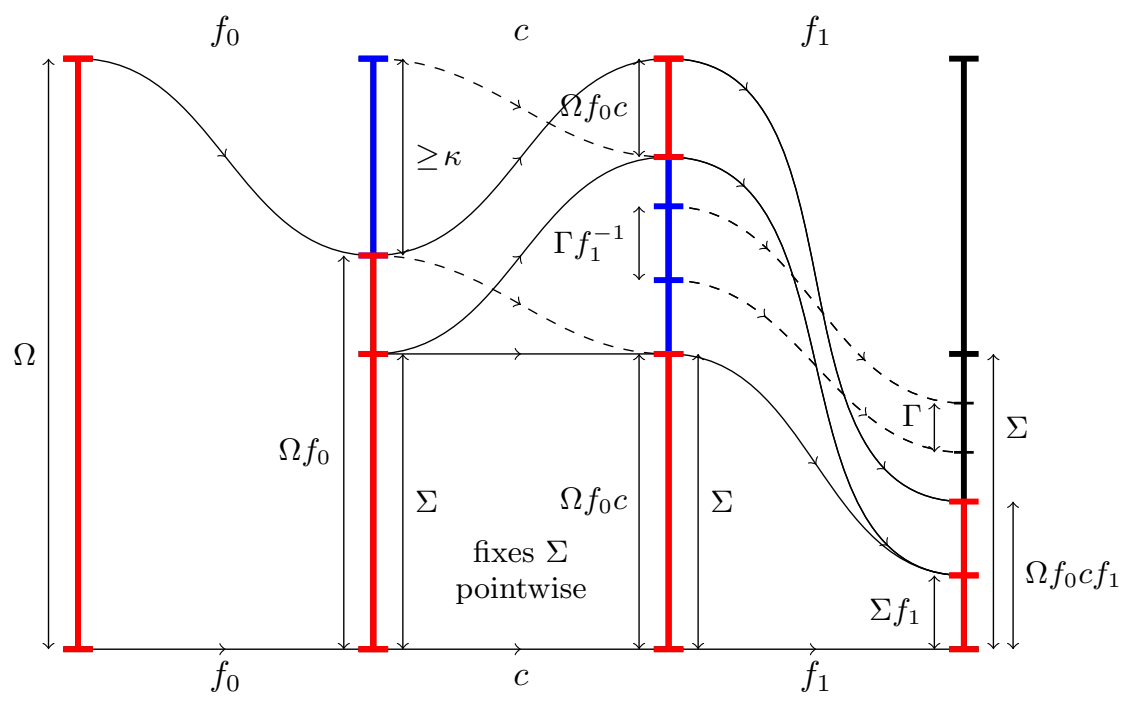

Figure 9. The composite $f_{0} c f_{1}$ in the proof of Lemma 8.1

set in $\mathcal{F})$. Hence $\Gamma f_{1}^{-1} \cap \Sigma^{\prime}=\emptyset$ and $\left|\Gamma f_{1}^{-1}\right|=\kappa$, since $f_{1}$ is injective. Thus there exists $c \in \operatorname{Sym}(\Omega)_{(\Sigma)} \leq \operatorname{Sym}(\Omega)_{\{\mathcal{F}\}}$ such that $\Gamma f_{1}^{-1} \subseteq \Omega \backslash \Omega f_{0} c$; see Figure 9] The required function is then $f=f_{0} c f_{1}$ since $\Omega f \subseteq \Omega \backslash \Gamma \notin \mathcal{F}$ and so $\Omega f \notin \mathcal{F}$. Finally, $f \in \mathcal{C}(U, \Lambda)$ since $f_{0}, a, f_{1} \in \mathcal{C}(U, \Lambda)$.

Lemma 8.2. Let $U$ be a subset of $\Omega^{\Omega}$ containing the stabiliser $\operatorname{Sym}(\Omega)_{\{\mathcal{F}\}}$ of $\mathcal{F}$ but which is not contained in $U_{1}(\mathcal{F}, \mu), S_{1}$, or $S_{3}(\nu)$ for any cardinals $\mu, \nu$ such that $\aleph_{0} \leq \nu \leq \kappa<\mu \leq|\Omega|^{+}$. Then there exists a surjective $g \in\langle U\rangle$ with a transversal $\Lambda(g)$ which does not belong to $\mathcal{F}$.

Proof. If $u \in U$ is arbitrary, then we denote an arbitrary inverse for $u$ by $u^{\prime}$. We denote $\left\{u^{\prime} \in \Omega^{\Omega}: u \in U\right\}$ by $U^{\prime}$ and we set $\Lambda: U^{\prime} \rightarrow \mathcal{P}(\Omega)$ to be the assignment of transversals for $U^{\prime}$ defined by $\Lambda\left(u^{\prime}\right)=\Omega u$.

Since $\operatorname{Sym}(\Omega)_{\{\mathcal{F}\}} \subseteq U \nsubseteq U_{2}(\mathcal{F}, \mu) \cup S_{1} \cup S_{3}(\nu)$, it follows that $\operatorname{Sym}(\Omega)_{\{\mathcal{F}\}} \subseteq$ $U^{\prime} \nsubseteq U_{2}(\mathcal{F}, \mu) \cup S_{2} \cup S_{4}(\nu)$ for any cardinals $\mu, \nu$ such that $\aleph_{0} \leq \nu \leq \kappa<\mu \leq|\Omega|^{+}$. If $u^{\prime} \notin U_{2}(\mathcal{F}, \mu)$ for some $u \in U$, then $u \notin U_{1}(\mathcal{F}, \mu)$ and so $\Lambda\left(u^{\prime}\right)=\Omega u \in \mathcal{F}$. Thus by Lemma 8.1 there exists an injective $f \in \mathcal{C}\left(U^{\prime}, \Lambda\right)$ such that $\Omega f \notin \mathcal{F}$. Then, by Corollary 5.3, $f$ has an inverse $g \in\langle U\rangle$. Then $g$ is surjective and $\Omega f \notin \mathcal{F}$ is a transversal of $g$, as required.

Proof of Theorem C. It is easy to check that $U_{1}(\mathcal{F}, \mu)$ and $U_{2}(\mathcal{F}, \mu)$ are semigroups and that neither is contained in the other nor in any of the semigroups listed in Theorem $\mathrm{A}$. Let $M$ be any subsemigroup of $\Omega^{\Omega}$ containing $\operatorname{Sym}(\Omega)_{\{\mathcal{F}\}}$. As in the proof of Theorem $\mathrm{B}$, it suffices to prove that if $M$ is not contained in any of the semigroups from Theorems $\mathrm{A}$ or $\mathrm{C}$. then $M=\Omega^{\Omega}$.

By Lemmas 8.1 and 8.2 , there exist $f, g \in M$ such that $f$ is injective, $\Omega f \notin \mathcal{F}$, $g$ is surjective and $g$ has a transversal $\Lambda(g) \notin \mathcal{F}$. Since $\operatorname{Sym}(\Omega)_{\{\mathcal{F}\}}$ contains the pointwise stabilisers in $\operatorname{Sym}(\Omega)$ of the complements of $\Omega f$ and $\Lambda(g)$, it follows from Lemmas 6.3 and 6.4 that there exist $f^{*}, g^{*} \in M$ with $f^{*}$ injective, $g^{*}$ surjective, 
$d\left(f^{*}\right)=c\left(g^{*}\right)=|\Omega|, \Omega f^{*} \subseteq \Omega f$ and a transversal $\Lambda\left(g^{*}\right) \subseteq \Lambda(g)$ for $g^{*}$. Also since $\Omega f, \Lambda(g) \notin \mathcal{F}$ it follows that $\Omega f^{*}, \Lambda\left(g^{*}\right) \notin \mathcal{F}$. Since $\operatorname{Sym}(\Omega)_{\{\mathcal{F}\}}$ is contained in $M$ and it is transitive on moieties not belonging to $\mathcal{F}$, it follows that every element of $\operatorname{Sym}(\Omega)$ can be given in the form $f^{*} a g^{*}$ for some $a \in \operatorname{Sym}(\Omega)_{\{\mathcal{F}\}}$. In particular, $\operatorname{Sym}(\Omega) \subseteq M$, and so, by Theorem $\mathrm{A}, M=\Omega^{\Omega}$.

\section{The Almost stabiliser of a Finite Partition - The Proof of Theorem D}

Recall that a finite partition of $\Omega$ is a partition of $\Omega$ into finitely many moieties. Throughout this section we denote the finite partition $\left\{\Sigma_{0}, \Sigma_{1}, \ldots, \Sigma_{n-1}\right\}$ of $\Omega$ with $n \geq 2$ by $\mathcal{P}$, and we write

$$
\operatorname{Stab}(\mathcal{P})=\left\{g \in \operatorname{Sym}(\Omega):(\forall i)(\exists j) \Sigma_{i} g=\Sigma_{j}\right\}
$$

for the stabiliser of $\mathcal{P}$.

A binary relation on an arbitrary set $\Lambda$ is just a subset of $\Lambda \times \Lambda$. If $\rho$ and $\sigma$ are binary relations on $\Lambda$, then the composition $\rho \sigma$ of $\rho$ and $\sigma$ is defined to be

$$
\rho \sigma=\{(\alpha, \beta) \in \Lambda \times \Lambda:(\exists \gamma)(\alpha, \gamma) \in \rho \text { and }(\gamma, \beta) \in \sigma\} .
$$

Composition of binary relations is associative, and so we may refer to the semigroup generated by a set of binary relations. A relation $\rho$ on $\Lambda$ is total if $\alpha \rho=\{\beta \in \Lambda$ : $(\alpha, \beta) \in \rho\} \neq \emptyset$ for all $\alpha \in \Lambda$.

Recall that if $f \in \Omega^{\Omega}$, then $\rho_{f}$ is the binary relation on $n=\{0,1, \ldots, n-1\}$ defined in (11) as

$$
\rho_{f}=\left\{(i, j):\left|\Sigma_{i} f \cap \Sigma_{j}\right|=|\Omega|\right\} .
$$

The purpose of this section is to prove the following theorem.

Theorem D. Let $\Omega$ be any infinite set and let $\mathcal{P}=\left\{\Sigma_{0}, \Sigma_{1}, \ldots, \Sigma_{n-1}\right\}, n \geq 2$, be a finite partition of $\Omega$. Then the maximal subsemigroups of $\Omega^{\Omega}$ containing $\operatorname{Stab}(\mathcal{P})$ but not $\operatorname{Sym}(\Omega)$ are:

$$
\begin{aligned}
& A_{1}(\mathcal{P})=\left\{f \in \Omega^{\Omega}: \rho_{f} \in \operatorname{Sym}(n) \text { or } \rho_{f} \text { is not total }\right\} \\
& A_{2}(\mathcal{P})=\left\{f \in \Omega^{\Omega}: \rho_{f} \in \operatorname{Sym}(n) \text { or } \rho_{f}^{-1} \text { is not total }\right\} .
\end{aligned}
$$

We start by showing that $A_{1}(\mathcal{P})$ and $A_{2}(\mathcal{P})$ in Theorem $\mathrm{D}$ are semigroups.

Proposition 9.1. The sets $A_{1}(\mathcal{P})$ and $A_{2}(\mathcal{P})$ as defined in Theorem $\mathrm{D}$ are subsemigroups of $\Omega^{\Omega}$, and neither is a subset of the other nor of any of the semigroups in Theorem $\mathrm{A}$.

Proof. It is easy to verify that neither $A_{1}(\mathcal{P})$ nor $A_{2}(\mathcal{P})$ is contained in the other nor in any of the semigroups listed in Theorem $\mathrm{A}$. We only prove that $A_{1}(\mathcal{P})$ is a subsemigroup of $\Omega^{\Omega}$; the proof that $A_{2}(\mathcal{P})$ is a subsemigroup follows by a dual argument.

Let $f, g \in A_{1}(\mathcal{P})$. Then, certainly, $\rho_{f g} \subseteq \rho_{f} \rho_{g}$. Hence, if $\rho_{f}$ is not total, then $\rho_{f} \rho_{g}$ is not total, and so $\rho_{f g}$ is not either, whence $f g \in A_{1}(\mathcal{P})$. Assume that $\rho_{f} \in \operatorname{Sym}(n)$. Then either $\rho_{f} \rho_{g} \in \operatorname{Sym}(n)$ or $\rho_{f} \rho_{g}$ is not total, depending on whether $\rho_{g} \in \operatorname{Sym}(n)$ or $\rho_{g}$ is not total. Hence $\rho_{f g} \in \operatorname{Sym}(n)$ or $\rho_{f g}$ is not total and in either case $f g \in A_{1}(\mathcal{P})$.

We prove Theorem $\mathrm{D}$ in a sequence of lemmas. If $\Sigma \subseteq \Omega$, then we denote by $\operatorname{Sym}(\Sigma)$ the pointwise stabiliser of $\Omega \backslash \Sigma$ in $\operatorname{Sym}(\Omega)$. 
Lemma 9.2. Let $f, g \in \Omega^{\Omega}$. Then there exists $a \in \operatorname{Stab}(\mathcal{P})$ such that $\rho_{\text {fag }}=\rho_{f} \rho_{g}$.

Proof. Let $i \in\{0,1, \ldots, n-1\}$ be arbitrary. If $j \in i \rho_{f}^{-1}$, then $\left|\Sigma_{j} f \cap \Sigma_{i}\right|=|\Omega|$, and so $\Sigma_{j} f \cap \Sigma_{i}$ can be partitioned into $\left|i \rho_{g}\right|+1$ moieties. If $k \in i \rho_{g}$, then $g$ has a transversal that intersects $\Sigma_{k} g^{-1} \cap \Sigma_{i}$ in a set $\Gamma_{k}$ where $\left|\Gamma_{k}\right|=|\Omega|$. Hence $\Gamma_{k}$ can be partitioned into $\left|i \rho_{f}^{-1}\right|+1$ moieties. Let $a_{i} \in \operatorname{Sym}\left(\Sigma_{i}\right)$ be any element mapping one of the moieties partitioning $\Sigma_{j} f \cap \Sigma_{i}$ to one of the moieties partitioning $\Gamma_{k}$ for all $j \in i \rho_{f}^{-1}$ and for all $k \in i \rho_{g}$. The required $a \in \operatorname{Stab}(\mathcal{P})$ is then just $a_{0} \cdots a_{n-1}$.

Lemma 9.3. Let $\rho$ and $\sigma$ be (not necessarily distinct) binary relations on $\{0,1, \ldots$, $n-1\}$ such that $\rho$ and $\sigma^{-1}$ are total but $\rho, \sigma \notin \operatorname{Sym}(n)$. Then the semigroup $\langle\operatorname{Sym}(n), \rho, \sigma\rangle$ contains the total relation $n \times n$.

Proof. We prove that there exists $\tau_{0} \in\langle\operatorname{Sym}(n), \rho, \sigma\rangle$ such that $0 \tau_{0}=\{0,1, \ldots$, $n-1\}$. If this is the case, then by replacing $\rho$ by $\sigma^{-1}$ and $\sigma$ by $\rho^{-1}$, there exists $\tau_{1} \in$ $\left\langle\operatorname{Sym}(n), \sigma^{-1}, \rho^{-1}\right\rangle$ such that $0 \tau_{1}=\{0,1, \ldots, n-1\}$. Hence $\tau_{1}^{-1} \in\langle\operatorname{Sym}(n), \rho, \sigma\rangle$ and $\tau_{1}^{-1} \tau_{0}=n \times n$, as required.

We may assume without loss of generality that $0 \rho=\{i:(0, i) \in \rho\} \neq\{0,1, \ldots$, $n-1\}$. Let $A$ be a subset of $\{0,1, \ldots, n-1\}$ with least cardinality such that

$$
A \sigma=\{j:(\exists i \in A)(i, j) \in \sigma\}=\{0,1, \ldots, n-1\} .
$$

Since $\sigma \notin \operatorname{Sym}(n)$, it follows that $|A|<n$ and without loss of generality that $0 \in A$ and $|0 \sigma|>1$. Also by the minimality of $A$, for all $i \in A$ there exists $j \in i \sigma$ such that $j \notin(A \backslash\{i\}) \sigma$.

If $|0 \rho| \geq|A|$, then let $a_{0} \in \operatorname{Sym}(n)$ be any permutation such that $A \subseteq 0 \rho a_{0}$. In this case, $0 \rho a_{0} \sigma=\{0,1, \ldots, n-1\}$, as required. If $|0 \rho|<|A|$, then let $a_{0} \in \operatorname{Sym}(n)$ be any permutation such that $0 \in 0 \rho a_{0}$ and $0 \rho a_{0} \subsetneq A$. In this case, $\left|0 \rho a_{0} \sigma\right| \geq$ $|0 \rho|+1>|0 \rho|$. By repeating this argument we find $a_{1}, a_{2}, \ldots, a_{m} \in \operatorname{Sym}(n)$ such that $0 \rho a_{0} \sigma a_{1} \sigma \cdots a_{m} \sigma=\{0,1, \ldots, n-1\}$, as required.

Lemma 9.4. Let $f \in \Omega^{\Omega}$ be injective such that $d(f)>0$. Then there exists an injective $f^{*} \in\langle\operatorname{Stab}(\mathcal{P}), f\rangle$ such that $\left|\Sigma_{i} \backslash \Omega f^{*}\right| \geq d(f)$ for all $i$ with $0 \leq i \leq n-1$. If $d(f)$ is infinite, then $\left|\Sigma_{i} \backslash \Omega f^{*}\right|=d(f)$ for all $i$.

Proof. Let $\mu=d(f)$ and let $g=f^{2 n}$. By Lemma 5.4(iii) and (iv), $g$ is injective and $d(g)=2 n \mu$. In particular, there exists $0 \leq i \leq n-1$ such that $\left|\Sigma_{i} \backslash \Omega g\right| \geq 2 \mu$. If $\left|\Sigma_{j} \backslash \Omega g\right| \geq \mu$ for all $0 \leq j \leq n-1$, then the proof is completed by setting $f^{*}=g$. Suppose that there exists $j$ such that $0 \leq j \leq n-1$ and $\left|\Sigma_{j} \backslash \Omega g\right|<\mu$. It follows that $j \rho_{g}^{-1} \neq \emptyset$, and so there exists $a \in \operatorname{Stab}(\mathcal{P})$ such that $i \rho_{a} \subseteq j \rho_{g}^{-1}$ and $\left|\left(\Sigma_{i} \backslash \Omega g\right) a \cap \Sigma_{j} g^{-1}\right| \geq \mu$. Hence

$$
\left(\Sigma_{i} \backslash \Omega g\right) a g \cap \Sigma_{j} \subseteq(\Omega \backslash \Omega g) a g \cap \Sigma_{j} \subseteq(\Omega \backslash \Omega g a g) \cap \Sigma_{j}=\Sigma_{j} \backslash \Omega g a g
$$

and so

$\left|\Sigma_{j} \backslash \Omega g a g\right| \geq\left|\left(\Sigma_{i} \backslash \Omega g\right) a g \cap \Sigma_{j}\right| \geq\left|\left(\Sigma_{i} \backslash \Omega g\right) a g \cap \Sigma_{j} g^{-1} g\right|=\left|\left(\Sigma_{i} \backslash \Omega g\right) a \cap \Sigma_{j} g^{-1}\right| \geq \mu ;$ see Figure 10. Also, for all $0 \leq k \leq n-1$ such that $\left|\Sigma_{k} \backslash \Omega g\right| \geq \mu$, we have $\left|\Sigma_{k} \backslash \Omega g a g\right| \geq \mu$. Thus, by repeating this process at most $n-1$ times, we obtain the required $f^{*}$.

If $d(f)$ is infinite and $h \in\langle\operatorname{Stab}(\mathcal{P}), f\rangle$, then either $d(h)=0$ or $d(h)=d(f)$ by Lemma 5.4(ii) and (iii). The final statement follows immediately. 


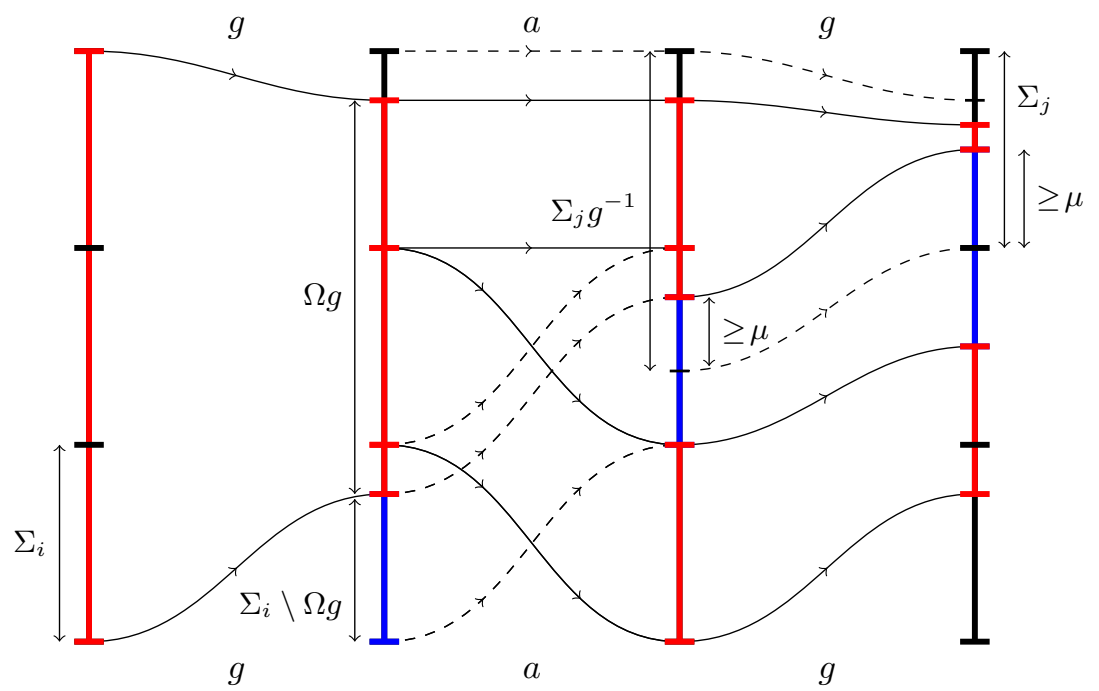

Figure 10. The composite gag in the proof of Lemma 9.4

Lemma 9.5. Let $g \in \Omega^{\Omega}$ be surjective such that $c(g)>0$. Then there exists $g^{*} \in\langle\operatorname{Stab}(\mathcal{P}), g\rangle$ and a transversal $\Gamma$ of $g^{*}$ such that $\left|\Sigma_{i} \backslash \Gamma\right| \geq c(g)$ for all $i$ with $0 \leq i \leq n-1$. If $c(g)$ is infinite, then $\left|\Sigma_{i} \backslash \Gamma\right|=c(g)$ for all $i$.

Proof. If $f$ is any inverse of $g$, then $f$ is injective and $d(f)=c(g)>0$, and so by Lemma 9.4 there exists $f^{*} \in\langle\operatorname{Stab}(\mathcal{P}), f\rangle$ such that $\left|\Sigma_{i} \backslash \Omega f^{*}\right| \geq d(f)$ for all $i$ with $0 \leq i \leq n-1$. But every element of $\langle\operatorname{Stab}(\mathcal{P}), f\rangle$ is injective, and so $\Omega$ is the unique transversal of every element in $\langle\operatorname{Stab}(\mathcal{P}), f\rangle$. In particular, if $\Lambda$ is the unique assignment of transversals for $\langle\operatorname{Stab}(\mathcal{P}), f\rangle$, then $\langle\operatorname{Stab}(\mathcal{P}), f\rangle=\mathcal{C}(\langle\operatorname{Stab}(\mathcal{P}), f\rangle, \Lambda)$ and so $f^{*} \in \mathcal{C}(\langle\operatorname{Stab}(\mathcal{P}), f\rangle, \Lambda)$. Thus, by Corollary [5.3, $f^{*}$ has an inverse $g^{*}$ in $\langle\operatorname{Stab}(\mathcal{P}), g\rangle$. Moreover, if $\Gamma=\Omega f^{*}$, then $\Gamma$ is a transversal of $g^{*}$ and

$$
\left|\Sigma_{i} \backslash \Gamma\right|=\left|\Sigma_{i} \backslash \Omega f^{*}\right| \geq d(f)=c(g)
$$

for all $i$.

Lemma 9.6. Let $U$ be a subsemigroup of $\Omega^{\Omega}$ containing $\operatorname{Stab}(\mathcal{P})$ such that there exist $f, g, t \in U$ and the following hold:

(i) $f$ is injective, $g$ is surjective, and $d(f)=c(g)=|\Omega|$;

(ii) $\rho_{t}=n \times n$.

Then $\operatorname{Sym}(\Omega)$ is contained in $U$.

Proof. We start by showing that there are $f_{0}, g_{0} \in U$ such that $f_{0}$ is injective, $\Omega f_{0}$ is a moiety of $\Sigma_{0}$, and $\Omega f_{0} g_{0}=\Omega$. By Lemma 9.4 there exists $f^{*} \in\langle\operatorname{Stab}(\mathcal{P}), f\rangle$ such that $\left|\Sigma_{i} \backslash \Omega f^{*}\right|=|\Omega|$ for all $i$ such that $0 \leq i \leq n-1$. Since $f$ is injective, every element of $\langle\operatorname{Stab}(\mathcal{P}), f\rangle$ is injective, and so, in particular, $f^{*}$ is injective. Let $0 \leq i \leq n-1$ be arbitrary. By assumption, $\Omega f^{*} \cap \Sigma_{i}$ is contained in a moiety of $\Sigma_{i}$. Also since $\rho_{t}=n \times n$, it follows that $\Sigma_{0} t^{-1} \cap \Sigma_{i}$ is a moiety of $\Sigma_{i}$. In particular, there exists a transversal $\Gamma_{i}$ of $\left.t\right|_{\Sigma_{0} t^{-1} \cap \Sigma_{i}}$ such that $\Gamma_{i}$ is a moiety of $\Sigma_{i}$. Hence there exists $a_{0} \in \operatorname{Stab}(\mathcal{P})$ such that $\left(\Omega f^{*} \cap \Sigma_{i}\right) a_{0} \subseteq \Gamma_{i}$ for all $i$. Then $\Omega f^{*} a_{0} t \subseteq \Sigma_{0}$ and so $\Omega\left(f^{*} a_{0} t\right)^{2}$ is a moiety of $\Sigma_{0}$. Thus $f_{0}=\left(f^{*} a_{0} t\right)^{2}$ is the required mapping. 
For each $i$, let $\Delta_{i}$ be a transversal of $\left.t\right|_{\Sigma_{i} t^{-1} \cap \Sigma_{0}}: \Sigma_{0} \rightarrow \Sigma_{i}$. So each $\Delta_{i}$ is a moiety of $\Sigma_{0}$. Let $a_{1} \in \operatorname{Stab}(\mathcal{P})$ be any permutation such that $\Omega f_{0} a_{1} \cap \Delta_{i}$ is a moiety of $\Delta_{i}$ for all $i$. Then $\left|\Omega f_{0} a_{1} t \cap \Sigma_{i}\right|=|\Omega|$ for all $i$. By Lemma 9.5, there exist $g^{*} \in\langle\operatorname{Stab}(\mathcal{P}), g\rangle$ and a transversal $\Lambda$ of $g^{*}$ such that $\left|\Sigma_{i} \backslash \Lambda\right|=|\Omega|$ for all $i$ such that $0 \leq i \leq n-1$. In other words, $\Lambda \cap \Sigma_{i}$ is contained in a moiety of $\Sigma_{i}$ for all $i$. Since $g$ is surjective, every element of $\langle\operatorname{Stab}(\mathcal{P}), g\rangle$ is surjective, and so $g^{*}$ is surjective. Therefore there exists $a_{2} \in \operatorname{Stab}(\mathcal{P})$ such that $\Omega f_{0} a_{1} t a_{2}$ contains $\Lambda$. Hence $\Omega f_{0} a_{1} t a_{2} g^{*}=\Omega g^{*}=\Omega$ and $g_{0}=a_{1} t a_{2} g^{*}$ is the required function.

To conclude, let $b \in \operatorname{Sym}(\Omega)$ be arbitrary. Then if $\Gamma$ is a transversal of $g_{0}$ contained in $\Omega f_{0}$, there exists $a_{3} \in \operatorname{Stab}(\mathcal{P})$ such that $\alpha f_{0} a_{3} \in \alpha b g_{0}^{-1} \cap \Gamma$ for all $\alpha \in \Omega$. But then $b=f_{0} a_{3} g_{0}$, and so $\operatorname{Sym}(\Omega)$ is contained in $U$, as required.

At this stage it is straightforward to classify the maximal subsemigroups of $\Omega^{\Omega}$ containing the almost stabiliser of a finite partition using the results proved so far. Since the stabiliser is a subgroup of the almost stabiliser, this classification is actually a corollary of Theorem $\mathrm{D}$. To prove the more general Theorem $\mathrm{D}$ we require two further lemmas which are similar to Lemmas 6.3 and 6.4

Corollary 9.7. Let $\Omega$ be any infinite set and let $\mathcal{P}=\left\{\Sigma_{0}, \Sigma_{1}, \ldots, \Sigma_{n-1}\right\}, n \geq 2$, be a finite partition of $\Omega$. Then the maximal subsemigroups of $\Omega^{\Omega}$ containing $\operatorname{AStab}(\mathcal{P})$ but not $\operatorname{Sym}(\Omega)$ are:

$$
\begin{aligned}
& A_{1}(\mathcal{P})=\left\{f \in \Omega^{\Omega}: \rho_{f} \in \operatorname{Sym}(n) \text { or } \rho_{f} \text { is not total }\right\} \\
& A_{2}(\mathcal{P})=\left\{f \in \Omega^{\Omega}: \rho_{f} \in \operatorname{Sym}(n) \text { or } \rho_{f}^{-1} \text { is not total }\right\} .
\end{aligned}
$$

Proof. Let $M$ be a subsemigroup of $\Omega^{\Omega}$ containing $\operatorname{AStab}(\mathcal{P})$ but which is not contained in any of the semigroups listed in Theorems $\mathrm{A}$ or D As in the proof of Theorem B, it suffices to show that $M=\Omega^{\Omega}$.

Since $M \nsubseteq A_{1}(\mathcal{P}), A_{2}(\mathcal{P})$, there exist $f, g \in M$ such that $\rho_{f}$ and $\rho_{g}^{-1}$ are total but $\rho_{f}, \rho_{g} \notin \operatorname{Sym}(n)$. Hence, by Lemmas 9.2 and 9.3 , there exists $t \in M$ such that $\rho_{t}=n \times n$. Since $\operatorname{AStab}(\mathcal{P})$ contains $\{a \in \operatorname{Sym}(\Omega):|\operatorname{supp}(a)|<|\Omega|\}$, it follows by Lemmas 6.3 and 6.4 that there exist $f^{*}, g^{*} \in M$ such that $f^{*}$ is injective, $g^{*}$ is surjective, and $d\left(f^{*}\right)=c\left(g^{*}\right)=|\Omega|$. Thus, by Lemma 9.6. $\operatorname{Sym}(\Omega)$ is contained in $M$, and so, by Theorem $\underline{\mathrm{A}}, M=\Omega^{\Omega}$, as required.

We return to the proof of Theorem D,

Lemma 9.8. Let $U$ be a subset of $\Omega^{\Omega}$ which contains $\operatorname{Stab}(\mathcal{P})$ but which is not contained in $S_{2}$ or $S_{4}(\mu)$ for any infinite $\mu \leq|\Omega|$, and let $\Lambda$ be any assignment of transversals for $U$ (as defined in Definition [5.2). Then there exists an injective $f \in \mathcal{C}(U, \Lambda)$ such that $d(f)=|\Omega|$.

Proof. We prove by transfinite induction that for all cardinals $\mu \leq|\Omega|$,

$$
\text { there exists an injective } f_{\mu} \in \mathcal{C}(U, \Lambda) \text { with } d\left(f_{\mu}\right) \geq \mu \text {. }
$$

Since $U$ is not contained in $S_{2}$, there exists an injective $h_{0} \in U \subseteq \mathcal{C}(U, \Lambda)$ such that $d\left(h_{0}\right)>0$. By taking powers of $h_{0}$ (which also belong to $\mathcal{C}(U, \Lambda)$ ) and applying Lemma 5.4(iii) and (iv), it follows that (5) holds for all finite $\mu$.

Let $\mu$ be any cardinal such that $\aleph_{0} \leq \mu \leq|\Omega|$ and assume that (5) holds for every cardinal $\nu<\mu$. Since $U \nsubseteq S_{4}(\mu)$, there exists $h_{1} \in U$ such that $c\left(h_{1}\right)<\mu \leq d\left(h_{1}\right)$. By our inductive hypothesis, there exists an injective $f_{c\left(h_{1}\right)} \in \mathcal{C}(U, \Lambda)$ such that $d\left(f_{c\left(h_{1}\right)}\right) \geq c\left(h_{1}\right)$. 


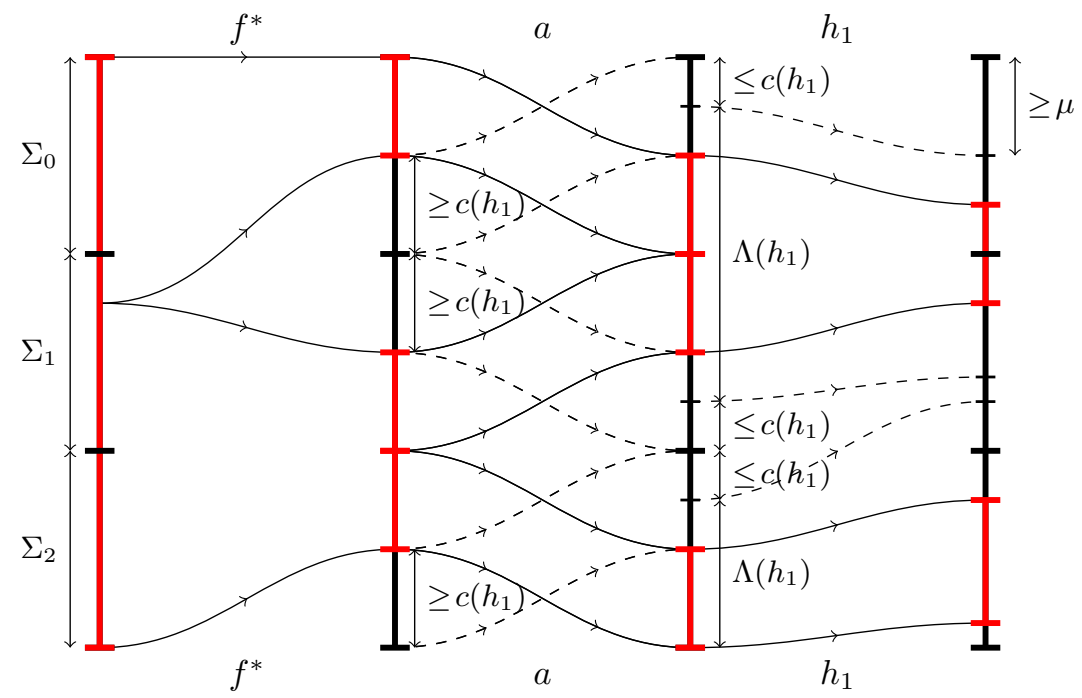

Figure 11. The composite $f^{*} a h_{1}$ in the proof of Lemma 9.8

By Lemma 9.4 there exists an injective $f^{*} \in\left\langle\operatorname{Stab}(\mathcal{P}), f_{c\left(h_{1}\right)}\right\rangle$ such that $\left|\Sigma_{i} \backslash \Omega f^{*}\right| \geq d\left(f_{c\left(h_{1}\right)}\right)$ for all $0 \leq i \leq n-1$. Since every element of $\left\langle\operatorname{Stab}(\mathcal{P}), f_{c\left(h_{1}\right)}\right\rangle$ is injective, it follows that $\left\langle\operatorname{Stab}(\mathcal{P}), f_{c\left(h_{1}\right)}\right\rangle \subseteq \mathcal{C}(U, \Lambda)$ and so $f^{*} \in \mathcal{C}(U, \Lambda)$. Then, since

$$
\left|\Sigma_{i} \backslash \Lambda\left(h_{1}\right)\right| \leq\left|\Omega \backslash \Lambda\left(h_{1}\right)\right|=c\left(h_{1}\right) \leq d\left(f_{c\left(h_{1}\right)}\right) \leq\left|\Sigma_{i} \backslash \Omega f^{*}\right|
$$

for all $0 \leq i \leq n-1$, there exists $a \in \operatorname{Stab}(\mathcal{P})$ such that $\left(\Omega f^{*} \cap \Sigma_{i}\right) a \subseteq \Lambda\left(h_{1}\right) \cap \Sigma_{i}$ for all $i$; see Figure 11. Hence, if we set $f_{\mu}=f^{*} a h_{1}$, then, since $f_{\mu}, a \in \mathcal{C}(U, \Lambda)$ and by the definition of $a$, it follows that $f_{\mu} \in \mathcal{C}(U, \Lambda), f_{\mu}$ is injective, and $d\left(f_{\mu}\right) \geq$ $d\left(h_{1}\right) \geq \mu$ by Lemma 5.4(ii), as required.

Lemma 9.9. Let $U$ be a subset of $\Omega^{\Omega}$ which contains $\operatorname{Stab}(\mathcal{P})$ but which is not contained in $S_{1}$ or $S_{3}(\mu)$ for any infinite $\mu \leq|\Omega|$. Then there exists a surjective $g \in U$ such that $c(g)=|\Omega|$.

Proof. Let $u^{\prime} \in \Omega^{\Omega}$ be an arbitrary inverse for $u$ for all $u \in U$. We denote $\left\{u^{\prime} \in\right.$ $\left.\Omega^{\Omega}: u \in U\right\}$ by $U^{\prime}$ and we set $\Lambda: U^{\prime} \rightarrow \mathcal{P}(\Omega)$ to be the assignment of transversals for $U^{\prime}$ defined by $\Lambda\left(u^{\prime}\right)=\Omega u$. Recall that $c(u)=d\left(u^{\prime}\right)$ and $d(u)=c\left(u^{\prime}\right)$ for all $u \in U$.

We prove that $U^{\prime}$ satisfies the conditions of Lemma 9.4. Since $U \nsubseteq S_{1}, U \nsubseteq$ $S_{3}(\mu)$, it follows that $U^{\prime} \nsubseteq S_{2}$ and $U^{\prime} \nsubseteq S_{4}(\mu)$ for all infinite $\mu \leq|\Omega|$. Thus by Lemma 9.4 there exists an injective $f^{*} \in \mathcal{C}\left(U^{\prime}, \Lambda\right)$ such that $d\left(f^{*}\right)=|\Omega|$. By Corollary 5.3, $\langle U\rangle$ contains an inverse $g^{*}$ of $f^{*}$. Therefore $c\left(g^{*}\right)=d\left(f^{*}\right)=|\Omega|$.

Proof of Theorem D, Let $M$ be a subsemigroup of $\Omega^{\Omega}$ containing $\operatorname{Stab}(\mathcal{P})$ but not contained in any of the semigroups listed in Theorems $\mathrm{A}$ or $\mathrm{D}$. Since $M \nsubseteq$ $A_{1}(\mathcal{P}), A_{2}(\mathcal{P})$, there exists $f, g \in M$ such that $\rho_{f}$ and $\rho_{g}^{-1}$ are total but $\rho_{f}, \rho_{g} \notin$ $\operatorname{Sym}(n)$. Hence, by Lemmas 9.2 and 9.3 , there exists $t \in M$ such that $\rho_{t}=n \times n$. Also by Lemmas 9.8 and 9.9 there exist $f^{*}, g^{*} \in M$ such that $f^{*}$ is injective, $g^{*}$ is 
surjective, and $d\left(f^{*}\right)=c\left(g^{*}\right)=|\Omega|$. Thus, by Lemma 9.6. $\operatorname{Sym}(\Omega)$ is contained in $M$, and so, by Theorem $\mathrm{A}, M=\Omega^{\Omega}$, as required.

\section{Maximal Subsemigroups of the Symmetric Group}

In this section we prove that the stabiliser of a non-empty finite set, the almost stabiliser of a finite partition, and the stabiliser of an ultrafilter are maximal subsemigroups of the symmetric group and not just maximal subgroups.

Let $T$ be a subsemigroup of $\operatorname{Sym}(\Omega)$, and let $G$ denote the group generated by $T$. If $G \neq \operatorname{Sym}(\Omega)$ and $T \neq G$, then, for any $f \in G \backslash T$, the semigroup generated by $T$ and $f$ is a subsemigroup of $G$. In particular, $\langle T, f\rangle \neq \operatorname{Sym}(\Omega)$ and hence $T$ is not maximal. (We remind the reader that $\langle U\rangle$ always denotes the semigroup generated by $U$.) Hence the group generated by any maximal subsemigroup of $\operatorname{Sym}(\Omega)$ that is not a subgroup is $\operatorname{Sym}(\Omega)$.

Theorem 10.1. Let $\Omega$ be any infinite set and let $\Sigma$ be a non-empty finite subset of $\Omega$. Then the setwise stabiliser $\operatorname{Sym}(\Omega)_{\{\Sigma\}}$ of $\Sigma$ in $\operatorname{Sym}(\Omega)$ is a maximal subsemigroup of $\operatorname{Sym}(\Omega)$.

Proof. Let $f \in \operatorname{Sym}(\Omega) \backslash \operatorname{Sym}(\Omega)_{\{\Sigma\}}$. We must show that $\left\langle\operatorname{Sym}(\Omega)_{\{\Sigma\}}, f\right\rangle=$ $\operatorname{Sym}(\Omega)$, i.e. that the semigroup generated by $\operatorname{Sym}(\Omega)_{\{\Sigma\}}$ and $f$ is $\operatorname{Sym}(\Omega)$. Since $\operatorname{Sym}(\Omega)_{\{\Sigma\}}$ is a maximal subgroup of $\operatorname{Sym}(\Omega)$, it suffices to find $g \in\left\langle\operatorname{Sym}(\Omega)_{\{\Sigma\}}, f\right\rangle$ such that $g$ has finite order and $g \notin \operatorname{Sym}(\Omega)_{\{\Sigma\}}$. By postmultiplying by an element of $\operatorname{Sym}(\Omega)_{\{\Sigma\}}$ if necessary, we may assume without loss of generality that every nontrivial cycle of $f$ contains an element of $\Sigma$. Since $\Sigma$ is finite, if every cycle of $f$ is finite, then $f$ itself has finite order, and setting $g=f$ concludes the proof in this case. So suppose $f$ has at least one infinite cycle. There exists $m \in \mathbb{N}$ such that $f^{m}$ has only infinite cycles, each of which contains at most one element of $\Sigma$. Again we may assume without loss of generality that every nontrivial cycle of $f^{m}$ contains precisely one element of $\Sigma$. Then $f^{m}=c_{1} \cdots c_{r}$, where $c_{1}, \ldots, c_{r}$ are disjoint infinite cycles. We may write $c_{i}=\left(\ldots, \alpha_{i,-1}, \alpha_{i, 0}, \alpha_{i, 1}, \alpha_{i, 2}, \ldots\right)$ where $\alpha_{i, 0} \in \Sigma$. We let $d_{i}=\left(\ldots, \alpha_{i, 2}, \alpha_{i, 1}, \alpha_{i,-1}, \alpha_{i,-2}, \ldots\right)$ and $h=d_{1} \cdots d_{r}$. Then $h \in \operatorname{Sym}(\Omega)_{\{\Sigma\}}$ and

$$
g=h f^{m}=\left(\alpha_{1,0}, \alpha_{1,1}\right) \cdots\left(\alpha_{r, 0}, \alpha_{r, 1}\right) \in\left\langle\operatorname{Sym}(\Omega)_{\{\Sigma\}}, f\right\rangle \backslash \operatorname{Sym}(\Omega)_{\{\Sigma\}}
$$

has order 2, which completes the proof.

If $H$ and $K$ are subgroups of a group $G$, then the subsemigroup generated by $H$ and $K$ equals the group generated by $H$ and $K$. Thus the following two lemmas are immediate consequences of the corresponding results about subgroups given in [9] and [23, Note 3(iii) of $\S 4$ ], respectively.

Lemma 10.2. If $\Gamma_{1}, \Gamma_{2} \subseteq \Omega$ and $\left|\Gamma_{1} \cap \Gamma_{2}\right|=\min \left\{\left|\Gamma_{1}\right|,\left|\Gamma_{2}\right|\right\}$, then $\operatorname{Sym}\left(\Gamma_{1} \cup \Gamma_{2}\right)$ equals the subsemigroup $\left\langle\operatorname{Sym}\left(\Gamma_{1}\right), \operatorname{Sym}\left(\Gamma_{2}\right)\right\rangle$ generated by the subgroups $\operatorname{Sym}\left(\Gamma_{1}\right)$ and $\operatorname{Sym}\left(\Gamma_{2}\right)$.

Lemma 10.3. Let $S$ be a subsemigroup of $\operatorname{Sym}(\Omega)$. If $S$ contains $\operatorname{Sym}(\Sigma)$ for all moieties $\Sigma$ of $\Omega$, then $S=\operatorname{Sym}(\Omega)$.

Lemma 10.4. Let $S$ be a subsemigroup of $\operatorname{Sym}(\Omega)$. If $S$ contains $\operatorname{Sym}(\Sigma)$ for some moiety $\Sigma$ of $\Omega$ and $S$ is transitive on moieties of $\Omega$, then $S=\operatorname{Sym}(\Omega)$. 
Proof. It suffices by Lemma 10.3 to show that $S$ contains $\operatorname{Sym}(\Gamma)$ for every moiety $\Gamma$ of $\Omega$. Let $\Gamma$ be any moiety of $\Omega$ and let $f \in \operatorname{Sym}(\Gamma)$ be arbitrary. There exist $g, h, k \in S$ such that

$$
\Gamma g=\Sigma, \quad \Sigma h=\Omega \backslash \Sigma, \quad(\Omega \backslash \Sigma) k=\Gamma .
$$

Since $(\Sigma) g^{-1} f k^{-1} h^{-1}=\Sigma$, it follows that there exists $a \in \operatorname{Sym}(\Sigma) \subseteq S$ such that $\left.a\right|_{\Sigma}=\left.g^{-1} f k^{-1} h^{-1}\right|_{\Sigma}$. Also $\Sigma h^{-1} g^{-1} k^{-1}=\Sigma$, there exists $b \in \operatorname{Sym}(\Sigma) \subseteq S$ such that $\left.b\right|_{\Sigma}=\left.h^{-1} g^{-1} k^{-1}\right|_{\Sigma}$.

We will show that $f=g a h b k \in S$. If $\alpha \in \Omega \backslash \Gamma$ is arbitrary, then $\alpha g \in \Omega \backslash \Sigma$ and so $\alpha g a=\alpha g$, and $\alpha g h \in \Sigma$ and so $\alpha g h b=\alpha k^{-1}$. Therefore

$$
(\alpha) g a h b k=(\alpha) g h b k=(\alpha) k^{-1} k=\alpha .
$$

If $\beta \in \Gamma$, then $\beta g \in \Sigma$ and so $\beta g a=\beta f k^{-1} h^{-1}$. Thus

$$
(\beta) g a h b k=(\beta) f k^{-1} b k,
$$

and since $\beta f k^{-1} \in \Omega \backslash \Sigma$ and $b$ fixes $\Omega \backslash \Sigma$ pointwise, it follows that

$$
(\beta) g a h b k=(\beta) f,
$$

as required.

Let $\Omega$ be an infinite set, let $\mathcal{P}=\left\{\Sigma_{0}, \Sigma_{1}, \ldots, \Sigma_{n-1}\right\}, n \geq 2$, be a finite partition of $\Omega$, and let $f \in \Omega^{\Omega}$. Recall that the binary relation $\rho_{f}$ on $\{0,1, \ldots, n-1\}$ is defined in Equation (11) in Section 2.4 as

$$
\rho_{f}=\left\{(i, j):\left|\Sigma_{i} f \cap \Sigma_{j}\right|=|\Omega|\right\} .
$$

Theorem 10.5. Let $\Omega$ be any infinite set and let $\mathcal{P}=\left\{\Sigma_{0}, \Sigma_{1}, \ldots, \Sigma_{n-1}\right\}, n \geq 2$, be a finite partition of $\Omega$. Then $\operatorname{AStab}(\mathcal{P})$ is a maximal subsemigroup of $\operatorname{Sym}(\Omega)$.

Proof. Let $f \in \operatorname{Sym}(\Omega) \backslash \operatorname{AStab}(\mathcal{P})$ be arbitrary. Then by Lemmas 9.2 and 9.3 there exists $g \in\langle\operatorname{AStab}(\mathcal{P}), f\rangle$ such that $\rho_{g}=n \times n$.

Let $h \in \operatorname{Sym}\left(\Sigma_{0} g^{-1}\right)$. We will show that $h=g b g a$ for some $a, b \in \operatorname{AStab}(\mathcal{P})$. (In fact, $a, b$ will belong to $\operatorname{Stab}(\mathcal{P})$.)

Since $\rho_{g}=n \times n$, both $\Sigma_{i} g^{-1} \cap \Sigma_{j}$ and $\Sigma_{i} g \cap \Sigma_{j}$ are moieties in $\Sigma_{j}$ for all $i, j \in\{0,1, \ldots, n-1\}$. It follows that there exists $a \in \operatorname{AStab}(\mathcal{P})$ such that

$$
\left(\Sigma_{i} g\right) a=\Sigma_{i} g^{-1}
$$

for all $i$. Define $b \in \operatorname{Sym}(\Omega)$ by $\alpha b=\alpha g^{-1} h a^{-1} g^{-1}$ if $\alpha \in \Sigma_{0}$ and $\alpha b=\alpha g^{-1} a^{-1} g^{-1}$ if $\alpha \notin \Sigma_{0}$. Since $h \in \operatorname{Sym}\left(\Sigma_{0} g^{-1}\right)$, it follows that $\left(\Sigma_{0} g^{-1}\right) h=\Sigma_{0} g^{-1}$ and so

$$
\Sigma_{0} b=\Sigma_{0} g^{-1} h a^{-1} g^{-1}=\Sigma_{0} g^{-1} a^{-1} g^{-1}=\Sigma_{0} g g^{-1}=\Sigma_{0},
$$

and if $i \neq 0$, then

$$
\Sigma_{i} b=\Sigma_{i} g^{-1} a^{-1} g^{-1}=\Sigma_{i} g g^{-1}=\Sigma_{i} .
$$

Hence $b \in \operatorname{AStab}(\mathcal{P})$. Let $\alpha \in \Omega$ be arbitrary. If $\alpha \in \Sigma_{0} g^{-1}$, then $\alpha g \in \Sigma_{0}$, and so

$$
\alpha g b g a=\alpha g g^{-1} h a^{-1} g^{-1} g a=\alpha h .
$$

If $\alpha \notin \Sigma_{0} g^{-1}$, then

$$
\alpha g b g a=\alpha g g^{-1} a^{-1} g^{-1} g a=\alpha=\alpha h,
$$

and so $h=g b g a$, as required. It follows that $\operatorname{Sym}\left(\Sigma_{0} g^{-1}\right) \leq\langle\operatorname{AStab}(\mathcal{P}), f\rangle$. Therefore, since $\Sigma_{0} g^{-1} \cap \Sigma_{0}$ and $\Sigma_{0} g^{-1} \cap \Sigma_{1}$ are moieties in $\Sigma_{0}$ and $\Sigma_{1}$, respectively, and by Lemma 10.2 .

$$
\operatorname{Sym}\left(\Sigma_{0} \cup \Sigma_{1}\right) \leq\left\langle\operatorname{Sym}\left(\Sigma_{0}\right), \operatorname{Sym}\left(\Sigma_{1}\right), \operatorname{Sym}\left(\Sigma_{0} g^{-1}\right)\right\rangle \leq\langle\operatorname{AStab}(\mathcal{P}), f\rangle .
$$


Since $\operatorname{AStab}(\mathcal{P})$ is 2-transitive on $\Sigma_{0}, \ldots, \Sigma_{n-1}$, we conclude that $\langle\operatorname{AStab}(\mathcal{P}), f\rangle=$ $\operatorname{Sym}(\Omega)$, and so $\operatorname{AStab}(\mathcal{P})$ is a maximal subsemigroup of $\operatorname{Sym}(\Omega)$.

Theorem 10.6. Let $\mathcal{F}$ be an ultrafilter on $\Omega$. Then the stabiliser $\operatorname{Sym}(\Omega)_{\{\mathcal{F}\}}$ of $\mathcal{F}$ is a maximal subsemigroup of $\operatorname{Sym}(\Omega)$.

Proof. Let $f \in \operatorname{Sym}(\Omega) \backslash \operatorname{Sym}(\Omega)_{\{\mathcal{F}\}}$. Then either:

(i) there is a subset $\Sigma$ of $\Omega$ such that $\Sigma \in \mathcal{F}$ and $\Sigma f \notin \mathcal{F}$, or

(ii) there is a subset $\Gamma$ of $\Omega$ such that $\Gamma \notin \mathcal{F}$ and $\Gamma f \in \mathcal{F}$.

It is straightforward to verify that $\Sigma$ and $\Gamma$ can be chosen to be moieties of $\Omega$. If (i) holds, then (ii) holds with $\Gamma=\Omega \backslash \Sigma$. If (ii) holds, then (i) holds with $\Sigma=\Omega \backslash \Gamma$. So we may assume that both (i) and (ii) hold. Let $\Lambda$ and $\Delta$ be moieties of $\Omega$. If $\Lambda$ and $\Delta$ both belong to $\mathcal{F}$ or neither belongs to $\mathcal{F}$, then there exists $a_{0} \in \operatorname{Sym}(\Omega)_{\{\mathcal{F}\}}$ such that $\Lambda a_{0}=\Delta$. If $\Lambda \in \mathcal{F}$ and $\Delta \notin \mathcal{F}$, then we choose $a_{1}, a_{2} \in \operatorname{Sym}(\Omega)_{\{\mathcal{F}\}}$ such that $\Lambda a_{1}=\Sigma$ and $(\Sigma f) a_{2}=\Delta$, and note that $\Lambda a_{1} f a_{2}=\Delta$. Similarly, if $\Lambda \notin \mathcal{F}$ and $\Delta \in \mathcal{F}$, then there exists $a_{3}, a_{4} \in \operatorname{Sym}(\Omega)_{\{\mathcal{F}\}}$ such that $\Lambda a_{3} f a_{4}=\Delta$. We have shown that $\left\langle\operatorname{Sym}(\Omega)_{\{\mathcal{F}\}}, f\right\rangle$ is transitive on moieties. Since $\operatorname{Sym}(\Omega)_{\{\mathcal{F}\}}$ is full on every moiety $\Xi \notin \mathcal{F}$, the result follows from Lemma 10.4 .

\section{REFERENCES}

[1] M. Aschbacher and L. Scott, Maximal subgroups of finite groups, J. Algebra 92 (1985), no. 1, 44-80, DOI 10.1016/0021-8693(85)90145-0. MR772471 (86m:20029)

[2] Ralph W. Ball, Maximal subgroups of symmetric groups, Trans. Amer. Math. Soc. 121 (1966), 393-407. MR0202813 (34 \#2672)

[3] Ralph W. Ball, Indices of maximal subgroups of infinite symmetric groups, Proc. Amer. Math. Soc. 19 (1968), 948-950. MR0229707 (37 \#5281)

[4] James E. Baumgartner, Saharon Shelah, and Simon Thomas, Maximal subgroups of infinite symmetric groups, Notre Dame J. Formal Logic 34 (1993), no. 1, 1-11, DOI 10.1305/ndjfl/1093634559. MR1213842 (94g:20005)

[5] George M. Bergman and Saharon Shelah, Closed subgroups of the infinite symmetric group, Algebra Universalis 55 (2006), no. 2-3, 137-173, DOI 10.1007/s00012-006-1959-z. Special issue dedicated to Walter Taylor. MR2280223 (2008a:20005)

[6] Peter Biryukov and Valery Mishkin, Set ideals with maximal symmetry group and minimal dynamical systems, Bull. London Math. Soc. 32 (2000), no. 1, 39-46, DOI 10.1112/S0024609399006438. MR.1718749 (2000i:20002)

[7] Marcus Brazil, Jacinta Covington, Tim Penttila, Cheryl E. Praeger, and Alan R. Woods, Maximal subgroups of infinite symmetric groups, Proc. London Math. Soc. (3) 68 (1994), no. 1, 77-111, DOI 10.1112/plms/s3-68.1.77. MR.1243836 (94m:20011)

[8] Jacinta Covington, Dugald Macpherson, and Alan Mekler, Some maximal subgroups of infinite symmetric groups, Quart. J. Math. Oxford Ser. (2) 47 (1996), no. 187, 297-311, DOI 10.1093/qmath/47.3.297. MR1412557 (97k:20005)

[9] John D. Dixon, Peter M. Neumann, and Simon Thomas, Subgroups of small index in infinite symmetric groups, Bull. London Math. Soc. 18 (1986), no. 6, 580-586, DOI 10.1112/blms/18.6.580. MR859950 (88i:20004)

[10] R. Dougall, C. Gresens, A. Schmidt-Lademann, and J. Jonušas, Maximal subsemigroups of the maximal subsemigroups of the full transformation semigroup on a countable set, June 2012.

[11] G. P. Gavrilov, On functional completeness in countably-valued logic (Russian), Problemy Kibernet. No. 15 (1965), 5-64. MR0229511 (37 \#5085)

[12] N. Graham, R. Graham, and J. Rhodes, Maximal subsemigroups of finite semigroups, J. Combinatorial Theory 4 (1968), 203-209. MR.0223470 (36 \#6518)

[13] Eckehart Hotzel, Maximality properties of some subsemigroups of Baer-Levi semigroups, Semigroup Forum 51 (1995), no. 2, 153-190, DOI 10.1007/BF02573628. MR1345110 (96m:20096) 
[14] John M. Howie, N. Ruškuc, and P. M. Higgins, On relative ranks of full transformation semigroups, Comm. Algebra 26 (1998), no. 3, 733-748, DOI 10.1080/00927879808826160. MR.1606221 (99b:20099)

[15] Thomas Jech, Set theory, The third millennium edition, revised and expanded, Springer Monographs in Mathematics, Springer-Verlag, Berlin, 2003. MR 1940513 (2004g:03071)

[16] Julius Jonušas and J. D. Mitchell, A finite interval in the subsemigroup lattice of the full transformation monoid, Semigroup Forum 89 (2014), no. 1, 183-198. MR3249877

[17] Inessa Levi and G. R. Wood, On maximal subsemigroups of Baer-Levi semigroups, Semigroup Forum 30 (1984), no. 1, 99-102, DOI 10.1007/BF02573440. MR759699 (86d:20067)

[18] Martin W. Liebeck, Cheryl E. Praeger, and Jan Saxl, On the O'Nan-Scott theorem for finite primitive permutation groups, J. Austral. Math. Soc. Ser. A 44 (1988), no. 3, 389-396. MR.929529(89a:20002)

[19] Dugald Macpherson, Large subgroups of infinite symmetric groups, Finite and infinite combinatorics in sets and logic (Banff, AB, 1991), NATO Adv. Sci. Inst. Ser. C Math. Phys. Sci., vol. 411, Kluwer Acad. Publ., Dordrecht, 1993, pp. 249-278. MR1261210 (94m:20012)

[20] H. D. Macpherson and Peter M. Neumann, Subgroups of infinite symmetric groups, J. London Math. Soc. (2) 42 (1990), no. 1, 64-84, DOI 10.1112/jlms/s2-42.1.64. MR.1078175|(92d:20006)

[21] H. D. Macpherson and Cheryl E. Praeger, Maximal subgroups of infinite symmetric groups, J. London Math. Soc. (2) 42 (1990), no. 1, 85-92, DOI 10.1112/jlms/s2-42.1.85. MR1078176 (92d:20007)

[22] V. Mishkin, Symmetry groups of ideals on $\omega$, Second International Conference on Algebra (Barnaul, 1991), Contemp. Math., vol. 184, Amer. Math. Soc., Providence, RI, 1995, pp. 305316, DOI 10.1090/conm/184/02127. MR1332298 (96c:20006)

[23] Peter M. Neumann, Homogeneity of infinite permutation groups, Bull. London Math. Soc. 20 (1988), no. 4, 305-312, DOI 10.1112/blms/20.4.305. MR940282 (89c:20009)

[24] Michael Pinsker, Maximal clones on uncountable sets that include all permutations, Algebra Universalis 54 (2005), no. 2, 129-148, DOI 10.1007/s00012-005-1929-x. MR.2217632 (2006m:08010)

[25] Michael Pinsker, The number of unary clones containing the permutations on an infinite set, Acta Sci. Math. (Szeged) 71 (2005), no. 3-4, 461-467. MR2206591 (2006j:08005)

[26] Michael Pinsker and Saharon Shelah, Universality of the lattice of transformation monoids, Proc. Amer. Math. Soc. 141 (2013), no. 9, 3005-3011. MR3068953

[27] Fred Richman, Maximal subgroups of infinite symmetric groups, Canad. Math. Bull. 10 (1967), 375-381. MR0214652(35 \#5501)

[28] Leonard L. Scott, Representations in characteristic $p$, The Santa Cruz Conference on Finite Groups (Univ. California, Santa Cruz, Calif., 1979), Proc. Sympos. Pure Math., vol. 37, Amer. Math. Soc., Providence, R.I., 1980, pp. 319-331. MR604599 (82e:20052)

[29] L. B. Shneperman, On maximal compact subsemigroups of the endomorphism semigroup of an $n$-dimensional complex vector space, Semigroup Forum 47 (1993), no. 2, 196-208, DOI 10.1007/BF02573756. MR 1230143 (94g:22002)

Centre for Research in Mathematics, School of Computing, Engineering and Mathematics, University of Western Sydney, Penrith, New South Wales 2751, Australia

Mathematics Institute, University of Saint Andrews, St. Andrews, KY16 9SS, United KINGDOM

Mathematics Institute, University of Saint Andrews, St. Andrews, KY16 9SS, United KINGDOM

Current address: School of Physics, Astronomy and Mathematics, University of Hertfordshire,

Hatfield AL10 9AB, United Kingdom

E-mail address: y.peresse@herts.ac.uk 